\title{
Renormalization group crossover in the critical dynamics of field theories with mode coupling terms
}

\author{
Andrea Cavagna, ${ }^{1,2}$ Luca Di Carlo ${ }^{10},{ }^{2,1, *}$ Irene Giardina, ${ }^{2,1,3}$ Luca Grandinetti, ${ }^{4}$ Tomas S. Grigera, ${ }^{5,6,7}$ and Giulia Pisegna ${ }^{2,1}$ \\ ${ }^{1}$ Istituto Sistemi Complessi, Consiglio Nazionale delle Ricerche, UOS Sapienza, 00185 Rome, Italy \\ ${ }^{2}$ Dipartimento di Fisica, Università Sapienza, 00185 Rome, Italy \\ ${ }^{3}$ INFN, Unità di Roma 1, 00185 Rome, Italy \\ ${ }^{4}$ Dipartimento di Scienza Applicata e Tecnologia, Politecnico di Torino, Torino, Italy \\ ${ }^{5}$ Instituto de Física de Líquidos y Sistemas Biológicos CONICET-Universidad Nacional de La Plata, La Plata, Argentina \\ ${ }^{6}$ CCT CONICET La Plata, Consejo Nacional de Investigaciones Científicas y Técnicas, Argentina \\ ${ }^{7}$ Departamento de Física, Facultad de Ciencias Exactas, Universidad Nacional de La Plata, Argentina
}

(Received 8 May 2019; published 23 December 2019)

\begin{abstract}
Motivated by the collective behavior of biological swarms, we study the critical dynamics of field theories with coupling between order parameter and conjugate momentum in the presence of dissipation. Under a fixednetwork approximation, we perform a dynamical renormalization group calculation at one loop in the nearcritical disordered region, and we show that the violation of momentum conservation generates a crossover between an unstable fixed point, characterized by a dynamic critical exponent $z=d / 2$, and a stable fixed point with $z=2$. Interestingly, the two fixed points have different upper critical dimensions. The interplay between these two fixed points gives rise to a crossover in the critical dynamics of the system, characterized by a crossover exponent $\kappa=4 / d$. The crossover is regulated by a conservation length scale $\mathcal{R}_{0}$, given by the ratio between the transport coefficient and the effective friction, which is larger as the dissipation is smaller: Beyond $\mathcal{R}_{0}$, the stable fixed point dominates, while at shorter distances dynamics is ruled by the unstable fixed point and critical exponent, a behavior which is all the more relevant in finite-size systems with weak dissipation. We run numerical simulations in three dimensions and find a crossover between the exponents $z=3 / 2$ and $z=2$ in the critical slowdown of the system, confirming the renormalization group results. From the biophysical point of view, our calculation indicates that in finite-size biological groups mode coupling terms in the equation of motion can significantly change the dynamical critical exponents even in the presence of dissipation, a step toward reconciling theory with experiments in natural swarms. Moreover, our result provides the scale within which fully conservative Bose-Einstein condensation is a good approximation in systems with weak symmetry-breaking terms violating number conservation, as quantum magnets or photon gases.
\end{abstract}

DOI: 10.1103/PhysRevE.100.062130

\section{INTRODUCTION}

The success of the theory of critical phenomena is based upon a simple observation: Systems with very different microscopic details behave in strikingly similar ways when correlations are sufficiently strong. This experimental fact eventually crossed over into theory with the formulation of the phenomenological scaling laws [1-4], whose key idea is that the only relevant scale ruling the spatiotemporal behavior of a system near its critical point is the correlation length. Eventually, the great conceptual edifice of the renormalization group (RG) tied everything together, explaining why microscopically different systems shared so much at the macroscopic level, giving a demonstration of universality through the concept of attractive fixed points, and providing a

\footnotetext{
*Corresponding author: luca.dicarlo@uniroma1.it
}

Published by the American Physical Society under the terms of the Creative Commons Attribution 4.0 International license. Further distribution of this work must maintain attribution to the author(s) and the published article's title, journal citation, and DOI. method to calculate experimentally accessible quantities, most conspicuously the critical exponents [5-8].

Employing the same set of conceptual tools in collective biological systems could prove very helpful, given the recent massive flow of hugely diverse empirical data that theory has to make sense of. In support of this strategy, there is first an empirical observation regarding collective biological systems, namely systems in which a large numbers of units (cells, bacteria, insects, birds, mammals) interact locally in space and time giving rise to macroscopic patterns $[9,10]$ : These systems often exhibit unusually strong correlations whose spatial range is significantly larger than the microscopic scales [11-15]. Besides, recent experiments on natural swarms found evidence of dynamical scaling, a core mechanism of statistical physics linking spatial correlation to temporal relaxation $[3,16]$, whose validity in a biological context can hardly be considered a coincidence. Hence, despite the temptation, in front of the arresting complexity of biology, to confine ourselves to describing the specifics, we believe that exploring the path correlation-scaling $R G$ is a reasonable course of action $[17,18]$. The hydrodynamic theory of flocking of Toner and Tu has led the way: It applied field-theoretical methods and 
the RG to bird flocks, namely collective biological systems in their strongly ordered phase [19-21]. Here, we use the RG approach to study the other side of collective behavior, namely the near-critical disordered phase of natural swarms.

In the biophysics of collective behavior, a prominent role is played by a class of ferromagnetic theories with continuous symmetries, both in their symmetry-broken phase (flocks) and in the near-critical disordered phase (swarms) [19,22]. When dynamics is taken into consideration, though, this universality class breaks down into smaller subclasses, as there are different ways to implement the dynamics given the same static probability distribution of the system $[23,24]$. Dynamical diversity is regulated essentially by two distinct, though related, factors, namely conservation laws and symmetries. On the one hand, we have dynamical theories lacking symmetries and conservation laws (as in the classic Heisenberg model, or model A of Ref. [23]), or in which conservation is imposed despite the absence of an explicit symmetry (as in phase separation, or model B of Ref. [23]). On the other hand, we have theories ruled by symmetry and conservation laws, whose dynamics is characterized by the coupling between two fields, namely the order parameter and the conserved generator of the symmetry, i.e., the conjugate momentum. This second type of theories therefore has nondissipative mode-coupling terms in the equations of motion and was originally introduced to describe systems displaying Bose-Einstein condensation (BEC), as superfluid helium, superconductivity, and quantum magnets (models E, F, and G of Ref. [23]). Bizarre as it may seem, recent experiments suggest that some collective biological systems, as bird flocks [25] and insect swarms [22], also have nondissipative mode-coupling terms in their dynamical equations and are thus akin to this second class of theories. The connection between BEC systems and flying animals reflects the great generality of the mathematical structure of collective dynamics governed by symmetry and conservation laws, whether the order parameter is the quantum phase of a condensate or the direction of motion of a flock.

Here, we will focus on this second class of theories, with the aim of studying the critical dynamics of swarms. To make this introductory discussion more concrete, let us anticipate the actual dynamical field equations we are going to derive and analyze in detail in this work

$$
\begin{gathered}
\frac{\partial \boldsymbol{\psi}}{\partial t}=-\Gamma_{0} \frac{\delta \mathcal{H}}{\delta \boldsymbol{\psi}}+g_{0} \boldsymbol{\psi} \times \frac{\delta \mathcal{H}}{\delta \boldsymbol{s}}+\boldsymbol{\theta}, \\
\frac{\partial \boldsymbol{s}}{\partial t}=\left(\lambda_{0} \nabla^{2}-\eta_{0}\right) \frac{\delta \mathcal{H}}{\delta \boldsymbol{s}}+g_{0} \boldsymbol{\psi} \times \frac{\delta \mathcal{H}}{\delta \boldsymbol{\psi}}+\zeta,
\end{gathered}
$$

with effective Hamiltonian,

$$
\mathcal{H}=\int d^{d} x\left\{\frac{1}{2}(\nabla \boldsymbol{\psi})^{2}+\frac{1}{2} r_{0} \psi^{2}+u_{0} \psi^{4}+\frac{s^{2}}{2 \chi_{0}}\right\} .
$$

In the biological context, the vector order parameter $\psi(\mathbf{x}, t)$ represents the velocity field, but it has different interpretations in BEC systems (for example, in liquid helium $\psi$ is the expectation value of the Bose field). In all cases, though, the order parameter is coupled to its conjugate momentum, which we call spin, $s(\mathbf{x}, t)$, since it is the generator of rotations of $\psi$, given the rotational symmetry of $\mathcal{H} .{ }^{1}$ The distinctive trait of this class of models is the presence of mode-coupling cross terms, $\partial_{t} \boldsymbol{\psi} \sim \delta_{s} \mathcal{H}$ and $\partial_{t} \boldsymbol{s} \sim \delta_{\psi} \mathcal{H}$, which generate a nondissipative dynamics with the classic coordinate-momentum Hamiltonian structure; were it only for these terms, dynamics would be completely deterministic. On the other hand, the diagonal terms, $\partial_{t} \boldsymbol{\psi} \sim \delta_{\psi} \mathcal{H}$ and $\partial_{t} \boldsymbol{s} \sim \delta_{s} \mathcal{H}$, give rise to the diffusion and transport phenomenology typical of stochastic statistical systems and are thus complemented by the white Gaussian noises $\boldsymbol{\theta}$ and $\boldsymbol{\zeta}$, whose variance is proportional to the kinetic coefficients, $2 \Gamma_{0}$ and $2\left(-\lambda_{0} \nabla^{2}+\eta_{0}\right)$ [see Eq. (17)].

The crucial feature of this theory is that, in absence of dissipation, namely when the effective friction $\eta_{0}$ is zero, the total integral of the spin is conserved: The cross term in (2) gives rise to a continuity equation for the symmetry generator, $\boldsymbol{s}(\mathbf{x}, t)$, prescribed by Noether's theorem, while the stochastic transport term in (2), $\lambda_{0} \nabla^{2} \boldsymbol{s}$, is still the divergence of a current, leaving the continuity equation intact. This structuresymmetry and conservation-is a very profound feature of this class of models, as it leads to the existence of propagating hydrodynamic modes in the ordered phase, called spin waves: This mechanism gives rise to "second sound" in liquid helium [23], is responsible for linear information propagation in bird flocks [27], and explains spin-wave remnants in the nearcritical phase of insect swarms [22].

Why then to introduce in Eq. (2) a dissipative term, $\eta_{0}$, which destroys spin conservation? In the context of biological systems, the answer is quite simple: The symmetry generator, or spin, is conjugated to the velocity field; hence, by rotating the velocity, the spin is what actually makes an animal turn. Indeed, kinematically one can prove that the spin is related to the radius of curvature of the individual trajectories [28]. Hence, at the individual level, it is clear that there must be some dissipation relaxing the spin, thus making a trajectory straight in the absence of external perturbations or interaction with the neighbors. On the other hand, in systems like superfluids or superconductors, the conservation law generated by the continuous symmetry of the quantum phase corresponds to number conservation and it cannot be violated. In other BEC systems, though, like quantum magnets [29], exciton condensates [30], and photon gases [31], the Hamiltonian can contain terms that weakly violate the symmetry, hence dissipating the density in the continuity equation of the momentum. The effect of weak dissipation in the ordered phase is simply to generate a damping length scale on propagating spin waves. However, in the near-critical phase, the situation is more complicated: Dissipative and nondissipative models are known to have completely different critical exponents, and hence the effect of dissipation in this case is unclear. This question is particularly relevant for biological swarms, as experiments found a dynamical critical exponent that cannot

\footnotetext{
${ }^{1}$ More precisely, the field canonically conjugate to $S$ is the phase $\varphi$ of the order parameter $\psi$, not $\psi$ itself; for example, in the planar case the order parameter is a complex field and $\psi=e^{i \varphi}$. This is why cross products enter the dynamical equations; the relationship among order parameter, phase, and spin is similar to that among position, angle, and angular momentum in standard rotational motion-see Ref. [26] for a discussion of this point.
} 
be reconciled with the prediction of purely dissipative theories [22].

Here, by using a dynamical renormalization group approach, we study the effect of dissipation on the critical dynamics of a system with mode coupling terms. Our calculation shows that the dissipative term $\eta_{0}$ gives rise to an interesting crossover characterized by nontrivial critical exponents. At the critical point, where the correlation length is infinite, the RG transformation must leave the system exactly the same, so that the RG fixed points provide the important information on the large-scale properties. In this case, we find two fixed points relevant to the description of the dynamics. The crossover between the two is ruled by the competition between conservative transport, $\lambda_{0} \nabla^{2} s$, and dissipative friction, $-\eta_{0} s$. This generates a novel conservation length scale, $\mathcal{R}_{0}$, beyond which the dynamics is ruled by a purely dissipative RG fixed point, so that the whole conservative (and propagating) nature of the theory is lost. On the other hand, for distances smaller than $\mathcal{R}_{0}$, the conservative RG fixed point governs the dynamics, giving rise to the classic spin-wave phenomenology. We calculate the value of the dynamical critical exponents in these two regimes and of the crossover exponent, and we confirm our results through numerical simulations.

As we shall see, the conservation scale $\mathcal{R}_{0}$ is larger as the dissipation is smaller. The implications of this fact are very important in the biophysical context. The presence of dissipation in the dynamical equations of biological groups may suggest that these systems are in the same universality class as fully dissipative models, since dissipation always wins over conservative terms in the infinite-time and infinitedistance hydrodynamic limit. However, real biological groups are finite-size systems (and quite moderately sized, in the case of flocks and swarms) in which dissipation has been demonstrated by experiments to be quite low [22]. Therefore, the size of these systems may actually be smaller than the crossover scale $\mathcal{R}_{0}$, so that, even if dissipative terms are present in the equations of motion, critical dynamics is still ruled by the symmetric and conservative structure of the equations and therefore displays critical exponents drastically different from the dissipative ones. As we shall see, for natural swarms this theoretical mechanism produces a critical dynamics whose phenomenology is remarkably similar to that found in experiments.

Although our motivation is biological, it is worthwhile to remark that our results apply to any BEC system with weak dissipation. Relevant examples are quantum magnets [29], exciton condensates [30], and photon gases [31]. In quantum magnets, Bose-Einstein condensation of magnons occurs at low temperature, due to the spontaneous breaking of the $U(1)$ symmetry; real quantum magnets, though, contain weakly symmetry-breaking terms in their Hamiltonian, thus violating the conservation of the conjugate momentum. Another BEC system our results could be applied to is that of excitons, bosonic hole-particle excitations created by laser pumps, whose number, though, is conserved only within their lifetime (which is finite and dependent on many factors) [30]. A similar situation arises within the context of photon gases, when a polariton condensate emerges [31]; in this case, too, depending on the polariton lifetime, one can have a violation of number conservation, which is equivalent to an effective dissipation. In all these cases, our calculation could provide the crossover scale within which an exact BEC assumption is justified and it may describe the critical behavior of the crossover.

Here is the plan of the paper. In Sec. II, we will discuss the microscopic model, the inertial spin model, in its biological context. This is introduced as a modified Vicsek model, with the same velocity-aligning interactions but with Hamiltonian dynamics incorporating inertia and conservation of spin (the conjugate momentum). After discussing the fixed-network approximation, we shall then (Sec. III) coarse grain the microscopic ISM equations and work out the dynamical field theory we study, i.e., Eqs. (1) and (2). Our field theory is thus a mesoscopic-level (coarse-grained) description of the ISM under the fixed-network approximation. We then proceed to the renormalization group calculation of the critical dynamics: In Sec. IV, we will perform in the calculation in the momentum shell version of the RG. This section will culminate with the formulation of the $\mathrm{RG}$ recursion equations, while the analysis of the crossover between the two different fixed points on the critical manifold and the corresponding crossover of the critical dynamics will be studied in Sec. V. In Sec. VI, we will give an alternative derivation of our results using the more field-theoretical Callan-Symanzik approach. In Sec. VII, we will perform numerical simulations to validate the $R G$ results, and finally we will present our conclusions and discuss the outlook in Sec. VIII. Parts of the most technical material are contained in the Appendixes. A shorter account of our results can be found in Ref. [32].

\section{BIOPHYSICAL ORIGIN OF THE MICROSCOPIC MODEL}

In this section, we derive the microscopic model of collective behavior that we will use to describe the dynamics of natural swarms. Because this model was first introduced in the context of flocks, rather than swarms, we will have to take a short detour in that direction. At the end of the section, we will discuss under what approximations we will be able to perform a field-theoretical RG study of the model.

\section{A. Collective behavior and the Vicsek model}

Collective behavior in biological systems, and more specifically collective motion, is essentially a game of mutual imitation, in which each individual tries to make its own state of motion as similar as possible to that of its neighbors [9]. From a physical point of view, such a mechanism is clearly suggestive of a ferromagnetic-like interaction: Focusing our attention on the direction of motion of each individual (the orientation of its velocity vector), such an imitation game amounts to a local interaction due to which each (normalized) velocity vector tends to align to those of its neighbors, much as classical Heisenberg spins tend to align to each other. At variance with standard ferromagnets, though, in collective motion the positions of the particles change in time, as they are carried around by their own velocities, thus creating a nonequilibrium feedback between the alignment degrees of freedom and the interaction network [20,33]. The simplest yet most illuminating model describing this core mechanism of 
collective motion was introduced by Vicsek and coworkers [34]; it describes a set of self-propelled particles that interact with each other in a ferromagnetic way,

$$
\begin{gathered}
\hat{\eta} \frac{d \boldsymbol{v}_{i}}{d t}=\hat{J} \sum_{j} n_{i j}(t) \boldsymbol{v}_{j}+\zeta_{i} \\
\frac{d \boldsymbol{r}_{i}}{d t}=\boldsymbol{v}_{i},
\end{gathered}
$$

where $\boldsymbol{r}_{i}$ is the position of particle $i, \boldsymbol{v}_{i}$ its velocity, and $n_{i j}(t)$ is the (short-ranged) adjacency matrix (who is neighbor of whom) at time $t$. The interaction between individuals is given by the ferromagnetic term in (4), where $\hat{J}$ gives the strength of the tendency to align to each other. ${ }^{2}$ Such alignment interaction is often called social force in the collective behavior literature [9]. In the Vicsek model, the speed is kept fixed, $\left|\boldsymbol{v}_{i}\right|=1$, which is the purpose of the cross products at the right-hand side of (4). The term $\zeta_{i}$ is a Gaussian white noise with variance

$$
\left\langle\zeta_{i}(t) \cdot \zeta_{j}\left(t^{\prime}\right)\right\rangle=2 d \hat{\eta} T \delta_{i j} \delta\left(t-t^{\prime}\right),
$$

where $\hat{\eta}$ is a dissipation coefficient and $T$ is a generalized temperature measuring the strength of the noise.

The power of the Vicsek model is that it describes collective motion in its two different phases. When noise is low (or density is high, in the metric case [9]), the alignment interaction produces long-range order across the system, forming a polarized moving flock. The interesting thing is that such ordering also occurs in two dimensions, which would be forbidden by the Mermin-Wagner theorem [35] in an equilibrium ferromagnet with continuous symmetry; however, the Vicsek model has an off-equilibrium feedback between alignment and self-propulsion promoting long-range order [20]. On the other hand, when noise is large enough (or density is low, in the metric case [9]), the system is in a disordered (paramagnetic) phase, which reproduces quite well the statistical properties of real swarms. More precisely, it has been observed that natural midge swarms are disordered but highly correlated systems [36]; the static velocity correlations are (qualitatively at least) those given by the Vicsek model close to its ordering transition. Hence, the Vicsek model captures rather well the static correlation functions of collective motion for both flocks and swarms. Dynamics is more problematic, though, at both the qualitative and the quantitative level.

\section{B. The inertial spin model}

The first hint that the Vicsek equation of collective motion required some new ingredients came from experiments on flocks, in which it was observed that disturbances in the direction of motion of the birds (that is, turns) propagate linearly with very low dissipation [27]. Although the hydrodynamic field-theoretical description of the Vicsek model introduced by Toner and Tu [19] contains linearly propagating "sound" modes, caused by the feedback between local density and

\footnotetext{
${ }^{2}$ We use hatted parameters in the microscopic equations to distinguish them from their coarse-grained counterpart in the field equations later on.
}

phase fluctuations [37], experiments indicate that flocks follow a different mechanism: During the propagating event, the density displays very weak fluctuations, if any; moreover, the speed of the wave propagation has been found to increase with increasing polarization, a feature absent in the hydrodynamic theory of the Vicsek model [19] (see also the discussion in Ref. [26]). It was therefore suggested in Refs. [27] and [25] that Vicsek dynamics had to be complemented with some nondissipative inertial couplings between order parameter (the velocity) and a conjugate momentum, in order to reproduce the structure of a conservative Hamiltonian dynamics. The resulting microscopic dynamical equations give rise to the inertial spin model (ISM) of collective motion [25],

$$
\begin{aligned}
\frac{d \boldsymbol{v}_{i}}{d t} & =\frac{1}{\hat{\chi}} s_{i} \times v_{i}, \\
\frac{d \boldsymbol{s}_{i}}{d t} & =\boldsymbol{v}_{i} \times \hat{J} \sum_{j} n_{i j}(t) \boldsymbol{v}_{j}-\frac{\hat{\eta}}{\hat{\chi}} \boldsymbol{s}_{i}+\boldsymbol{v}_{i} \times \zeta_{i}, \\
\frac{d \boldsymbol{r}_{i}}{d t} & =\boldsymbol{v}_{i},
\end{aligned}
$$

where the new variable $s_{i}$ is the generator of the rotational symmetry of the interaction (and is therefore called spin, in an analogy with quantum mechanics) and is the cross product of the velocity and the generalized momentum canonically conjugate to the velocities $\boldsymbol{v}_{i}$. Associated to the momentum $s_{i}$ we have a generalized inertia, $\hat{\chi}$, which embodies the resistance of a particle to change its instantaneous radius of curvature [38]. One can show that, in the low noise, strongly polarized phase, the nondissipative coupling between spin and velocity of the ISM generates linear propagating modes of the velocity fluctuations, which match quite accurately the experimental results, including the key relation between speed of propagation and polarization [39].

In the absence of a dissipative term, the Hamiltonian structure of the ISM would conserve the total spin, as it happens for any generator of a symmetry. However, one can show that the spin is essentially the instantaneous curvature of the particle's trajectory [27], and hence a single particle (or bird, in a flock) would maintain its radius of curvature forever if the spin were strictly conserved. This is quite unrealistic. Rather, it seems reasonable to expect curvature (and therefore spin) to be dissipated in the long run in absence of interaction or external perturbations. For this reason, the ISM has also the dissipative term, $-\hat{\eta} \boldsymbol{s}_{i}$, and stochastic noise, $\zeta_{i}$, granting relaxation of the spin for large times. If dissipation is small, though, and the biological group has finite size, linear waves will still propagate across the system, before dissipation kicks in [25]. In other words, although in the hydrodynamic limit (infinitely large times and distances) the conservative Hamiltonian structure always becomes irrelevant, on the finite-time and finite-size scales typical of biological phenomena the interplay between velocity and spin has crucial consequences on signal propagation. Note, finally, that once dissipation is included in the equations, one can recover the Vicsek model as the overdamped limit of the ISM [25], which is quite reassuring.

The second hint that a model with nondissipative dynamics was required came from swarms. Swarms of insects are 
systems apparently completely different from flocks: They show no group-scale coordination, so that their net motion is zero: Swarms "dance" above some landmark in seemingly random fashion [13]. In fact, experiments on natural swarms [13] showed that these systems have strong velocity correlations, indicating that, despite the lack of long-range order, the individuals within these groups are interacting with each other rather intensely, hence driving the system close to an ordering transition; indeed, such static correlations were qualitatively similar to those developed by the Vicsek model at its critical point [36]. More recent experiments [22] showed that swarms in their natural environment exhibit another important property of classical statistical physics, namely dynamic scaling [3]: According to this law, the dynamic correlation function of a system close to the critical point obeys the following relations:

$$
\begin{gathered}
C(k, t)=C_{0}(k) F\left(t / \tau_{k}, k \xi\right), \\
\tau_{k}=k^{-z} f(k \xi),
\end{gathered}
$$

where $t$ is time, $k$ is momentum, $C_{0}$ is the static correlation function, $\tau_{k}$ is the relaxation time of mode $k, F$ and $f$ are well-behaved scaling functions, and $z$ is the dynamic critical exponent, ruling how space and time scale with each other. The key idea of dynamic scaling is that the only relevant scale in ruling both spatial and temporal behavior of a system close to the critical point is the correlation length $\xi$. For $k=0$, we obtain $\tau \sim \xi^{z}$, a property known as critical slowdown: A system strongly correlated in space must also be strongly correlated in time [23]. Experiments showed that swarms satisfy relations (8) with a dynamic critical exponent $1 \leqslant z \leqslant$ 1.3 (with the best collapse for $z=1.2$ ), whereas numerical simulations of the Vicsek model in $d=3$ give $z \approx 2$ [22]. It must be noted that $z=2$ is the exact value of the dynamical critical exponent for a purely dissipative free theory (Gaussian model) and that even in the interacting case the exponent receives only very small (two loops) corrections to the value 2 , if the dynamics has only dissipative terms (or even values larger than 2, as in the case of model B [23]). On the other hand, dynamical models with nondissipative inertial terms tend to have values of the exponent $z$ significantly smaller than 2, as a result of the interplay between order parameter and conjugate momentum [23]. Hence, the low value of $z$ in natural swarms was a further indication of the need for nondissipative dynamics also in these nonpolarized systems. Finally, dynamic relaxation in the Vicsek model has a classic exponential form [22], while natural swarms display a completely different shape, showing clear evidence of nondissipative inertial behavior for short times. More precisely, if we define the relaxation form factor [22], $h=\dot{C}(t / \tau) / C(t / \tau)$, in the limit $t / \tau \rightarrow 0$ we have that $h \rightarrow 1$ for the Vicsek model (as for any exponential correlation function), while experiments showed $h \rightarrow 0$ for natural swarms, as it would happen in a weakly damped harmonic system, where inertia dominates over dissipation [22]. Hence, the whole dynamical behavior of swarms seems to require the existence of nondissipative inertial terms in the equations of motion, which is exactly the extra ingredient the ISM has compared to the Vicsek model.
Our plan is therefore to study the critical dynamics of the ISM in its disordered yet near-critical phase to describe the collective behavior of natural swarms of insects, in order to try and reproduce the experimental results of Ref. [22]. Because the ISM was originally introduced to describe the dynamics of flocks, it has been studied extensively in its deeply ordered (i.e., polarized) phase, both numerically [25] and theoretically [39], while no previous studies of the ISM in the near-critical regime have been performed.

\section{Fixed network approximation}

Before we proceed with the coarse graining of the model, though, we need to decide whether to attack directly the full-fledged off-equilibrium problem, which includes the selfpropelled nature of collective motion, or whether we first take on the simpler (and yet nontrivial) equilibrium problem, in which particles sit on a fixed network and thus have a time-independent interaction matrix. For a number of reasons, we will follow the second strategy. The model we want to study differs from previous known cases in two main respects: (i) it contains nondissipative terms and effective friction, the interplay of which has never been studied before, not even at equilibrium, and (ii) the model is a self-propelled one, hence intrinsically off equilibrium, which may seem particularly important in the swarm phase, in which each particle changes the local neighbors quite rapidly. Our central experimental concern is to reproduce the correct dynamical critical exponent $z$ and the correct relaxation form factor of natural swarms. The fact that the self-propelled, off-equilibrium Vicsek model in its swarm phase gives exactly the same exponent and form factor as equilibrium fully dissipative models (as the classical Heisenberg model) suggests that self-propulsion is not the primary source of the anomalous critical dynamics of swarms. Moreover, we believe that having under control the equilibrium problem puts us in a more solid position to tackle the off-equilibrium one in the future, much as knowing the physics of the equilibrium $X Y$ and Heisenberg models has been fundamental to fully understanding and appreciating the Vicsek model. Hence, we will study a fixed-network version of the ISM, in which the particles belong to a lattice and the connectivity matrix does not depend on time. In this context, the order parameter no longer has the role of a velocity, so we will call it $\psi$, the generic symbol for the order parameter, and write the microscopic model as

$$
\begin{gathered}
\frac{d \boldsymbol{\psi}_{i}}{d t}=\frac{1}{\hat{\chi}} \boldsymbol{s}_{i} \times \boldsymbol{\psi}_{i} \\
\frac{d \boldsymbol{s}_{i}}{d t}=\boldsymbol{\psi}_{i} \times \hat{J} \sum_{j} n_{i j} \boldsymbol{\psi}_{j}-\frac{\hat{\eta}}{\hat{\chi}} \boldsymbol{s}_{i}+\boldsymbol{\psi}_{i} \times \zeta_{i} .
\end{gathered}
$$

The modulus of the order parameter is still fixed to $\left|\boldsymbol{\psi}_{i}\right|^{2}=1$ and the adjacency matrix $n_{i j}$ now corresponds to a fixed interaction network. Thanks to this approximation, the dynamical equations can now be rewritten in Hamiltonian terms,

$$
\begin{gathered}
\frac{d \boldsymbol{\psi}_{i}}{d t}=-\boldsymbol{\psi}_{i} \times \frac{\partial H}{\partial \boldsymbol{s}_{i}}, \\
\frac{d \boldsymbol{s}_{i}}{d t}=-\boldsymbol{\psi}_{i} \times \frac{\partial H}{\partial \boldsymbol{\psi}_{i}}-\hat{\eta} \frac{\partial H}{\partial \boldsymbol{s}_{i}}+\boldsymbol{\psi}_{i} \times \zeta_{i},
\end{gathered}
$$


with microscopic Hamiltonian,

$$
H=-\hat{J} \sum_{i, j} n_{i j} \boldsymbol{\psi}_{i} \cdot \boldsymbol{\psi}_{j}+\sum_{i} \frac{s_{i}^{2}}{2 \hat{\chi}} .
$$

This is the model we now proceed to coarse grain in order to obtain a dynamical field theory.

\section{COARSE-GRAINED FIELD THEORY}

\section{A. Equations of motion}

Since we are interested in describing the large-scale behavior of the system, it is convenient to pass from a microscopic description in terms of site-dependent variables to a field description, where we consider smoothly varying order parameter and spin fields, $\boldsymbol{\psi}(\boldsymbol{x}, t)$ and $\boldsymbol{s}(\boldsymbol{x}, t)$, obtained by coarse graining the original variables over a small spatial volume. Upon coarse graining, the original Hamiltonian (14) gives rise to an effective field Hamiltonian $\mathcal{H}[\boldsymbol{\psi}, \boldsymbol{s}]$ that - as in standard ferromagnetic systems — reads [40,41]

$$
\mathcal{H}[\boldsymbol{\psi}, s]=\int d^{d} x\left\{\frac{1}{2}(\nabla \boldsymbol{\psi})^{2}+\frac{1}{2} r_{0} \psi^{2}+u_{0} \psi^{4}+\frac{s^{2}}{2 \chi_{0}}\right\},
$$

where $r_{0}$ is the bare mass (negative in the ordered phase), $u_{0}$ is the bare static coupling constant, and $\chi_{0}$ is the effective inertia. Here, we note the gradient term comes from the mutual alignment interaction, which favors smoother configurations; the quadratic and quartic contributions for $\psi$ represent a confining potential and derive from the original constraint on the $\psi_{i}$ and the coarse-graining entropy, while the field $\boldsymbol{s}$ remains Gaussian as its microscopic counterpart.

When writing down the dynamical equation of motion for the fields, we need to take into account the presence of both the reversible and dissipative contributions present in the microscopic dynamics (12) and (13) and add the dissipative terms which may arise upon coarse graining. Under very general assumptions [23,42], we can therefore write

$$
\begin{gathered}
\frac{\partial \boldsymbol{\psi}}{\partial t}=-\Gamma_{0} \frac{\delta \mathcal{H}}{\delta \boldsymbol{\psi}}+g_{0} \boldsymbol{\psi} \times \frac{\delta \mathcal{H}}{\delta \boldsymbol{s}}+\boldsymbol{\theta}, \\
\frac{\partial \boldsymbol{s}}{\partial t}=\left(\lambda_{0} \nabla^{2}-\eta_{0}\right) \frac{\delta \mathcal{H}}{\delta \boldsymbol{s}}+g_{0} \boldsymbol{\psi} \times \frac{\delta \mathcal{H}}{\delta \boldsymbol{\psi}}+\zeta,
\end{gathered}
$$

where the Gaussian noises correlations are chosen to have a Boltzmann-like static probability distribution, i.e.,

$$
\begin{aligned}
& \left\langle\theta_{\alpha}(\mathbf{x}, t) \theta_{\beta}\left(\mathbf{x}^{\prime}, t^{\prime}\right)\right\rangle=2 \Gamma_{0} \delta_{\alpha \beta} \delta^{(d)}\left(\mathbf{x}-\mathbf{x}^{\prime}\right) \delta\left(t-t^{\prime}\right), \\
& \left\langle\zeta_{\alpha}(\mathbf{x}, t) \zeta_{\beta}\left(\mathbf{x}^{\prime}, t^{\prime}\right)\right\rangle=2\left(\eta_{0}-\lambda_{0} \nabla^{2}\right) \delta_{\alpha \beta} \delta^{(d)}\left(\mathbf{x}-\mathbf{x}^{\prime}\right) \delta\left(t-t^{\prime}\right) .
\end{aligned}
$$

Here, $\Gamma_{0}, \eta_{0}$, and $\lambda_{0}$ are the bare kinetic coefficient of the field $\psi$ and the bare friction coefficient and transport coefficient of the field $\boldsymbol{s}$, respectively, while $g_{0}$ is a mode-coupling constant that regulates the reversible dynamical terms and describes the symmetry properties relating the two fields: The fact that $s$ is the infinitesimal generator of rotations of $\psi$ is indeed specified by the Poisson commutation rules,

$$
\left\{\psi_{\alpha}, s_{\beta}\right\}=g_{0} \epsilon_{\alpha \beta \gamma} \psi_{\gamma}, \quad\left\{s_{\alpha}, s_{\beta}\right\}=g_{0} \epsilon_{\alpha \beta \gamma} s_{\gamma},
$$

where $\epsilon_{\alpha \beta \gamma}$ is the Levi-Civita antisymmetric symbol. ${ }^{3}$

The static properties of the model only depend on the Hamiltonian $\mathcal{H}$. For the field $\boldsymbol{\psi}$, they are therefore the same as in the Heisenberg model [23], with an ordering phase transition occurring for $r_{0}=r_{c}$. On the other hand, at the static level $s$ is a trivial, purely massive Gaussian field. Since there is no static coupling between this field and the order parameter, the inertia $\chi_{0}$ will not acquire any perturbative contributions; hence, in order to simplify our notation, we choose the units of $s$ such that $\chi_{0}=1$. The dynamic properties are ruled by the transport coefficient $\lambda_{0}$, the effective friction $\eta_{0}$, the kinetic coefficient $\Gamma_{0}$, and the dynamic coupling constant $g_{0}$; these quantities will take perturbative contributions arising from the dynamic interaction between $s$ and $\boldsymbol{\psi}$, which is ruled by $g_{0}$.

Equations (15) and (16) have two additional dissipative terms compared to the microscopic theory of Eqs. (12) and (13), namely $-\Gamma_{0} \delta \mathcal{H} / \delta \boldsymbol{\psi}$ and $\lambda_{0} \nabla^{2} \delta \mathcal{H} / \delta \boldsymbol{s}$. The first term actually contains two contributions: first, a derivative of the confining potential, $\psi^{2}+\psi^{4}$, which is the coarse-grained analog of the microscopic sharp constraint, $\left|\boldsymbol{\psi}_{i}\right|^{2}=1$; and second, a diffusive piece, $\Gamma_{0} \nabla^{2} \boldsymbol{\psi}(\mathbf{x}, t)$, which derives from a loss of reversibility due to the coarse graining and which describes the role of the fluctuations of the order parameter in the relaxation process. Even though such fluctuations are negligible in the low-temperature phase (where we recover the microscopic theory with $\Gamma_{0}=0$ ), they are crucial when considering the system close to the critical point. For this reason, even though we neglected the Laplacian of the order parameter in our previous analysis in the deeply ordered phase [39], we need to take it into account in the present study of the critical regime.

On the other hand, the origin of the spin transport term, $\lambda_{0} \nabla^{2} s(\mathbf{x}, t)$, is perhaps less intuitive. In the microscopic model, the spin is dissipated by the friction through the term $-\hat{\eta} \boldsymbol{s}_{i}(t)$, and hence one might have expected just a term $-\eta_{0} s(\mathbf{x}, t)$ in the coarse-grained theory. Why then are we introducing the term in $\lambda_{0} \nabla^{2} s(\mathbf{x}, t)$ ? We will show in the following sections that, in the context of perturbation theory and the renormalization group, such a term arises naturally from the nonlinear interaction between the two fields once a momentum shell integration is performed. It is then necessary to include the transport coefficient $\lambda_{0}$ directly from the starting field equations.

We notice that for $\eta_{0}=0$, Eqs. (15) and (16) coincide with those of model $\mathrm{G}$ (antiferromagnet), or, in the planar case, of model E (liquid helium), which have a fully conserved spin dynamics and whose critical dynamical properties have been studied long ago in a series of seminal papers $[43,44]$. The renormalization group analysis described in the following sections will show that in an appropriate regime (when $\eta_{0}$ is

\footnotetext{
${ }^{3}$ Note that we reabsorbed the minus sign in front of the cross products in (12) and (13) into the definition of the coarse-grained dynamical coupling constant, $g_{0}$, so to obtain in (15) and (16) the same field-theory notation as the classic reference papers, Refs. [43] and [44].
} 
small), the ISM displays the same critical behavior as these fully conservative models.

\section{B. Free theory in Fourier space}

The starting point to build the perturbative expansion of the equations of motion is the free (or noninteracting) theory, which is obtained by setting to zero the nonlinear coupling constants, namely $g_{0}=0$ and $u_{0}=0$. In Fourier variables, hence using momentum $k$ and frequency $\omega$, the free equations of motion become

$$
\begin{aligned}
& -i \omega \boldsymbol{\psi}(\boldsymbol{k}, \omega)=-\Gamma_{0}\left(k^{2}+r_{0}\right) \boldsymbol{\psi}(\boldsymbol{k}, \omega)+\boldsymbol{\theta}(\boldsymbol{k}, \omega), \\
& -i \omega \boldsymbol{s}(\boldsymbol{k}, \omega)=-\left(\eta_{0}+\lambda_{0} k^{2}\right) \boldsymbol{s}(\boldsymbol{k}, \omega)+\boldsymbol{\zeta}(\boldsymbol{k}, \omega) .
\end{aligned}
$$

The free theory is linear and it is therefore possible to solve it exactly by inverting Eqs. (19) and (20),

$$
\begin{gathered}
\boldsymbol{\psi}(\boldsymbol{k}, \omega)=G_{0, \psi}(\boldsymbol{k}, \omega) \boldsymbol{\theta}(\boldsymbol{k}, \omega), \\
\boldsymbol{s}(\boldsymbol{k}, \omega)=G_{0, s}(\boldsymbol{k}, \omega) \boldsymbol{\zeta}(\boldsymbol{k}, \omega),
\end{gathered}
$$

where the free propagators (or Green's functions) are the inverse of the dynamical operators in Fourier space,

$$
\begin{gathered}
G_{0, \psi}^{-1}(\boldsymbol{k}, \omega)=-i \omega+\Gamma_{0}\left(k^{2}+r_{0}\right), \\
G_{0, s}^{-1}(\boldsymbol{k}, \omega)=-i \omega+\left(\eta_{0}+\lambda_{0} k^{2}\right) .
\end{gathered}
$$

The propagators describe the response of the fields to noise and to external perturbations [45]. We can also define the free dynamic correlation functions,

$$
\begin{gathered}
C_{0, \psi}(k, \omega)=\langle\boldsymbol{\psi}(\boldsymbol{k}, \omega) \boldsymbol{\psi}(-\boldsymbol{k},-\omega)\rangle, \\
C_{0, s}(k, \omega)=\langle\boldsymbol{s}(\boldsymbol{k}, \omega) \boldsymbol{s}(-\boldsymbol{k},-\omega)\rangle .
\end{gathered}
$$

By using (21) and (22), and the noise correlators (17), we get the relations

$$
\begin{gathered}
C_{0, \psi}=2 \Gamma_{0}\left|G_{0, \psi}\right|^{2}, \\
C_{0, s}=2\left(\eta_{0}+\lambda_{0} k^{2}\right)\left|G_{0, s}\right|^{2} .
\end{gathered}
$$

These four quantities, propagators and correlation functions, are the building blocks of the perturbative expansion.

Calculations in the RG context are carried out in Fourier space, and hence all relevant integrals are performed over the momentum $k$. The lowest extreme of integration is $k=0$ (or of order $1 / L$ if the system is finite), whereas the upper extreme is a momentum scale-the so-called cutoff, $\Lambda$ corresponding to the inverse of the length scale over which the coarse graining has been performed. Practically speaking, if the continuous field has been obtained by averaging the microscopic variables on a volume of linear size $\mathcal{L}$, then $\Lambda=1 / \mathcal{L}$. The coarse graining is performed over a scale larger than the lattice spacing $a$; in practice, though, $\mathcal{L}$ is still a microscopic length scale of the system, so that, broadly speaking, one often assumes that $\Lambda$ is of order $1 / a$.

The cutoff is an arbitrary scale, which therefore appears as an unknown parameter of the theory. In fact, all bare parameters, $r_{0}, u_{0}, \Gamma_{0}, \lambda_{0}, \eta_{0}, g_{0}$, depend on $\Lambda$ and therefore are all equally unknown. As we shall see, the central idea of the renormalization group is to exploit constructively the arbitrariness of $\Lambda$, by studying how the bare parameters change when $\Lambda$ is changed; from this flow, the critical properties of the theory will emerge.

\section{RENORMALIZATION GROUP IN MOMENTUM SHELL}

Broadly speaking, the renormalization group is a set of symmetry transformations that are useful to determine the scale invariance properties of a system at its critical point [5]. An RG transformation unfolds through two stages: (i) integration of the short wavelength details and (ii) rescaling of length and time. The first operation amounts to integrating the fields over large values of the momentum, $\Lambda / b<k<\Lambda$, where $b$ is a rescaling factor larger than but close to, 1 . This integration interval is the so-called momentum shell. The effect of integration is to shift the cutoff from $\Lambda$ to $\Lambda / b$; the $\mathrm{RG}$ idea is that the long-distance physics of a system close to the critical point, where the correlation length is large, cannot change due to an arbitrary change of the cutoff. Hence, the second stage consists in rescaling space (and consequently time) in such a way as to formally restore the original cutoff $\Lambda$ and to compare the newly obtained equations to the original ones. The compound effect of these two stages is to effectively change the parameters that appear in the equations of motion, hence determining a flow in the space of parameters. At the critical point, where the correlation length is infinite, the $R G$ transformation must have left the system exactly the same and therefore the fixed points of the RG flow provide all the important information on the large-scale physical properties of the system.

The RG technique is now standard and discussed in the literature both for static and dynamical problems [24,42,46,47]. In this section, we adopt a momentum shell renormalization scheme [5], as this is the approach that was used in the original papers on critical dynamics $[23,43,48]$. This will allow us to immediately spot differences with respect to the fully conservative case [43]. In Sec. VI, we will illustrate how the same results can be obtained using a Callan-Symanzik approach, more common in recent applications of the dynamical renormalization group [42].

\section{A. Integration of the short-wavelength details}

In the first stage of the RG, we integrate out short wavelength fluctuations, namely modes with $\Lambda / b<k<\Lambda$. This operation (described in Appendix D) leads to a new effective theory that only depends on fields fluctuating over larger wavelengths, $k<\Lambda / b$. In the free theory, modes at different wave vectors are independent, so this operation has no practical effects. On the other hand, when nonlinear interactions are present, the coupling between long and short wavelength modes makes it impossible to carry out exactly this operation, which therefore requires a perturbative expansion that we describe in detail in Appendix A. The bottom line result of shell integration is to produce additional terms in the equations of motion that effectively modify the coefficients of both the linear and the nonlinear terms. We start with the linear dynamical coefficients, namely $\Gamma_{0}, \lambda_{0}$, and $\eta_{0}$. These parameters are contained in the free propagators, Eqs. (23) and 
(24). Because of the shell integration, the propagators acquire some new contributions, the so-called on-shell self-energies, $\Sigma_{b}$ and $\Pi_{b}$, so we can write

$$
\begin{aligned}
& G_{\psi}^{-1}(\mathbf{k}, \omega)=-i \omega+\Gamma_{0}\left(k^{2}+r_{0}\right)-\Sigma_{b}(\mathbf{k}, \omega), \\
& G_{s}^{-1}(\mathbf{k}, \omega)=-i \omega+\left(\eta_{0}+\lambda_{0} k^{2}\right)-\Pi_{b}(\mathbf{k}, \omega) .
\end{aligned}
$$

As we said, to compute the self-energies one uses perturbation theory. To carry out the expansion, we follow the generating functional approach of Martin-Siggia-Rose [24,44,49], where averages of physical observables over the stochastic dynamics are rewritten as thermal averages over a functional measure. The complication is that new auxiliary fields must be introduced and the effective field theory therefore involves four fields rather than two. The advantage is that the standard Feynman technique can be used to perform a diagrammatic expansion, and perturbation theory can be carried out in the same way as in equilibrium statistical field theory. The details can be found in Appendix A. To one loop order, the selfenergies read

$$
\begin{gathered}
\Sigma_{b}(\mathbf{k}, \omega)=-2 g_{0}^{2} \int_{\Lambda / b}^{\Lambda} \frac{d^{d} p}{(2 \pi)^{d}} \frac{\left(k^{2}+r_{0}\right)}{\left(p^{2}+r_{0}\right)\left(-i \omega+\Gamma_{0}\left(p^{2}+r_{0}\right)+\lambda_{0}(\mathbf{k}-\mathbf{p})^{2}+\eta_{0}\right)}, \\
\Pi_{b}(\mathbf{k}, \omega)=-g_{0}^{2} \int_{\Lambda / b}^{\Lambda} \frac{d^{d} p}{(2 \pi)^{d}} \frac{\left[p^{2}-(\mathbf{k}-\mathbf{p})^{2}\right]^{2}}{\left(p^{2}+r_{0}\right)\left[(\mathbf{k}-\mathbf{p})^{2}+r_{0}\right]\left[-i \omega+\Gamma_{0}\left(p^{2}+(\mathbf{k}-\mathbf{p})^{2}+2 r_{0}\right)\right]} .
\end{gathered}
$$

The self-energies modify the poles of the propagators in the frequency plane, therefore affecting the way response and correlation functions decay in time. In particular, the $k \rightarrow 0$ and $\omega \rightarrow 0$ expansion of the self-energies and of their derivatives modify the kinetic and transport coefficients, so that we can define their renormalized values,

$$
\begin{gathered}
\left.\Gamma_{R} \equiv \frac{\partial G_{\psi}^{-1}}{\partial k^{2}}\right|_{\substack{k=0 \\
\omega=0}}=\Gamma_{0}\left(1-\left.\frac{1}{\Gamma_{0}} \frac{\partial \Sigma_{b}}{\partial k^{2}}\right|_{\substack{k=0 \\
\omega=0}}\right), \\
\left.\lambda_{R} \equiv \frac{\partial G_{s}^{-1}}{\partial k^{2}}\right|_{\substack{k=0 \\
\omega=0}}=\lambda_{0}\left(1-\left.\frac{1}{\lambda_{0}} \frac{\partial \Pi_{b}}{\partial k^{2}}\right|_{\substack{k=0 \\
\omega=0}}\right), \\
\left.\eta_{R} \equiv G_{s}^{-1}\right|_{\substack{k=0 \\
\omega=0}}=\eta_{0}\left(1-\left.\frac{1}{\eta_{0}} \Pi_{b}\right|_{\substack{k=0 \\
\omega=0}}\right) .
\end{gathered}
$$

First of all, we notice an important point: From (32), we immediately see that $\Pi_{b}(k=0)=0$, and therefore we conclude that the effective friction $\eta_{0}$ has no perturbative corrections. As discussed in Appendix A, from the diagrammatic point of view this is a consequence of the structure of the vertex, which makes all perturbative contributions to $\Pi_{b}(k=0)$ equal to zero; therefore, this result is valid to all orders in perturbation theory. Physically, this fact is a consequence of the rotational symmetry of the theory: Even though $\eta_{0}$ breaks the conservation law of the spin, the symmetry is still at work, implying that it is impossible for the conservative theory to produce a nonconservative friction through coarse graining.

The accuracy of the perturbation expansion is increased by substituting the bare mass $r_{0}$ with its renormalized value $r$ [47], which represents the inverse static susceptibility and goes to zero when the systems approaches the critical temperature. Since we are interested in the critical behavior, from now on we will evaluate all integrals at $r=0$, namely at the critical point; given that integrals are on the shell there are no infrared singularities and the self-energies are finite. We thus have

$$
\Gamma_{R}=\Gamma_{0}\left[1+2 \frac{g_{0}^{2}}{\Gamma_{0}} \int_{\Lambda / b}^{\Lambda} \frac{d^{d} p}{(2 \pi)^{d}} \frac{1}{p^{2}\left[\left(\Gamma_{0}+\lambda_{0}\right) p^{2}+\eta_{0}\right]}\right],
$$

$$
\begin{gathered}
\lambda_{R}=\lambda_{0}\left[1+\frac{1}{2} \frac{g_{0}^{2}}{\Gamma_{0} \lambda_{0}} \frac{4}{d} \int_{\Lambda / b}^{\Lambda} \frac{d^{d} p}{(2 \pi)^{d}} \frac{1}{p^{4}}\right], \\
\eta_{R}=\eta_{0} .
\end{gathered}
$$

These equations show that there is a great difference in the role of the two parameters $\lambda_{0}$ and $\eta_{0}$. If we start from a frictionless model that has $\eta_{0}=0$, the coarse graining of the $\mathrm{RG}$ will not generate a friction through shell integration. On the contrary, even if we start from a model without spin transport coefficient, $\lambda_{0}=0$, integration over short wavelengths inevitably generates a transport term $\lambda_{R} \neq 0$. In other words, the interaction between the spin $s$ and the primary field $\psi$ generates a nonzero transport coefficient $\lambda_{R}$ even if $\lambda_{0}=0$ in the original microscopic theory. For this reason, we included from the outset the parameter $\lambda_{0}$ in the coarse-grained field equations. $4^{4}$

To make further progress, we must address a rather crucial algebraic detail. While the integral in (37) is straightforward, the one in (36) requires some care. The cutoff $\Lambda$ is large, while the rescaling factor $b$ is close to 1 ; hence, the shell integration is performed over large values of the internal momentum $p$. If the effective friction $\eta_{0}$ is finite (or zero), then the term of order $p^{2}$ dominates over $\eta_{0}$ at the denominator, so that the overall integrand will have a $1 / p^{4}$ behavior for large momentum. As we shall see later on, the hypothesis that $\eta_{0}<\infty$ is by no means harmless, and we shall need to return over this point. Yet, for now we will work under this hypothesis, and recognize that it is therefore convenient to rewrite the integral as

$$
\Gamma_{R}=\Gamma_{0}\left[1+2 \frac{g_{0}^{2}}{\Gamma_{0}\left(\Gamma_{0}+\lambda_{0}\right)} \int_{\Lambda / b}^{\Lambda} \frac{d^{d} p}{(2 \pi)^{d}} \frac{1}{p^{2}\left(p^{2}+\frac{\eta_{0}}{\Gamma_{0}+\lambda_{0}}\right)}\right]
$$

\footnotetext{
${ }^{4}$ In other words, $\eta_{0}=0$ is an RG fixed point (although unstable, as we shall see), whereas $\lambda_{0}=0$ is not.
} 
We can now change variables, defining $p=\Lambda x$, and obtain

$$
\begin{gathered}
\Gamma_{R}=\Gamma_{0}\left[1+2 \frac{g_{0}^{2} \Lambda^{d-4}}{\Gamma_{0} \lambda_{0}\left(1+\frac{\Gamma_{0}}{\lambda_{0}}\right)} \int_{1 / b}^{1} \frac{d^{d} x}{(2 \pi)^{d}} \frac{1}{x^{2}\left(x^{2}+\frac{\eta_{0}}{\lambda_{0}} \frac{\Lambda^{-2}}{1+\Gamma_{0} / \lambda_{0}}\right)}\right] \\
\lambda_{R}=\lambda_{0}\left[1+\frac{1}{2} \frac{g_{0}^{2} \Lambda^{d-4}}{\Gamma_{0} \lambda_{0}} \frac{4}{d} \int_{1 / b}^{1} \frac{d^{d} x}{(2 \pi)^{d}} \frac{1}{x^{4}}\right]
\end{gathered}
$$

It is now convenient to introduce a set of effective parameters, through which we can express these integrals in a simpler way (so to speak),

$$
f_{0}=\frac{g_{0}^{2}}{\lambda_{0} \Gamma_{0}} K_{d} \Lambda^{d-4}, \quad w_{0}=\frac{\Gamma_{0}}{\lambda_{0}}, \quad \mathcal{R}_{0}=\sqrt{\frac{\lambda_{0}}{\eta_{0}}},
$$

where $K_{d}$ is the unit sphere volume in dimension $d$. We can thus finally write the perturbative expression of all three kinetic parameters after shell integration,

$$
\begin{gathered}
\Gamma_{R}=\Gamma_{0}\left[1+\frac{2 f_{0}}{1+w_{0}} \int_{1 / b}^{1} \frac{d^{d} x}{x^{2}} \frac{1}{x^{2}+\left(\mathcal{R}_{0} \Lambda\right)^{-2}\left(1+w_{0}\right)^{-1}}\right] \\
=\Gamma_{0}\left[1+\frac{2 f_{0}}{1+w_{0}} X_{0} \log b\right], \\
\lambda_{R}=\lambda_{0}\left[1+\frac{f_{0}}{2} \int_{1 / b}^{1} \frac{d^{d} x}{x^{4}}\right]=\lambda_{0}\left[1+\frac{1}{2} f_{0} \log b\right], \\
\eta_{R}=\eta_{0},
\end{gathered}
$$

where we have introduced the dimensionless crossover parameter $X_{0}$,

$$
X_{0}=\frac{\left(\mathcal{R}_{0} \Lambda\right)^{2}\left(1+w_{0}\right)}{1+\left(\mathcal{R}_{0} \Lambda\right)^{2}\left(1+w_{0}\right)},
$$

and where to compute the integrals we have exploited the fact that in the limit $b \rightarrow 1$; that is, for an infinitesimal RG transformation, the shell becomes infinitesimal, so we have written the integrals as the shell thickness, $1-1 / b \sim \log b$, times the integrand evaluated at $x=1$.

The dimensionless parameter $w_{0}$ is rather harmless, and it will play only a moderate role in what follows; quite conveniently, it will remain finite in all fixed points we will find. On the other hand, $f_{0}$ is crucial, as it acquires the role of the effective dynamical coupling constant: The perturbative expansion, which naively one would think as a series in powers of $g_{0}$, is in fact a series in powers of $f_{0}$. From the dimensional form of $f_{0}$, and in particular from the fact that it contains a term $\Lambda^{d-4}$, RG connoisseurs can already deduce that the dynamical upper critical dimension of the theory will be $d_{c}=4$, the same as the static one. This will be made explicit once we will have solved the recursive RG equation for $f_{0}$ further on. We recall that this is a consequence of the fact that both integrands go like $1 / p^{4}$ for large momenta, hence giving a logarithmic behavior at $d=4$, and that, in turn, this follows from having assumed that $\eta_{0}$ is finite in the integral of Eq. (36). We will return to this hypothesis later on.

The second important effective parameter emerging from the equations is the length scale, $\mathcal{R}_{0}$, given by the ratio between the transport coefficient and the effective friction of the spin. Because of its definition, we can intuitively expect that if $\mathcal{R}_{0}$ is large $\left(\eta_{0}\right.$ small) the dynamics of the spin is ruled by a conservative diffusion mechanism. On the contrary, if $\mathcal{R}_{0}$ is small ( $\eta_{0}$ large), we expect the dynamics of the spin to be ruled by a dissipation mechanism. It is worth noticing that for $\mathcal{R}_{0}=\infty$, namely $\eta_{0}=0$, the crossover parameter is equal to 1 , and one correctly gets the same equations as the fully conservative model $\mathrm{G}$. On the other hand, when the conservation length scale is very small, $\mathcal{R}_{0} \sim 0$, which happens for $\eta_{0} \gg \lambda_{0}$, one gets $X_{0} \sim 0$, so that $\Gamma_{0}$ receives very weak perturbative corrections at one loop. We will return on this crucial point later on and we will see that this interplay between nonconservative dissipation and conservative transport coefficient of the spin plays a key role, giving rise to a nontrivial crossover between two different RG fixed points with different dynamic critical exponents.

Up to now, we focused on the coefficients of the linear terms in the equations of motion, $\Gamma_{0}, \lambda_{0}, \eta_{0}$. However, in general shell integration produces corrections to all terms, including the nonlinear ones. Therefore, the dynamical coupling constant, $g_{0}$, could in principle get a perturbative correction from shell integration, in particular from the renormalized vertex. However, it can be shown that-due to the structure of the interaction vertices - there are no perturbative corrections at all orders (see Appendix B about vertex corrections),

$$
g_{R}=g_{0} .
$$

As in the case of the lack of corrections to $\eta_{0}$, this result is a consequence of the symmetry properties of the system. Although the global spin is not conserved in our case, the field $s$ is the generator of the rotational symmetry of the $\psi$ field, and this symmetry generates Ward identities that protect $g_{0}$ at all orders (see Appendix C).

Finally, let us note that the static coupling constant, $u_{0}$, is renormalized as usual in the standard equilibrium theory [47]. However, the lowest order corrections to the dynamical coefficients due to the vertex $u_{0}$ are at two loops [23], whereas we perform here a one-loop calculation. Therefore, we do not need to address static renormalization any further in what follows. This is actually a nice feature of all theories with mode-coupling terms: The dynamical vertex is triple (see Appendix A), and hence one obtains very sizable corrections to the critical exponents already at one loop order, without using the two-loop corrections of the static vertex.

\section{B. Rescaling of space and time}

After integration over the shell, we are left with a theory which has new renormalized parameters, and also a new, smaller cutoff, $\Lambda / b$. In order to compare the new theory with the old one, and therefore to be able to write a set of recursive equations for the parameters, we rescale space, and therefore momentum $k$, by a factor $b$, in such a way to formally restore the original cutoff, $\Lambda / b \rightarrow \Lambda$. It must be noted that frequency does not have a similar cutoff, and hence in principle we would have no formal need to rescale $\omega$. However, this is deceiving: In order to reabsorb all powers of $b$ in the novel equations of motion, one can see that it is necessary also to rescale frequency [23]. Physically, this means that the rescaling of space and time cannot proceed independently: Space and time are tied together by the-yet 
unknown - dynamic critical exponent $z$, through the following rescaling relations,

$$
k \rightarrow b k, \quad \omega \rightarrow b^{z} \omega .
$$

Because of the nonlinear form of the equations of motion, we do not know a priori how the spatial integration affects the dynamics and we therefore allow for a generic dynamic $z$. As we shall see, its value determines how the order parameter relaxes close to criticality.

Rescaling momentum and frequency actually means changing physical units, and hence each parameter will also rescale according to its naive physical dimensions, which can be expressed in powers of $b$ in the following way:

$$
\begin{gathered}
\Gamma_{0} \rightarrow b^{z-2} \Gamma_{0}, \quad \lambda_{0} \rightarrow b^{z-2} \lambda_{0}, \\
\eta_{0} \rightarrow b^{z} \eta_{0}, \quad g_{0} \rightarrow b^{z-d / 2} g_{0} .
\end{gathered}
$$

Because the perturbative contribution of the shell integrals are given in terms of the effective parameters, $f_{0}, w_{0}, \mathcal{R}_{0}$, it is also necessary to write their corresponding rescaling laws:

$$
f_{0} \rightarrow b^{4-d} f_{0}, \quad w_{0} \rightarrow w_{0}, \quad \mathcal{R}_{0} \rightarrow b^{-1} \mathcal{R}_{0} .
$$

For $d>4$, the naive dimension of the effective coupling constant $f_{0}$ becomes negative; as we shall see, this implies that at its nontrivial fixed point the effective coupling constant will be of order $\epsilon$, with

$$
\epsilon=4-d,
$$

as it happens to the static coupling constant, $u_{0}$ [8], confirming the fact that the dynamical upper critical dimension is $d_{c}=4$.

We notice that, in general, shell integration and rescaling of $k$ and $\omega$ imply also a rescaling of the fields. However, once again we remark that the current calculation is at one-loop level, whereas the fields acquire a nontrivial scaling contribution only at two-loop level. For this reason, the anomalous dimension of the field $\psi$ (normally called $\eta$ and not to be confused with the friction) will be set to zero in the present calculation.

\section{Renormalization group recursive equations}

The two stages described above, shell integration and rescaling, must now be put together to define one step of the RG transformation; in this step, a generic parameter $\mathcal{P}$ is brought from its initial bare value, $\mathcal{P}_{0}$, to a new value, $\mathcal{P}_{1}$ through an RG equation with the structure,

$$
\mathcal{P}_{1}=b^{D_{\mathcal{P}}} \mathcal{P}_{0}\left(1+\delta_{\mathcal{P}} \log b\right)
$$

where the power of $b$ comes from the rescaling step, so that $D_{\mathcal{P}}$ is the physical dimension of $\mathcal{P}$, whereas the term in the bracket comes from the shell integration. For an infinitesimal RG transformation, $b \sim 1$; hence, we can write $1+\delta_{\mathcal{P}} \log b=$ $b^{\delta_{\mathcal{P}}}$, so that $\delta_{\mathcal{P}}$ is an effective correction to the naive scaling dimension $D_{\mathcal{P}}$ of the parameter. Of course, $\delta_{\mathcal{P}}$, which is the result of the shell integration, will depend on all the other parameters of the theory. One can then iterate this step $l$ times, giving rise to a recursive $R G$ equation for $\mathcal{P}$,

$$
\mathcal{P}_{l+1}=b^{D_{\mathcal{P}}} \mathcal{P}_{l}\left(1+\delta_{\mathcal{P}_{l}} \log b\right),
$$

where we emphasize that all integrals that appear in the right-hand side through the factor $\delta_{\mathcal{P}_{l}}$ must be evaluated at the running value of the parameters, namely at their value at the RG step $l$, whereas the naive physical dimension $D_{\mathcal{P}}$ is fixed once and for all. By using this procedure for the dynamical parameters of our theory, we obtain the following $\mathrm{RG}$ recursive relations:

$$
\begin{gathered}
\Gamma_{l+1}=b^{z-2} \Gamma_{l}\left(1+\frac{2 f_{l}}{1+w_{l}} X_{l} \log b\right), \\
\lambda_{l+1}=b^{z-2} \lambda_{l}\left(1+\frac{1}{2} f_{l} \log b\right), \\
\eta_{l+1}=b^{z} \eta_{l}, \\
g_{l+1}=b^{z-d / 2} g_{l},
\end{gathered}
$$

and we recall that we are working at $T=T_{c}$, namely on the critical manifold. From these equations, we can finally write a closed set of recursive relations for the effective coupling constant $f_{l}$, the dimensionless parameter $w_{l}$, and the conservation length scale $\mathcal{R}_{l}$,

$$
\begin{aligned}
f_{l+1} & =f_{l} b^{\epsilon}\left[1-f_{l}\left(\frac{1}{2}+\frac{2 X_{l}}{1+w_{l}}\right) \log b\right], \\
w_{l+1} & =w_{l}\left[1-f_{l}\left(\frac{1}{2}-\frac{2 X_{l}}{1+w_{l}}\right) \log b\right], \\
\mathcal{R}_{l+1} & =\mathcal{R}_{l} b^{-1}\left[1+\frac{1}{4} f_{l} \log b\right],
\end{aligned}
$$

where $X_{l}$ depends on $\mathcal{R}_{l}$ and $w_{l}$ through Eq. (46). We note that the full scaling dimension of the conservation length scale $\mathcal{R}$ is determined by its naive dimension, $b^{-1}$, plus a perturbative contribution, $1+\frac{1}{4} f_{l} \log b=b^{\frac{1}{4} f_{l}}$, hence developing an anomalous scaling dimension that will be crucial in ruling the crossover.

The derivatives of $f, w$, and $\mathcal{R}$ with respect to $(-\log b)$ are called $\beta$ functions and measure how the parameters change when performing an infinitesimal RG transformation, ${ }^{5}$

$$
\begin{aligned}
& \beta_{f}=-f\left[\epsilon-f\left(\frac{1}{2}+\frac{2 X}{1+w}\right)\right], \\
& \beta_{w}=w f\left[\frac{1}{2}-\frac{2 X}{1+w}\right], \\
& \beta_{\mathcal{R}}=\mathcal{R}\left[1-\frac{1}{4} f\right] .
\end{aligned}
$$

The zeros of these functions define the fixed points of the RG flow and thus have a crucial role in the theory. The $\beta$ functions also will provide a link between the momentum shell RG approach followed so far and the Callan-Symanzik approach described in Sec. VI.

\footnotetext{
${ }^{5}$ Usually, in the momentum shell scheme $\beta$ functions are defined as derivatives with regard to $\log b$; however, in that way one ends up with the opposite sign as compared to the usual definition in the Callan-Symanzik approach, where they are defined as derivatives with regard to the arbitrary momentum scale ( $\mu$, which is morally $1 / b)$. We use this convention so as to obtain in the end the same $\beta$ functions in both calculations.
} 


\section{Fixed points and dynamic critical exponent}

The fixed point values of the RG equations (that we are going to indicate with an asterisk) rule the critical behavior of the system. The exponent $z$ can be found by requiring that the fixed point value of the kinetic coefficient of $\psi$, namely $\Gamma^{*}$, is finite [23,24]. This condition, as we shall see, is what we need to investigate the relaxation behavior of the field $\psi$ close to criticality. From Eq. (55), we obtain $z$ as

$$
\Gamma^{*}=\mathcal{O}(1) \quad \Rightarrow \quad z=2-\frac{2 f^{*}}{1+w^{*}} X^{*} .
$$

The dynamic critical exponent is therefore given by the fixed point values of the parameters $f, w$, and $X$.

From the corresponding recursion equation, (59), it is evident that $\mathcal{R}$ can have two fixed points, namely

$$
\mathcal{R}^{*}=0, \quad \mathcal{R}^{*}=\infty .
$$

Since the fixed point of $f$ is expected to be of order $\epsilon$ [see Eqs. (60)], the scaling dimension of $\mathcal{R}$ is negative. Therefore, the $\mathcal{R}^{*}=0$ fixed point is IR stable while the $\mathcal{R}^{*}=\infty$ fixed point is IR unstable: Any large, but finite, initial value of $\mathcal{R}_{0}$, decreases under the RG equation (59), driving the system to the $\mathcal{R}^{*}=0$ fixed point. Inserting back the possible values of $\mathcal{R}^{*}$ in the other equations, we therefore find two fixed points for the global set of parameters.

\section{The IR-unstable conservative fixed point}

The first fixed point with $\mathcal{R}^{*}=\infty$ and $X^{*}=1$, which we call IR unstable (or conservative), is

$$
f^{*}=\epsilon, \quad w^{*}=3, \quad \mathcal{R}^{*}=\infty, \quad X^{*}=1, \quad \Longrightarrow z=d / 2 .
$$

This fixed point describes a dynamics with $z=d / 2$, typical of conservative models such as models $G$ and $E$ [43]. Indeed, $\mathcal{R}^{*}=\infty$ implies $\eta^{*}=0$ : The spin is not damped at all, so that dissipation becomes irrelevant and the conservation law expressed by the symmetries of the Hamiltonian rules the dynamics at all scales. If the system has $\mathcal{R}_{0}=\infty$ (i.e., $\eta_{0}=0$ ), the RG flow will converge to this fixed point, the only stable one for zero dissipation. However, as mentioned above, any other value of $\mathcal{R}_{0}$ will cause the flow to eventually converge to the other fixed point.

\section{The IR-stable dissipative fixed point}

The second fixed point is characterized by $\mathcal{R}^{*}=0$, or equivalently $X^{*}=0$, and we call it IR stable (or dissipative):

$$
f^{*}=2 \epsilon, \quad w^{*}=0, \quad \mathcal{R}^{*}=0, \quad X^{*}=0, \quad \Longrightarrow z=2 .
$$

In this case, dissipation takes over $\left(\eta^{*}=\infty\right)$ and the dynamic critical exponent that we obtain is $z=2$, which is common for models with a completely dissipative dynamics [23]. What we have depicted here is a scenario that includes the presence of two fixed points with different dynamical behaviors and different dynamic critical exponents, namely $z=d / 2$ (conservative dynamics) and $z=2$ (dissipative dynamics). Even though one of the fixed points is unstable along one direction, it is stable along the others and-as it will be discussed in the next section-it can rule the RG flow at intermediate iterations. In other terms, there is a crossover in the RG flow
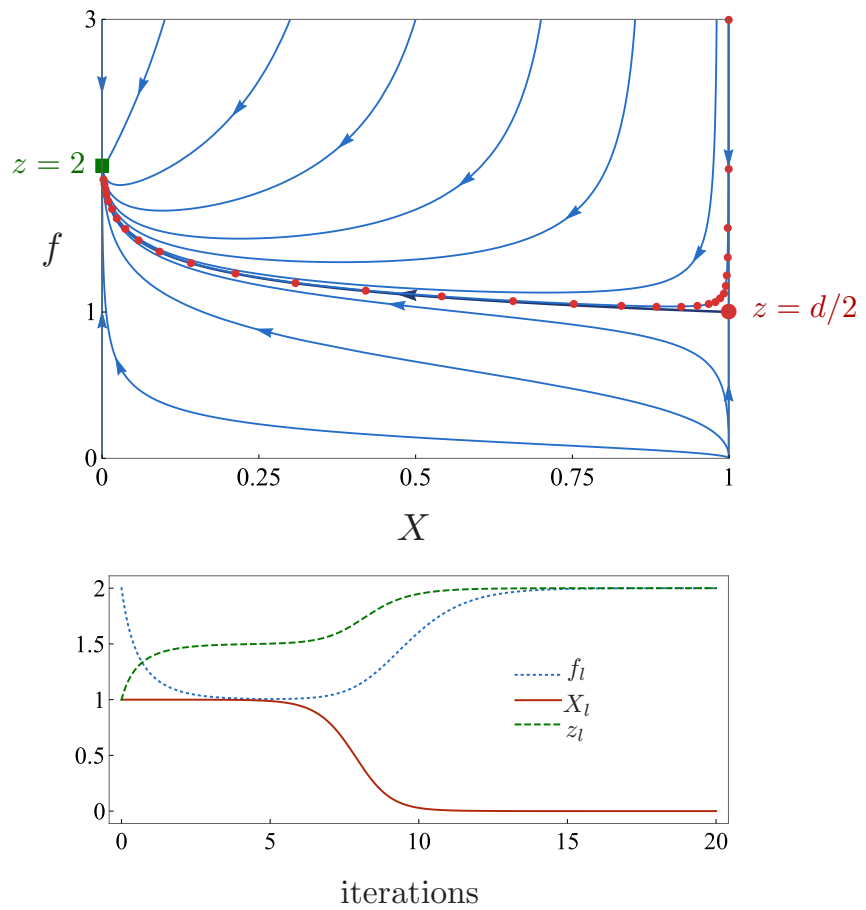

FIG. 1. Renormalization group flow and crossover. Top: Flow diagram on the $\left(X_{l}, f_{l}\right)$ plane for $d=3$. When the initial friction $\eta_{0}$ is small, $X_{0} \sim 1$, the flow converges toward the unstable fixed point, $z=d / 2$, and remains in its proximity for many iterations, before crossing over to the stable $z=2$ fixed point. Bottom: running parameters and critical exponent $z$ as a function of the iteration step along a flow line at small $\eta_{0}$. The initial values of the parameters are $f_{0}=2, X_{0}=0.9999$, and $w_{0}=3$.

in parameter space and, as a consequence, also in the behavior of physical observables.

\section{RENORMALIZATION GROUP CROSSOVER}

\section{A. RG flow on the critical manifold}

To investigate the dynamic crossover, we studied the RG flow from a numerical point of view. In the limit of infinitesimal RG transformations ( $b \rightarrow 1$ ), the recursion relations (59) become a system of coupled differential equations. We introduce the continuous variable $x=l \log b$; Eqs. (59) can then be rewritten in the continuum limit (replacing, for instance, $\left.f(x)=f_{l}\right)$ :

$$
\begin{gathered}
f^{\prime}(x)=\beta_{f}(f, w, \mathcal{R}), \\
w^{\prime}(x)=\beta_{w}(f, w, \mathcal{R}), \\
\mathcal{R}^{\prime}(x)=\beta_{\mathcal{R}}(f, w, \mathcal{R}),
\end{gathered}
$$

where the prime stands for a derivative with respect to $x$.

The set of Eqs. (63) can be studied numerically, for any given initial condition. In Fig. 1 (upper panel), we show the resulting flow in the $(X, f)$ plane, each line corresponding to a different set of initial values of $\eta_{0}, \lambda_{0}, \Gamma_{0}$ (and therefore of $f_{0}$ and $X_{0}$ ). The flow always proceeds from the conservative to the dissipative fixed point, as expected. However, how fast it does so depends on the initial condition $X_{0}$. When this value is close to 1 , which means that the friction $\eta_{0}$ is small, the 
flow of parameters approaches the $z=d / 2$ fixed point and remains close to it for many RG iterations. Then, it eventually moves toward the stable fixed point with $z=2$. In the lower panel of Fig. 1, we show the same dynamic crossover in terms of $z$ of the coupling constant $f$ and of $X$. From this figure, we clearly see that there is a well-defined intermediate regime where the flow is regulated by the unstable fixed point, the parameter $X$ driving the dynamic critical exponent from one value $(z=d / 2)$ to the other $(z=2)$.

\section{B. Crossover in the critical dynamics}

We have discussed so far the crossover along the RG flow in parameter space. This crossover has important observable consequences in the relaxational behavior of the system. In the previous section, we showed that there is a parameter with the dimensions of a length scale, $\mathcal{R}$, which plays a crucial role in the RG flow. As we shall see, it is precisely the interplay between $\mathcal{R}$ and the relevant physical length scales of the system that determines the way it relaxes.

\section{Crossover in $k$ at $\xi=\infty$}

Let us start by considering the system at the critical point $(r=0)$. In this case, the correlation length is infinite and the only physically relevant length scale is that at which we are observing the system, namely $1 / k$. To study relaxation at this scale, we can look at the correlation function of the order parameter, which is directly connected to the dynamic critical exponent through the dynamic scaling hypothesis (8). Since we have been working in frequency space, it is convenient to rewrite Eq. (8) in terms of frequencies [16]

$$
\begin{aligned}
C(k, \omega) & =\frac{2 \pi}{\omega_{c}(k)} C_{0}(k) H\left(\omega / \omega_{c}(k), k \xi\right), \\
\omega_{c}(k) & =k^{z} h(k \xi),
\end{aligned}
$$

where $H$ and $h$ are well-behaved scaling functions (the first normalized to 1 to ensure the static $\left.\operatorname{limit}^{6}\right)$ and $\omega_{c}(k)$ is the characteristic frequency of mode $k$ [the inverse of the characteristic time $\tau_{k}$ of Eq. (8)]. For $\xi=\infty$ and for small $k$, Eq. (64) gives

$$
\omega_{c}(k) \sim k^{z} .
$$

Since there are two possible values of $z$, one can wonder at this point which one to consider in this relationship. It turns out that this depends on $k$ versus $\mathcal{R}$. To see this, we note that the characteristic frequency is the pole of the propagator of the field $\psi$, i.e., the frequency satisfying $G_{\psi}^{-1}\left(k, \omega_{c}\right)=0$. We can therefore study its infrared behavior by looking at $G_{\psi}^{-1}$ at small $k$. This is very convenient because-by constructionpropagators along the RG flow are related to each other, and we can link what happens in parameter space to the physical behavior of the system.

\footnotetext{
${ }^{6}$ The prefactor $2 \pi / \omega_{c}(k)$ in the right-hand side of Eq. (64) ensures that, when integrating over frequencies, the condition $C(k, t=0)=$ $C_{0}(k)$ is satisfied.
}

At every step $l$ of the RG, the physical propagator verifies the relation

$$
G_{\psi}(k, \omega, \mathcal{P})=\left(b^{l}\right)^{z_{l}} G_{\psi}\left(b^{l} k, b^{l^{z_{l}}} \omega, \mathcal{P}_{l}\right),
$$

where with $\mathcal{P}_{l}$ we indicate the set of the parameters after $l$ steps of RG (and $\mathcal{P}=\mathcal{P}_{0}$ in the left-hand side). The scaling factor on the right side of (66) is just the scaling dimension of the propagator. What we are doing is to consider an initial point in parameter space corresponding to our physical system (left-hand side), and then follow the RG flow in the critical manifold starting at that point. As $l$ increases, the propagator on the right-hand side is evaluated at farther points along the $\mathrm{RG}$ line. Since we know that there is a crossover along the RG flow, we are writing this expression with a dynamic critical exponent, which explicitly depends on the recursion step $l$. If we choose $l$ such that $b^{l}=\Lambda / k$, i.e., the maximum possible value, the propagator satisfies

$$
G_{\psi}(k, \omega, \mathcal{P})=\left(\frac{\Lambda}{k}\right)^{z^{*}} G_{\psi}\left(\Lambda,\left(\frac{\Lambda}{k}\right)^{z^{*}} \omega, \mathcal{P}^{*}\right) .
$$

Here, we have evaluated the function on the right side at the fixed point values of the parameters $\mathcal{P}$. This is justified if $l$ is large enough to approach the vicinity of a fixed point (i.e., small $k$ ). From Eq. (67), we get for the characteristic frequency

$$
\begin{aligned}
G_{\psi}^{-1}\left(k, \omega_{c}, \mathcal{P}\right)=0 & \Leftrightarrow G_{\psi}^{-1}\left(\Lambda, \frac{\omega_{c} \Lambda^{z^{*}}}{k^{z^{*}}}, \mathcal{P}^{*}\right)=0 \\
& \Rightarrow \omega_{c}(k) \sim k^{z^{*}}
\end{aligned}
$$

Which one of the two fixed points is reached-and therefore the value of $z^{*}$-depends on the starting point (i.e., the set $\mathcal{P}$ ) and on the number of iterations. More precisely, the condition that discriminates between the two possible fixed points is

$$
\mathcal{R}_{l} \simeq \Lambda^{-1}
$$

because it determines the value of the variable $X_{l}$ in expression (61). Let us consider the situation, which interests us more, where the starting point of the flow is close to the IR-unstable fixed point. The initial value of $\mathcal{R}$ is therefore large, corresponding to a system with low $\eta_{0}$. If $\mathcal{R}_{l} \gg \Lambda^{-1}$ holds for all the iterations, the flow will explore only the neighborhoods of the unstable fixed point and the values of $\mathcal{P}^{*}$ in (67) are the ones of the conservative dynamics. Therefore, in this case

$$
\mathcal{R}_{l} \gg \Lambda^{-1} \Rightarrow \omega_{c} \sim k^{d / 2} \text {. }
$$

However, it may happen that, even starting at the same point in parameter space, the number of iterations is so large that eventually the condition $\mathcal{R}_{l} \ll \Lambda^{-1}$ becomes satisfied, and the flow approaches the stable fixed point corresponding to $z=2$. In this case,

$$
\mathcal{R}_{l} \ll \Lambda^{-1} \Rightarrow \omega_{c} \sim k^{2} .
$$

Since the number of iterations is fixed by the value of the wave number $k\left(b^{l}=\Lambda / k\right)$, the condition $\mathcal{R}_{l} \simeq \Lambda^{-1}$ can be translated into a condition on $k$. The recursion relation for the conservation length scale gives $\mathcal{R}_{l}=\mathcal{R}_{0} b^{l\left(-1+f^{*} / 4\right)}$; since we are considering a flow starting close to the conservative 
fixed point, we can set $f^{*}=\epsilon$, which gives the anomalous scaling dimension of $\mathcal{R}$ at the conservative fixed point,

$$
\mathcal{R} \sim b^{-d / 4},
$$

from which we see that the length scale $\mathcal{R}$ has a scaling dimension equal to its naive dimension at the upper critical dimension, $d=4$, as expected. From the relation $\mathcal{R}_{l}=$ $\mathcal{R}_{0} b^{l(-d / 4)}$, we can finally identify a threshold value $k_{c}$ marking the limit between the two different scenarios described above, namely

$$
k_{c}=\Lambda\left(\Lambda \mathcal{R}_{0}\right)^{-4 / d} .
$$

To summarize, we therefore find that at criticality the relaxation behavior of the order parameter-as captured by the critical exponent $z$-depends on the relation between the scale at which we observe the system and the value of the length scale $\mathcal{R}_{0}$, i.e.,

$$
\begin{aligned}
& k \ll \Lambda\left(\Lambda \mathcal{R}_{0}\right)^{-4 / d} \Rightarrow z=2, \\
& k \gg \Lambda\left(\Lambda \mathcal{R}_{0}\right)^{-4 / d} \Rightarrow z=d / 2 .
\end{aligned}
$$

We therefore have found the third nontrivial critical exponent of the theory, namely the crossover exponent [24],

$$
\kappa=4 / d,
$$

which, as we have seen, is intimately related to the anomalous dimension of the conservation length scale. In Fig. 2, we show the regions corresponding to the two dynamical behaviors in the $\left(k^{-1}, \mathcal{R}_{0}\right)$ plane.

\section{Crossover in $\xi$ at $k=0$}

In many cases, and in particular when looking at experimental data, real systems are not exactly at the critical point. For all practical purposes, we need to extract information on the critical behavior of the system also when its correlation length $\xi$ is finite, even if large. Predictions can be obtained following a reasoning much similar to the one above, but taking explicitly into account the dependence of the propagator on temperature, i.e., on the correlation length. Besides, since there is a characteristic length scale, it is convenient to set $k=0$. Instead of Eq. (66), the relevant equation for the propagators then becomes

$$
\begin{aligned}
G_{\psi}(\omega, \xi) & =G_{\psi}(k=0, \omega, \xi, \mathcal{P}) \\
& =\left(b^{l}\right)^{z_{l}} G_{\psi}\left(k=0, b^{z_{l}} \omega, \xi_{l}, \mathcal{P}_{l}\right) .
\end{aligned}
$$

What we are doing is, again, to consider a point in parameter space corresponding to our physical system and to relate the physical propagator with propagators of models along an RG line starting at that point. The difference with the previous case is that now the RG flow takes place off the critical manifold, and therefore not only the parameters change upon iteration but also the correlation length, i.e.,

$$
\xi_{l+1}=\xi_{l} / b,
$$

with $\xi_{0}=\xi$ (i.e., the correlation length of the physical system). We can choose the number $l$ of iterations such that $b^{l}=\xi \Lambda$. If $\xi$ is large enough that the system comes close to

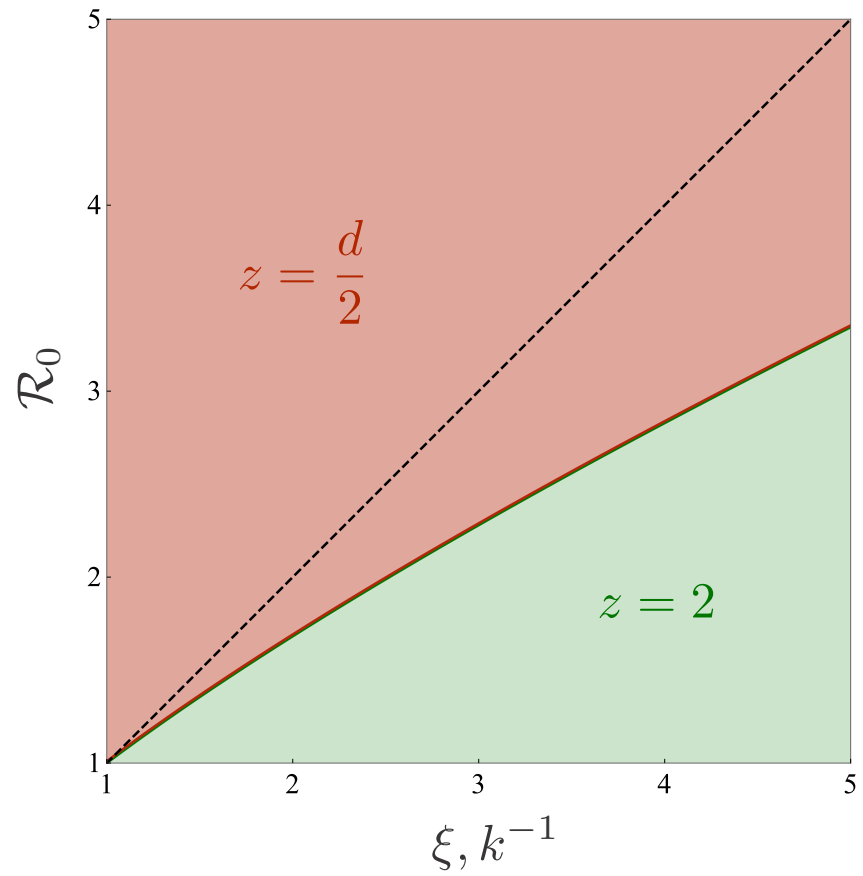

FIG. 2. Different critical regions. Different values of $k, \xi$, and $\mathcal{R}_{0}$ correspond to different critical behaviors. The dark red region corresponds to conservative critical dynamics with $z=d / 2$, while the light green region corresponds to dissipative critical dynamics with $z=2$. We set $\Lambda=1$ so that physical values for lengths are $k^{-1}>1$, $\xi>1$, and $\mathcal{R}_{0}>1$. On the critical manifold relaxation is studied in the $\left(k^{-1}, \mathcal{R}_{0}\right)$ plane: The two different regimes are separated by the curve $\mathcal{R}_{0}=k^{-d / 4}$. Off the critical manifold, relaxation is studied in the $\left(\xi, \mathcal{R}_{0}\right)$ plane: The two different regimes are separated by the curve $\mathcal{R}_{0}=\xi^{d / 4}$. The black dashed line represents, respectively, $\mathcal{R}_{0}=k^{-1}$ or $\mathcal{R}_{0}=\xi$. The figure refers to the $d=3$ case.

a fixed point, then the propagator satisfies the relation

$$
G_{\psi}(\omega, \xi)=(\Lambda \xi)^{z^{*}} G_{\psi}\left(k=0,(\Lambda \xi)^{z^{*}} \omega, \Lambda^{-1}, \mathcal{P}^{*}\right)
$$

Since the pole of the propagator for $k=0$ is the global characteristic frequency of the system, we immediately get the relaxation behavior as $\omega_{c}(\xi) \sim \xi^{-z^{\star}}$. As before, the value of $z$ depends on which one of the two fixed points is approached at the end of the RG flow after $l$ iterations. The discriminating condition is always $\mathcal{R}_{l} \simeq \Lambda^{-1}$; we therefore find

$$
\begin{aligned}
& \mathcal{R}_{l} \gg \Lambda^{-1} \Rightarrow \omega_{c} \sim \xi^{-d / 2}, \\
& \mathcal{R}_{l} \ll \Lambda^{-1} \Rightarrow \omega_{c} \sim \xi^{-2} .
\end{aligned}
$$

Since the number of iterations is fixed by $\xi$ (i.e., $b^{l}=\xi \Lambda$ ), the discriminating condition $\mathcal{R}_{l} \simeq \Lambda^{-1}$ now identifies a threshold value $\xi_{c}$ for the correlation length that can be obtained using the recursion relations of both $\mathcal{R}$ and $\xi$ :

$$
\xi_{c} \simeq\left(\mathcal{R}_{0} \Lambda\right)^{4 / d} \Lambda^{-1} .
$$

To conclude, we therefore find that critical slowdown is ruled by two different critical exponents depending on how large the correlation length is (i.e., how close the system is to the critical 
point) with respect to the conservation length scale $\mathcal{R}_{0}$, i.e.,

$$
\begin{array}{lll}
\xi \gg\left(\mathcal{R}_{0} \Lambda\right)^{4 / d} \Lambda^{-1} & \Rightarrow & z=2, \\
\xi \ll\left(\mathcal{R}_{0} \Lambda\right)^{4 / d} \Lambda^{-1} & \Rightarrow & z=d / 2,
\end{array}
$$

thus giving the same crossover exponent as in the $k$ description. A graphical representation of the different critical regimes can be found in Fig. 2. To summarize, it is therefore the interplay between the correlation length $\xi$ and the conservation length scale $\mathcal{R}_{0}$ that defines what kind of critical dynamical behavior is observed. We also note that-due to the nontrivial recursion relation for $\mathcal{R}$, see Eqs. (59)- these two length scales rescale differently upon RG transformations. As a consequence, the region corresponding to the conservative critical dynamics is larger than in the case of naive scaling.

\section{A new upper critical dimension}

So far we have been studying the RG flow in the vicinity of the conservative, $z=d / 2$, fixed point. Our original motivation was indeed to describe experimental findings on swarms of insects, where a low-dissipation critical dynamics has been observed. As we have seen, in the neighborhood of this fixed point, we have an upper critical dimension $d_{c}=4$ and the effective dynamic coupling constant is the parameter $f_{0}$. However, we also showed that the conservative fixed point is unstable, and hence the RG flow inevitably brings the system to the dissipative fixed point, $z=2$. The problem is that, in the vicinity of this fixed point, the on-shell self-energy $\Sigma_{b}$ has to be treated quite differently from the previous case, and $f_{0}$ does not play the role of the effective dynamic coupling constant anymore. Let us see this in more detail.

In proximity of the IR-stable fixed point, the running effective friction $\eta_{l}$ becomes very large, eventually diverging. In this regime, our previous assumption to have a mild, finite value of the friction $\eta_{l}$ in Eq. (36) must be revised, and the integral must be arranged differently:

$$
\begin{aligned}
\Gamma_{l+1} & =b^{z-2} \Gamma_{l}\left[1+2 \frac{g_{l}^{2}}{\Gamma_{l}} \int_{\Lambda / b}^{\Lambda} \frac{d^{d} p}{(2 \pi)^{d}} \frac{1}{p^{2}\left[\left(\Gamma_{l}+\lambda_{l}\right) p^{2}+\eta_{l}\right]}\right] \\
& =b^{z-2} \Gamma_{l}\left[1+2 \frac{g_{l}^{2}}{\Gamma_{l} \eta_{l}} \int_{\Lambda / b}^{\Lambda} \frac{d^{d} p}{(2 \pi)^{d}} \frac{1}{p^{2}\left(\frac{\Gamma_{l}+\lambda_{l}}{\eta_{l}} p^{2}+1\right)}\right] .
\end{aligned}
$$

We see that, as the running friction $\eta_{l}$ goes to infinity, approaching the stable fixed point, the large $p$ behavior of the integrand turns from $1 / p^{4}$ to $1 / p^{2}$, thus giving

$$
\Gamma_{l+1}=b^{z-2} \Gamma_{l}\left[1+2 \frac{g_{l}^{2}}{\Gamma_{l} \eta_{l}} \int_{\Lambda / b}^{\Lambda} \frac{d^{d} p}{(2 \pi)^{d}} \frac{1}{p^{2}}\right] .
$$

From this last equation, it is clear that, in the proximity of the IR-stable fixed point, the actual effective coupling constant in the perturbative expansion of $\Gamma_{l}$ is no longer $f_{l}$ but $q_{l}=g_{l}^{2} \Lambda^{2-d} / \Gamma_{l} \eta_{l}$, whose naive scaling dimension is $d-2$, not $d-4$; accordingly, the integral now has a logarithmic UV divergence at $d=2$. We conclude that the upper critical dimension for this fixed point is no longer 4 but $\tilde{d}_{c}=2$ and that the actual expansion parameter is

$$
\tilde{\epsilon}=2-d .
$$

In $d=3$, which is the case of interest for us, the dimension of $q$ is negative, which is equivalent to say that the only stable fixed point is $q^{*}=0$ (this can also be seen explicitly by writing the RG recursive equations for $q_{l}$ ). Therefore, the self-energy contribution in (83) vanishes and the kinetic coefficient has no perturbative contributions (at one loop), thus giving

$$
\Gamma_{l+1}=b^{z-2} \Gamma_{l},
$$

so that the only way to keep finite the kinetic coefficient at its fixed point is to have

$$
z=2,
$$

in agreement with the previous result. In this regime, $\psi$ behaves dynamically as an independent field; i.e., its relaxation has no contributions from the mode-coupling terms in the equations of motion. This highly nontrivial crossover between two different upper critical dimensions will be made more explicit in the Callan-Symanzik approach, which we describe the following section.

\section{CALLAN-SYMANZIK APPROACH}

In this section, we derive the RG results within a different renormalization approach, in which the large-scale properties of the system are deduced from a differential equation (called the Callan-Symanzik equation or renormalization group equation). This equation in turn follows, as we explain below, from a reparametrization invariance of the renormalized dynamic theory, which is introduced to deal with the strong cutoff $(\Lambda)$ dependence of the original theory (which leads to divergences in the $\Lambda \rightarrow \infty$ limit). This approach is complementary to the momentum-shell renormalization developed in Sec. IV. Its principles are described in several texts, e.g., Refs. [41,5052]. Our treatment of the ISM under the Callan-Symanzik (CS) approach follows the lines of the dynamic renormalization study of model E by De Dominicis and Peliti [44] (see also Ref. [52]). The CS approach involves the following steps:

(1) Write a renormalized theory, i.e., reparametrize the original dynamic functional in a way that all $\Lambda$ dependence (equivalently, divergencies that appear for $\Lambda \rightarrow \infty$ ) of physical observables is absorbed into a finite set of constants. This is done at an arbitrary momentum scale $\boldsymbol{\mu}$.

(2) Using the fact that the renormalization can be done at arbitrary values of $\boldsymbol{\mu}$, write a differential equation describing how relevant renormalized observables (in our case the response and correlation functions) change as $\mu=|\boldsymbol{\mu}|$ is varied. This is the RG equation, sometimes called the CS equation. Combining this with dimensional analysis, one finally obtains a differential equation that describes the change of the renormalized observables as the observation scale (external momentum) is varied. The coefficients of this equation are the $\beta$ functions, which are computed from the renormalization constants at a given order in perturbation theory. The equation is solved by the standard method of characteristics. 
(3) The solution by the method of characteristics shows that the behavior of the response and correlation functions at large scales can be obtained by studying the response and correlation at a fixed reference momentum scale but with observation-scale-dependent coupling constants. How the coupling constants change when increasing the observation scale is ruled by the $\beta$ functions, and the trajectories in parameter space induced by a change of scale are called $R G$ flow. Thus, one finally studies the RG flow, with particular attention to fixed points, which will lead to scaling behavior of the response.

We describe these steps in the following subsections. Many aspects of the calculation are identical to models $E$ and $G$, and for these we refer to the article by De Dominicis and Peliti [44]. We only describe in detail the aspects that are novel in the ISM.

\section{A. Renormalized theory and renormalization factors}

The diagrammatic expansion of the dynamical action involves integrals in momentum space that are divergent (in the space dimension of interest) for large integration momenta (ultraviolet divergences) unless some regularization procedure is adopted (like the cutoff $\Lambda$ for large momentum we used in the momentum-shell calculation; see Appendix A). To construct a renormalized theory means to reparameterize the functional in terms of a different set of coupling constants and fields in such a way that the divergences (or equivalently the details of the regularization procedure) are confined in a finite set of constants.

Instead of using a cutoff, here we renormalize according to the dimensional regularization plus minimal subtraction prescription [52]: Diverging integrals are evaluated in a dimension low enough that they are convergent, then analytically extended to noninteger dimension. The original divergences then show up as poles in the dimension variable. The minimal subtraction procedure consists in introducing the renormalized parameters so that they absorb only those poles.

Renormalization thus starts with the identification of all the ultraviolet divergences of the theory and with the definition of the renormalized constants to absorb them. Looking at the perturbative expansion, we see that the introduction of $\eta_{0}$ leaves the free propagator of the $\psi$ field (23) unchanged with respect to the model $\mathrm{G}$ case, while in the free propagator of the $s$ field $G_{0, s}(k, \omega)$ a $k$-independent term is added, so that the $k \rightarrow \infty$ behavior of the free propagators is unchanged. Then, since the structure of the diagrams is identical to that of models $\mathrm{G}$ and $\mathrm{E}$ (because the interacting part is the same), the divergences in ISM arise in the same diagrams. Then, from Ref. [44], we know that the theory is renormalizable in $d=4$ (which is the upper critical dimension of the theory). The divergent diagrams relevant to the dynamic renormalization arise in the expansion of $G_{\psi}(k, \omega)$ and $G_{s}(k, \omega)$, in particular in the derivatives

$$
\frac{\partial G_{\psi}^{-1}}{\partial k^{2}}, \quad \frac{\partial G_{s}^{-1}}{\partial k^{2}} .
$$

Both divergences are logarithmic in $d=4$. There are two additional divergences in $G_{\psi}(k, \omega)$ that we do not need to consider. One is the quadratic divergence in $G_{\psi}^{-1}(k=0, \omega=$
0) that is absorbed into a renormalized mass (susceptibility) in the static theory. Since we work here at the critical point defined by $r=0$, in practice this means setting $r_{0}=0$ in all the diagrams we consider. There is also a logarithmic divergence in $\partial G_{\psi}^{-1}(k, \omega) / \partial \omega$ that, however, does not arise at the one-loop level (and which is related to field renormalization).

The divergences are taken care in the following way: We consider the relevant divergent quantities [e.g., the derivatives in Eq. (87)] and evaluate them at $\omega=0$ and at given value of the momentum $\mathbf{k}=\boldsymbol{\mu}$ (so as to eliminate infrared divergences). We then replace the original kinetic and transport coefficients and coupling constants by renormalized counterparts that absorb the divergences, in a such a way that-once expressed in terms of the new parameters - the quantities of interest are finite. The renormalized parameters are defined through multiplication by $Z$ factors; when considering the derivatives in (87), this amounts to introducing renormalized kinetic coefficients

$$
\Gamma=Z_{\Gamma} \Gamma_{0}, \quad \lambda=Z_{\lambda} \lambda_{0} .
$$

The two remaining dynamic couplings, $g_{0}$ and $\eta_{0}$, do not pick up perturbative renormalization. In the case of $g_{0}$, this is a consequence of a Ward identity deriving from the fact that $\boldsymbol{s}$ generates the rotations of $\boldsymbol{\psi}$ (Appendixes B and C) [44]. In the case of $\eta_{0}$, the reason is that it is not involved in absorbing divergences due to the fact that $G_{s}^{-1}(k=0, \omega=0)$ is finite (see next section). We introduce, however, $\eta$ and $g$ as nondimensional counterparts of $\eta_{0}$ and $g_{0}$,

$$
g^{2}=K_{d} \mu^{d-4} g_{0}^{2}, \quad \eta=\frac{\eta_{0}}{\mu^{2}},
$$

where $K_{d}=2 \pi^{d / 2}(2 \pi)^{-d} / \Gamma(d / 2)$ is introduced for convenience and $\mu$ is the arbitrary momentum scale used to evaluate the propagators during renormalization. (Note that in this section we choose the frequency units so that $\Gamma_{0}$ and $\lambda_{0}$ are nondimensional, i.e., $[\omega]=\left[k^{2}\right]$ ).

The $Z$ factors now have to be determined at a given order in perturbation theory so that all the renormalized propagators (and in consequence correlation and response functions) are finite, i.e., the $Z$ factors are divergent in a such way that all observable quantities (expressed as averages with the renormalized theory) are finite. One can then in principle determine all the renormalized parameters of the model in terms of a finite number of observations (at fixed wave number and frequency). Finally, since at one loop, as mentioned above, the fields are not renormalized, the relation between original and renormalized propagators and correlations is

$$
\begin{gathered}
G_{\psi}\left(k, \omega, u_{0}, g_{0}, \Gamma_{0}, \lambda_{0}, \eta_{0}\right)=G_{\psi}^{R}(k, \omega, u, g, \Gamma, \lambda, \eta ; \mu), \\
C_{\psi}\left(k, \omega, u_{0}, g_{0}, \Gamma_{0}, \lambda_{0}, \eta_{0}\right)=C_{\psi}^{R}(k, \omega, u, g, \Gamma, \lambda, \eta ; \mu) .
\end{gathered}
$$

We discuss the determination of the $Z$ factors in below. These play a leading role in the CS procedure since they give the nontrivial contributions to the RG equation coefficients (Sec. VI B). 


\section{B. RG equation and dynamic critical exponent}

In this approach, the dynamic critical exponent is identified after finding the dynamic scaling form of the correlation functions. The procedure is the standard one, which we briefly recall. From the perturbation expansion at $\omega \neq 0$, one can see that the correlation function $C_{\psi}$ can be written in terms of the static coupling $u_{0}$, the effective dynamic couplings $\left(f_{0}, w_{0}\right.$, $\left.\mathcal{R}_{0}\right)$ and $\Gamma_{0}$, where $\Gamma_{0}$ and $\omega$ always appear in the combination $\omega / \Gamma_{0}$. So, we can rewrite Eq. (91) as

$$
C_{\psi}\left(k, \omega, u_{0}, g_{0}, \Gamma_{0}, \lambda_{0}, \eta_{0}\right)=C_{\psi}^{R}(k, \omega / \Gamma, u, f, w, \mathcal{R} ; \mu) .
$$

Since the left-hand side is independent of the arbitrary scale $\mu$, deriving with respect to $\log \mu$ one obtains the $\mathrm{RG}$ equation

$$
\left\{\mu \frac{\partial}{\partial \mu}+\sum_{l} \beta_{l} \frac{\partial}{\partial l}+v_{\Gamma} \Gamma \frac{\partial}{\partial \Gamma}\right\} C_{\psi}^{R}=0
$$

where $l=u, f, w, \mathcal{R}$ and the $\beta$ and $v$ functions are

$$
\beta_{l}(u, f, w, \mathcal{R})=\mu \frac{\partial l}{\partial \mu}, \quad v_{X}=\mu \frac{\partial \log Z_{X}}{\partial \mu} .
$$

The only dimensional arguments are $k, \omega$, and $\mu$, and the dimension of $C_{\psi}^{R}$ is 2 . Then dimensional analysis leads to an Euler equation, which can be used to eliminate the $\mu$ derivative:

$$
\left[\mu \frac{\partial}{\partial \mu}+\mathbf{k} \cdot \nabla_{k}+2 \omega \frac{\partial}{\partial \omega}\right] C_{\psi}^{R}=-2 C_{\psi}^{R} .
$$

Restricting ourselves to changes in the scale of $k$, i.e., $\mathbf{k}=$ $\boldsymbol{\mu} / b$, we have that $\mathbf{k} \cdot \nabla_{k}=-b \partial / \partial b$. Then, combining (93) and (95) we get

$$
\left\{\sum_{l} \beta_{l} \frac{\partial}{\partial l}+v_{\Gamma} \Gamma \frac{\partial}{\partial \Gamma}-2-2 \omega \frac{\partial}{\partial \omega}+b \frac{\partial}{\partial b}\right\} C_{\psi}^{R}=0
$$

(we have omitted terms that only appear beyond one loop). We solve (96) using as initial condition $b=1$, i.e., the value of the correlation at a reference $\mathbf{k}=\boldsymbol{\mu}$, at some frequency $\omega$, and at the physical values of the couplings $\Gamma, u, f, w$, and $\mathcal{R}$. The solution, found by the method of characteristics, is

$$
\begin{aligned}
& C_{\psi}^{R}\left(\frac{\boldsymbol{\mu}}{b}, \omega, \Gamma, u, f, w, \mathcal{R}\right) \\
& \quad=b^{2} C_{\psi}^{R}\left(\mathbf{k}_{0}, \frac{\omega b^{2}}{\hat{\Gamma}(b)}, \hat{u}(b), \hat{f}(b), \hat{w}(b), \hat{\mathcal{R}}(b)\right),
\end{aligned}
$$

with

$$
\hat{\Gamma}(b)=\Gamma \exp \left[-\int_{1}^{b} \frac{v_{\Gamma}\left(b^{\prime}\right)}{b^{\prime}} d b^{\prime}\right]
$$

and where $v_{\Gamma}$ depends on $b$ through the couplings $f, w, \mathcal{R}$. The dependence of these on $b$ is given by the functions $\hat{u}(b)$, etc. (the running coupling constants), which are the solution of the system

$$
\begin{aligned}
& b \frac{d \hat{u}}{d b}=-\beta_{u}(\hat{u}), \hat{u}(1)=u, \\
& b \frac{d \hat{f}}{d b}=-\beta_{f}(\hat{f}, \hat{w}, \hat{\mathcal{R}}), \hat{f}(1)=f,
\end{aligned}
$$

$$
\begin{aligned}
& b \frac{d \hat{w}}{d b}=-\beta_{w}(\hat{f}, \hat{w}, \hat{\mathcal{R}}), \hat{w}(1)=w, \\
& b \frac{d \hat{\mathcal{R}}}{d b}=-\beta_{\mathcal{R}}(\hat{\mathcal{R}}, \hat{f}), \hat{\mathcal{R}}(1)=\mathcal{R},
\end{aligned}
$$

where the $\beta$ functions must be computed perturbatively from the relation between the bare and renormalized couplings (88). At one loop, the flow of the static coupling constant $u$ is completely uncoupled from the dynamic couplings, so we do not take it into account in the following.

The meaning of (97) is that the correlation function at the physical values of the couplings $\vec{u} \equiv(u, f, w, \mathcal{R})$ and at a rescaled wave vector $\mu / b$ is equal to the correlation function at the original scale $\boldsymbol{\mu}$ but evaluated for different couplings $\hat{\vec{u}}(b)$. Fixed points thus are sets of coupling values $\vec{u}^{*}$ such that all $\beta$ functions vanish simultaneously: It is clear that if the flow starts at such a point, or approaches it for some large value of $b$, it will stay there for all larger $b$. If in addition the function $C_{\psi}^{R}(k, \omega / \Gamma, \vec{u})$ is continuous at $\vec{u}=\vec{u}^{*}$, then all the $k$ dependence at large $b$ (small $k$ ) is contained in $\omega b^{2} / \Gamma(b)$ : Equation (97) is then the scaling law we seek, and we can read off the scaling behavior from its second argument even if we do not know the form of $C_{\psi}^{R}$. For example, if the flow is near a fixed point for $b>b^{*}$, then $v_{\Gamma}(b)=v_{\Gamma}(\vec{u}(b)) \approx v_{\Gamma}\left(\vec{u}^{*}\right) \equiv v_{\Gamma}^{*}$. Then, (98) gives

$$
\hat{\Gamma}(b) \approx \Gamma \exp \left[-\int_{1}^{b^{*}} \frac{v_{\Gamma}\left(b^{\prime}\right)}{b^{\prime}} d b^{\prime}-v_{\Gamma}^{*} \log \left(b / b^{*}\right)\right] \sim b^{-v_{\Gamma}^{*}} .
$$

Since $k=\mu / b$, we have $b \sim k^{-1}$ and

$$
\frac{\omega b^{2}}{\hat{\Gamma}(b)} \sim \omega b^{2+v_{\Gamma}^{*}} \sim \omega k^{-z}, \quad z=2+v_{\Gamma}^{*} ;
$$

i.e., the value of $v_{\Gamma}$ at the fixed point gives the correction to the naive dynamic critical exponent.

So we proceed next (Secs. VIC and VID) to determine the $Z$ factors that furnish the $\beta$ functions, and then (Sec. VIE) to find the fixed points of the flow (99) and their infrared (i.e., $b \rightarrow \infty)$ stability. The infrared stable fixed points will rule the scaling behavior at large length scales. Unstable fixed points may, depending on initial conditions, lead to transient scaling laws observable in certain regimes.

\section{Determination of $\boldsymbol{Z}$ factors}

We must determine the two dynamic $Z$ factors $Z_{\lambda}$ and $Z_{\Gamma}$ (in a two-loop or higher calculation, a third factor related to field renormalization would arise, but we do not need it here). First, $Z_{\lambda}$ is fixed by requiring that $\partial G_{S}^{-1} /\left.\partial k^{2}\right|_{\omega=0, k=\mu}$ be finite. We have

$$
G_{s}^{-1}(k, \omega)=\left[-i \omega+\eta_{0}+\lambda_{0} k^{2}-\Pi(k, \omega)\right],
$$

where $\Pi$ is the same self-energy as in Eq. (32), although from now on all the integrals in $k$ in the self-energies will no longer be performed on shell but rather between 0 and $\infty$, and for this reason we drop the subscript $b$ from the self-energy symbols. From Eq. (90), we then have

$$
\left.\frac{\partial\left(G_{s}^{R}\right)^{-1}}{\partial k^{2}}\right|_{\substack{k=\mu \\ \omega=0}}=\frac{\lambda}{Z_{\lambda}}-\left.\frac{\partial \Pi}{\partial k^{2}}\right|_{\substack{k=\mu \\ \omega=0}} .
$$


From this equation, two conclusions follow: The first is that the $\lambda_{0} k^{2}$ term cannot be left out from a renormalizable theory. This is a consequence of the fact that, in an expansion of $\Pi$ in the external wave vector, it is the $k^{2}$ coefficient that is divergent, not that of $k^{0}$ (in fact $\Pi(k=0)=0$ ). Thus, if $\lambda_{0}$ is absent, it is useless to define $\eta=Z_{\eta} \eta_{0}$ and try to absorb the pole of $\Pi$ into $Z_{\eta}$, because $\eta$ drops out from (102). This is equivalent to the finding, in the momentum-shell scheme, that the renormalization transformation generates a $\lambda$ coefficient even if it is absent in the original theory.

The second conclusion is that $Z_{\lambda}$ is determined solely by the behavior of $\Pi$, and, since at one loop this self-energy is unchanged with respect to model $\mathrm{G}$, we can without further discussion write it from the model $G$ result [44]:

$$
Z_{\lambda}=1+\frac{f}{2 \epsilon} \text {. }
$$

At one loop, the differences between model G and ISM are only found in $Z_{\Gamma}$, which we proceed to compute now. The propagator of the $\psi$ field is

$$
G_{\psi}^{-1}(k, \omega)=\delta_{\alpha \beta}\left[-i \omega+\Gamma_{0}\left(k^{2}+r_{0}\right)-\Sigma(k, \omega)\right],
$$

where $\Sigma$ is the self-energy (31), which we recall here for convenience:

$$
\begin{aligned}
\Sigma= & -2 g_{0}^{2} \int \frac{d^{d} p}{(2 \pi)^{d}} \frac{k^{2}+r_{0}}{p^{2}+r_{0}} \\
& \times \frac{1}{-i \omega+\Gamma_{0}\left(p^{2}+r_{0}\right)+\lambda_{0}(\mathbf{k}-\mathbf{p})^{2}+\eta_{0}} .
\end{aligned}
$$

We must now consider the renormalized derivative

$$
\left.\frac{\partial\left(G_{\psi}^{R}\right)^{-1}}{\partial k^{2}}\right|_{\substack{k=\mu \\ \omega=0}}=\frac{\Gamma}{Z_{\Gamma}}-\left.\frac{\partial \Sigma}{\partial k^{2}}\right|_{\substack{k=\mu \\ \omega=0}}
$$

and choose $Z_{\Gamma}$ so that it is finite. In the dimensional regularization procedure, this means that the $Z$ factor cancels the poles that appear for $d=4$ (i.e., terms proportional to $1 / \epsilon$, $\epsilon=4-d)$, so that (106) is free of poles. Computing the derivative from (105) for general dimension $d=4-\epsilon$ at the critical point and ignoring the contribution from a convergent integral, one has

$$
\begin{aligned}
\left.\frac{\partial\left(G_{\psi}^{R}\right)^{-1}}{\partial k^{2}}\right|_{\substack{k=\mu \\
\omega=0}} & =\Gamma_{0}\left\{1+K_{d} \frac{\Gamma(d / 2)}{\pi^{d / 2} \Gamma_{0}} \frac{g_{0}^{2} \mu^{d-4}}{\Gamma_{0}+\lambda_{0}} \int \frac{d^{d} x}{x^{2}} \frac{1}{x^{2}+\left[-2 \lambda_{0} \hat{\boldsymbol{\mu}} \cdot \mathbf{x}+\lambda_{0}+\eta_{0} / \mu^{2}\right] /\left[\Gamma_{0}+\lambda_{0}\right]}\right\} \\
& =\Gamma_{0}\left\{1+K_{d} \Gamma(d / 2) \frac{g_{0}^{2}}{\Gamma_{0} \lambda_{0}} \frac{\mu^{-\epsilon}}{1+\Gamma_{0} / \lambda_{0}} \Gamma(2-d / 2) I_{\psi}(\mathcal{R}, w)\right\},
\end{aligned}
$$

where $\mathcal{R}^{2}=\lambda / \eta=\mu^{2} Z_{\lambda} \lambda_{0} / \eta_{0}, \hat{\boldsymbol{\mu}}=\boldsymbol{\mu} / \mu$, and

$$
I_{\psi}(\mathcal{R}, w) \equiv \int_{0}^{1} d \beta\left[\frac{1+\mathcal{R}^{-2}}{1+w} \beta-\frac{1}{(1+w)^{2}} \beta^{2}\right]^{d / 2-2}
$$

The renormalized counterpart can therefore be written as

$$
\left.\frac{\partial\left(G_{\psi}^{R}\right)^{-1}}{\partial k^{2}}\right|_{\substack{k=\mu \\ \omega=0}}=\Gamma\left\{\frac{1}{Z_{\Gamma}}+\frac{f}{1+w} \Gamma(d / 2) \Gamma(2-d / 2) I_{\psi}(\mathcal{R}, w)\right\},
$$

The one-loop term has a pole in $d=4$ (from the second $\Gamma$ function): This is how the original divergence of the integral in $d=4$ manifests itself in dimensional regularization. The minimal renormalization prescription stipulates that this pole be identified so that an equivalent pole but with opposite residue can be added to $1 / Z_{\Gamma}$, thus making the renormalized vertex finite. So we expand the second term: Setting $\mathcal{I}(\mathcal{R}, w)=\left(1+\mathcal{R}^{-2}\right) \beta /(1+w)-\beta^{2} /(1+w)^{2}$,

$$
\begin{aligned}
\frac{\partial\left(G_{\psi}^{R}\right)^{-1}}{\partial k^{2}}=\Gamma & \left\{\frac{1}{Z_{\Gamma}}+\frac{f}{1+w}\left[\frac{2}{\epsilon}+O\left(\epsilon^{0}\right)\right]\right. \\
& \left.\times\left[1-\frac{\epsilon}{2} \int_{0}^{1} d \beta \log \mathcal{I}(\mathcal{R}, w)+O\left(\epsilon^{2}\right)\right]\right\} \\
= & \Gamma\left\{\frac{1}{Z_{\Gamma}}+\frac{2}{\epsilon} \frac{f}{1+w}+\cdots\right\} .
\end{aligned}
$$

As long as $\mathcal{R} \neq 0$, all singular behavior is contained in the pole at $d=4$, i.e., the term proportional to $1 / \epsilon$ in the last line of (110). Then, defining $Z_{\Gamma}=1+(2 / \epsilon) f /(1+w)$ renders $G_{\psi}^{R}$ finite. Thus, naively one finds that $Z_{\Gamma}$ is independent of $\mathcal{R}$, and, since $Z_{\lambda}$ is also independent of $\mathcal{R}$ at one loop, this leads to $\beta$ functions for the parameters $f$ and $w$ that are independent of $\mathcal{R}$ and thus to flow equations identical to model $\mathrm{G}$ for $f$ and $w$, uncoupled to the flow of $\mathcal{R}$. However, this is wrong: We have already seen, in the momentum-shell scheme, that the presence of $\eta_{0}$ profoundly affects the flow of $f$ and $w$, with the notable macroscopic consequence of a change in the dynamic critical exponent.

Even without the insight we have from the momentumshell calculation, one could guess that the naive expectation cannot be right: Since $\mathcal{R}_{0}$ has the dimensions of a length, one expects that its stable fixed point is 0 , and indeed below we shall find from the $\beta$ function (118) that $\mathcal{R} \sim b^{-1+f^{*} / 4}$ for $b \rightarrow \infty$, with $f^{*}$ of order $\epsilon$. In the above equations, one sees that the limit $\mathcal{R} \rightarrow 0$ requires special treatment: In (110), a logarithmic divergence appears in the $\epsilon$ expansion of $I(\mathcal{R}, w)$, and even before expanding one sees that (108) is problematic because $I_{\psi}(\mathcal{R} \rightarrow 0, w)$ vanishes for $d<4$, while $\lim _{\mathcal{R} \rightarrow 0} \lim _{d \rightarrow 4} I_{\psi}(\mathcal{R}, w)=1$.

The difficulty here is that the length scale $\mathcal{R}$ occurs in the Gaussian part of the dynamical functional and that it appears in the loop integrals in such a way that their convergence properties change at one of the fixed points of $\mathcal{R}$. To deal with this, we use a generalized minimal substraction as discussed by Frey and others $[53,54]$. This method involves incorporating 
the singular $\mathcal{R}$ dependence into the renormalization $Z$ factors (and consequently into the $\beta$ functions): We thus "enrich" the pole with a crossover factor $X(\mathcal{R}, w)$ extracted from $I(\mathcal{R}, w)$ which absorbs the singular $\mathcal{R} \rightarrow 0$ behavior. Let us rewrite (109) as

$$
\begin{aligned}
\frac{\partial\left(G_{\psi}^{R}\right)^{-1}}{k^{2}}= & \Gamma\left\{\frac{1}{Z_{\Gamma}}+\frac{f}{1+w} \Gamma(d / 2) \Gamma(2-d / 2)\right. \\
& \left.\times X(\mathcal{R}, w) X^{-1}(\mathcal{R}, w) I_{\psi}(\mathcal{R}, w)\right\},
\end{aligned}
$$

where $X(\mathcal{R}, w)$ is such that $X^{-1}(\mathcal{R}, w) I_{\psi}(\mathcal{R}, w)$ (and in consequence all coefficients of its $\epsilon$ expansion) is well behaved for all values of $\mathcal{R}$ (including $\mathcal{R}=0, \mathcal{R}=\infty$ ). We discuss in the next subsection how to fix this factor, but before let us write the renormalization factors including the as yet unknown $X(\mathcal{R}, w)$ :

$$
\begin{gathered}
Z_{\lambda}=1+\frac{1}{\epsilon} f / 2, \\
Z_{\Gamma}=1+\frac{1}{\epsilon} 2 X(\mathcal{R}, w) \frac{f}{1+w} .
\end{gathered}
$$

We conclude this subsection writing $v_{\Gamma}, v_{\lambda}$, and the $\beta$ functions for the couplings $f, w$ and for $\mathcal{R}$. These functions determine the RG flow and the asymptotic scaling properties of the observables (Sec. VIB). Recalling the definition (94) of $\beta$ functions and $v$ exponents, and developing them to first order in $f$, we get

$$
\begin{gathered}
v_{\Gamma}=-2 X(\mathcal{R}, w) \frac{f}{1+w}, \\
v_{\lambda}=-\frac{f}{2},
\end{gathered}
$$

so that the $\beta$ functions [to be compared to (60)] are the following:

$$
\begin{gathered}
\beta_{f} \equiv \mu \frac{\partial f}{\partial \mu}=-f\left(\epsilon+v_{\Gamma}+v_{\lambda}\right) \\
=-f\left[\epsilon-f\left(\frac{1}{2}+\frac{2 X}{1+w}\right)\right], \\
\beta_{w} \equiv \mu \frac{\partial w}{\partial \mu}=w\left(v_{\Gamma}-v_{\lambda}\right)=w f\left[\frac{1}{2}-\frac{2 X}{1+w}\right], \\
\beta_{\mathcal{R}} \equiv \mu \frac{\partial \mathcal{R}}{\partial \mu}=\mathcal{R}\left(1+v_{\lambda} / 2\right)=\mathcal{R}\left[1-\frac{1}{4} f\right] .
\end{gathered}
$$

\section{Determination of the crossover factor $X$}

To determine $X(\mathcal{R}, w)$, the idea is to to fix it in such a way that the renormalization factor $Z_{\Gamma}$ contains all the singularities near both fixed points $\mathcal{R}=0$ and $\mathcal{R}=\infty$. When $\mathcal{R}$ is nonzero, the only singularity in (109) is the pole at $d=4$ originating in the $\Gamma$ function $\Gamma(2-d / 2)$. Thus, the first condition we impose is that

$$
\lim _{\mathcal{R} \rightarrow \infty} X(\mathcal{R}, w)=1 .
$$

To find another condition, we must study $G_{\psi}^{-1}$ for vanishing $\mathcal{R}$. To do this, let us define a new parameter

$$
q \equiv \frac{g^{2}}{\Gamma \eta}=\frac{K_{d} g_{0}^{2} \mu^{d-4}}{Z_{\Gamma} \Gamma_{0} \mu^{-2} \eta_{0}}=f \mathcal{R}^{2},
$$

and rewrite (107) as

$$
\begin{aligned}
\frac{\partial\left(G_{\psi}^{R}\right)^{-1}}{\partial k^{2}}= & \Gamma\left\{\frac{1}{Z_{\Gamma}}+q \frac{\Gamma(d / 2)}{\pi^{d / 2}} \int \frac{d^{d} x}{x^{2}+m^{2}}\right. \\
& \left.\times \frac{1}{\mathcal{R}^{2} w x^{2}+\mathcal{R}^{2}(\mathbf{x}-\hat{\boldsymbol{\mu}})^{2}+1}\right\},
\end{aligned}
$$

where we have introduced a constant $m^{2}$ to avoid an infrared divergence in $d=2$. We can now set $\mathcal{R}=0$ in the integral to find

$$
\frac{\partial\left(G_{\psi}^{R}\right)^{-1}}{\partial k^{2}}=\Gamma\left\{\frac{1}{Z_{\Gamma}}+q \Gamma(1-d / 2) \Gamma(d / 2) m^{d-2}\right\} .
$$

We now find a pole at $d=2$, corresponding to the fact that for $\mathcal{R}=0$ the integral in (121) diverges for $d \geqslant 2$ : The critical dimension for $q$ is $d_{c}=2$, not 4 . We then expand around $d_{c}=2$ and find

$$
\begin{gathered}
Z_{\Gamma}=1+q \frac{2}{2-d}=1+\frac{2}{\tilde{\epsilon}} q, \\
\beta_{q}=-(2-d) q+2 q^{2}=-\tilde{\epsilon} q+2 q^{2},
\end{gathered}
$$

where

$$
\tilde{\epsilon}=2-d .
$$

We note that $\beta_{q}$ above is correct up to second order in $\tilde{\epsilon}$, i.e., around the new critical dimension $d_{c}=2$. Equation (124) can give us the condition to impose on $X(\mathcal{R}, w)$ near the other fixed point: From (116) and (118), we can write

$$
\beta_{q}=\mathcal{R}^{2} \beta_{f}+2 \mathcal{R} f \beta_{\mathcal{R}}=-q\left[2-d-2 X(\mathcal{R}, w) \frac{f}{1+w}\right],
$$

so that to recover (124) we impose

$$
\lim _{\mathcal{R} \rightarrow 0} \lim _{d \rightarrow 2} X(\mathcal{R}, w)=(1+w) \mathcal{R}^{2} .
$$

A simple choice for $X(\mathcal{R}, w)$ is then

$$
X(\mathcal{R}, w)=\left[\frac{(1+w) \mathcal{R}^{2}}{1+(1+w) \mathcal{R}^{2}}\right]^{a(d)}
$$

with $a(d=2)=1$. Referring to (111), we find

$$
\begin{aligned}
X^{-1}(\mathcal{R}, w) I_{\psi}(\mathcal{R}, w) & =\int_{0}^{1} d \beta\left[\frac{(1+w) \mathcal{R}^{2}}{1+(1+w) \mathcal{R}^{2}}\right]^{-a}\left[\frac{1+1 / \mathcal{R}^{2}}{1+w} \beta-\frac{1}{(1+w)^{2}} \beta^{2}\right]^{-\epsilon / 2} \\
& =\int_{0}^{1} d \beta\left[\frac{(1+w) \mathcal{R}^{2}}{1+(1+w) \mathcal{R}^{2}}\right]^{-a+\epsilon / 2}\left[\frac{1+\mathcal{R}^{2}}{1+(1+w) \mathcal{R}^{2}} \beta-\frac{\mathcal{R}^{2}}{1+(1+w) \mathcal{R}^{2}} \frac{\beta^{2}}{1+w}\right]^{-\epsilon / 2}
\end{aligned}
$$


so that $X(\mathcal{R}, w)$ defined as in (128) with $a=\epsilon / 2$ fulfills the three conditions: (i) $X(\mathcal{R} \rightarrow \infty)=1$, (ii) $X(\mathcal{R} \rightarrow 0, d \rightarrow$ $2)=(1+w) \mathcal{R}^{2}$, and (iii) $X^{-1}(y, w) I(y, w)$ is finite and nonvanishing for all values of $\mathcal{R}$, so that all terms not included in $Z_{\Gamma}$ (i.e., terms of order $\epsilon^{0}$ or higher) are regular and do not cause further trouble. In particular, $\lim _{\mathcal{R} \rightarrow 0} \lim _{d \rightarrow 4} X^{-1} I_{\psi}=$ $\lim _{d \rightarrow 4} \lim _{\mathcal{R} \rightarrow 0} X^{-1} I_{\psi}=1$.

A subtle but important point, however, remains to be made. At face value, our choice of $X(\mathcal{R}, w)$ recovers the flow (124) only at $d$ exactly equal to 2 , while (124) has actually been obtained in a general dimension. Thus, it seems that one would want $a=1$, which, however, accounts for the $\mathcal{R} \rightarrow$ 0 behavior of $I(\mathcal{R}, w)$ only at $d=2$. The way out of this seeming inconsistence is to remember that (124) is valid in general dimension but only up to second order in $\tilde{\epsilon}=2-d=$ $\epsilon-2$. This means that one should actually write this exponent as $a=1+\tilde{\epsilon} / 2$, and (128) as

$$
\begin{gathered}
X(\mathcal{R}, w)=\frac{(1+w) \mathcal{R}^{2}}{1+(1+w) \mathcal{R}^{2}}\left\{1+\frac{\tilde{\epsilon}}{2} \log \left[\frac{(1+w) \mathcal{R}^{2}}{1+(1+w) \mathcal{R}^{2}}\right]\right. \\
+\cdots\} .
\end{gathered}
$$

It is then clear that (since the nonzero fixed point of $q$ will be of order $\tilde{\epsilon}$ ) (126) indeed recovers (124) in the limit $\mathcal{R} \rightarrow 0$ for general dimension up to order $\tilde{\epsilon}^{2}$. But then it also becomes clear that (124) cannot fix $X(\mathcal{R}, w)$ at order $\tilde{\epsilon}$ and beyond. So $a=1+\tilde{\epsilon} / 2$ is fine near $d=2$ and also near $d=4$ [where (109) must be expanded].

From these considerations, in what follows, we will simply set $a=1$ when writing the $\beta$ functions also in $d=3$, which is the dimension of interest here. We do so because this is the simplest choice that gives a correct description of the flow at the one-loop level: When $\mathcal{R} \rightarrow 0$ the $O(\tilde{\epsilon})$ contributions to $X(\mathcal{R}, w)$ cannot be fixed without going to two loops so we may as well omit them, and on the other hand near the $\mathcal{R} \rightarrow \infty$ fixed point $X(\mathcal{R}, w) \rightarrow 1$ independently of the exponent. So the final form of $\beta$ functions we find within the CS scheme is identical to Eq. (60) we previously obtained with the momentum-shell technique.

\section{E. Fixed points}

Equations (118) and (99d) show that $\mathcal{R}^{*}=0$ and $\mathcal{R}^{*}=\infty$ are fixed points of $\mathcal{R}$. The flow can be solved formally as

$$
\hat{\mathcal{R}}(b)=\mathcal{R} \exp \left[-\int_{1}^{b} \frac{1-f\left(b^{\prime}\right) / 4}{b^{\prime}} d b^{\prime}\right] .
$$

Since we expect (and confirm below) that the fixed point of $f$ will be of order $\epsilon$, we see that the integrand within the exponential is negative (for large $b$ ) at least, and that $\mathcal{R}^{*}=0$ is IR stable while $\mathcal{R}^{*}=\infty$ is IR unstable.

\section{1. $\mathcal{R}^{*}=\infty$-conservative fixed point}

The $\mathcal{R}^{*}=\infty$ fixed point corresponds to $X(\mathcal{R}, w)=1$. Since it is unstable, it is only relevant for $b \rightarrow \infty$ when the system starts at $1 / \mathcal{R}=0$. This corresponds to a very important special case, namely $\eta_{0}=0$, i.e., model $\mathrm{G}$ (equivalent to model $\mathrm{E}$ for what concerns the scaling properties). It is also relevant at moderately large scales for very small $1 / \mathcal{R}$, when the flow stays near $X=1$ long enough that the other couplings approach the model $\mathrm{E}$ fixed point before $\mathcal{R}$ becomes so small that $X$ is significantly different from 1 (see Secs. VD and VIF).

Equations (99b) and (99c) for $X=1$ were studied by DeDominics and Peliti [44], who considered model E at two loops, and we refer to them for the analysis of the fixed points and their stability. In summary, the relevant [IR stable in the $(f, w)$ subspace] fixed point is

$$
f^{*}=\epsilon, \quad w^{*}=3, \quad \mathcal{R}^{*}=\infty,
$$

implying

$$
v_{\Gamma}^{*}=-\frac{\epsilon}{2}, \quad z=2-\frac{\epsilon}{2}=\frac{d}{2} .
$$

It is interesting to remark that the result for $z$, although obtained here at one loop, has to be valid at all orders in perturbation theory as long as $w^{*}$ and $f^{*}$ are different from 0 : If $w$ is non-null, (117) implies $v_{\Gamma}=v_{\lambda}$, and (116) then gives $2 v_{\Gamma}=-\epsilon$.

\section{2. $\mathcal{R}^{*}=0$ - dissipative fixed point}

When $\mathcal{R}=0, X(\mathcal{R}, w)=0$, which gives immediately

$$
v_{\Gamma}^{*}=0, \quad z=2,
$$

regardless of $w$ and $f$ (as long as they are finite). Setting $X(\mathcal{R}, w)=0$ in (99b) and (99c), one finds two solutions:

$$
f^{*}=0
$$

and

$$
f^{*}=2 \epsilon, \quad w^{*}=0 .
$$

The stability of the two fixed points can be studied linearizing the flow (99) around the fixed point $\vec{u}^{*}$ :

$$
b \frac{d \vec{u}}{d b}=-W\left(\vec{u}-\vec{u}^{*}\right),
$$

where $W$ is the Jacobian matrix

$$
W=\left(\begin{array}{ccc}
\frac{\partial \beta_{f}}{\partial f} & \frac{\partial \beta_{f}}{\partial w} & \frac{\partial \beta_{f}}{\partial \mathcal{R}} \\
\frac{\partial \beta_{w}}{\partial f} & \frac{\partial \beta_{w}}{\partial w} & \frac{\partial \beta_{w}}{\partial \mathcal{R}} \\
\frac{\partial \beta_{\mathcal{R}}}{\partial f} & \frac{\partial \beta_{\mathcal{R}}}{\partial w} & \frac{\partial \beta_{\mathcal{R}}}{\partial \mathcal{R}}
\end{array}\right)
$$

evaluated at the fixed point. The fixed point is stable if $W$ is positive definite; i.e., its eigenvalues are all positive. We find for the two cases above

$$
W(f=0, \mathcal{R}=0)=\left(\begin{array}{ccc}
-\epsilon & 0 & 0 \\
1 / 2 & 0 & 0 \\
0 & 0 & 1
\end{array}\right)
$$

which has eigenvalues 0,1 , and $-\epsilon$, while

$W(f=2 \epsilon, w=0, \mathcal{R}=0)=\left(\begin{array}{ccc}\epsilon & 0 & 0 \\ 1 / 2 & \epsilon / 2 & 0 \\ 0 & 0 & 1-\epsilon\end{array}\right)$,

with eigenvalues $\epsilon, \epsilon / 2$, and $1-2 \epsilon$. So the only IR stable fixed point (at 1 loop, near $d=4$ ) is $f^{*}=2 \epsilon, w^{*}=0, \mathcal{R}^{*}=$ 0 , which implies as we have seen that the critical exponent is $z=2$. 


\section{F. Crossover}

We have just concluded that the only IR-stable fixed point gives $z=2$, so that for large observation scales $(k \rightarrow 0)$ the critical dynamics is like that of a purely dissipative model (like model A) when the starting (physical) value of $\eta$ is nonzero. However, for nonzero but small $\eta$, such that the starting $\mathcal{R}$ is very large, the initial value of $X(\mathcal{R}, w)$ is very close to 1 and will stay so until $\mathcal{R}(b)$ is of order 1 (e.g., for $w=3, X$ is larger than 0.99 for $\mathcal{R}>5$ ). So one can expect that $f$ and $w$ will at first move as if $X=1$, i.e., toward the conservative (model $\mathrm{G})$ fixed point, staying in its neighborhood until $\mathcal{R}$ decreases significantly, and in effect the numerical study of the flux (Fig. 1) confirms this expectation. Then, experimentally one will observe model $\mathrm{G}$ critical behavior $(z=d / 2)$ for moderate (i.e., not too small) values of $k$, possibly lasting a rather wide interval, until at some point for $k \rightarrow 0$ the asymptotic $z=2$ exponent will be seen. We show here how to obtain the scaling of the wave vector $k_{c}$ (marking the end of the model G behavior) with the physical value of $\mathcal{R}$ (cf. Sec. V E).

Assume then that the physical value of $\mathcal{R}$ is $\hat{\mathcal{R}}(b=1) \equiv$ $\mathcal{R}_{1} \gg 1$ so that $X\left(\mathcal{R}_{1}, w(1)\right) \approx 1$. Assume also that one is observing at a scale $k=\mu / b$ such that the flow has already reached the neighborhood of the $z=d / 2$ fixed point (which in particular implies $v_{\Gamma} \approx-\epsilon / 2$ ). We ask how small we must make $k$ so that the system moves away from this fixed point and the scaling law changes. For this to happen, $X$ must be significantly less than 1 , so let us impose that $X<c$, with $c=$ 0.99 say. This requires $\mathcal{R}(k)<\mathcal{R}_{c}=\sqrt{c /[4(1-c)]}$. Now since we are near the conservative fixed point, we can use (99d) with $v_{\Gamma}=v_{\Gamma}^{*}$ to obtain

$$
\mathcal{R}(k) \approx \mathcal{R}_{1}(k / \mu)^{1+v_{\lambda}^{*} / 2} .
$$

Now the crossover wave vector will be such that $\mathcal{R}\left(k_{c}\right)=\mathcal{R}_{c}$, so that

$$
k_{c} \sim \mathcal{R}_{1}^{-1 /\left(1+v_{\lambda}^{*} / 2\right)}=\mathcal{R}_{1}^{-1 /(1-\epsilon / 4)}=\mathcal{R}_{1}^{-4 / d} .
$$

Hence, we find the same crossover exponent as in momentum shell, namely,

$$
\kappa=4 / d .
$$

Let us notice that the crossover exponent $\kappa$ is nontrivial: From naive dimensional analysis, one would have guessed $k_{c} \sim \mathcal{R}_{1}^{-1}$. However, the renormalized $\mathcal{R}$ is dimensionless, and the RG result is actually taking into account the nontrivial effects of the hidden microscopic length scale.

Finally, let us note that the crossover exponent derives its value from $v_{\lambda}$ at the model $\mathrm{G}$ fixed point which, as we have mentioned before, takes the value $-\epsilon / 2$ at all orders in perturbation theory [44] and thus so must the crossover exponent.

\section{NUMERICAL SIMULATIONS}

To test our results, we performed numerical simulations of the microscopic ISM model on a fixed lattice in $d=$ 3. We implemented the dynamical equations (10) and (11) using a generalized Verlet integrator [55-57] for second-order equations. Details of the algorithm can be found in Ref. [58]. The lattice spacing is $\Lambda^{-1}=1$. We fixed the parameters $\hat{J}=1, \hat{\chi}=1$, and performed simulations at several values of the temperature $T$ and of the friction coefficient $\hat{\eta}$. Since the temperature sets the correlation length and the friction regulates the conservation length scale $\mathcal{R}_{0}$, we can in this way explore the $\left(\xi, \mathcal{R}_{0}\right)$ plane of Fig. 2 . For all values of $T$ and $\hat{\eta}$ considered, we computed the correlation length $\xi$ and the relaxation time $\tau$ and inferred the exponent $z$ from the scaling relation Eq. (8) between them. In this way, we could investigate the dynamical critical behavior and compare results with the predictions of the RG computation. Before illustrating the results, let us briefly explain the procedure followed to compute the main quantities required for our analysis, namely $\xi$ and $\tau$.

\section{A. Static behavior and determination of $\xi$}

Since we are interested in the critical behavior of the system, we need first of all to locate the transition temperature and characterize the static critical properties of the system. To do so, we perform numerical simulations of Eqs. (10) and (11) in the stationary regime and use decorrelated dynamical configurations to compute one-time equilibrium averages (from now on indicated with $\langle\cdots\rangle)$. From the static point of view, the ISM on a lattice is completely equivalent to a standard ferromagnetic model, we therefore expect static properties to reproduce the well-known results of the Heisenberg model. For a system of $N$ velocities and vectors, the polarization is defined as

$$
\boldsymbol{\Phi}=\frac{1}{N \psi_{0}} \sum_{i} \boldsymbol{\psi}_{i}
$$

and its modulus, the scalar polarization $\phi$. Here, $\psi_{0}$ is the modulus of the vectors $\boldsymbol{\psi}$ (which are all the same and equal to 1 in our case). $\boldsymbol{\Phi}$ measures the degree of global alignment. The average value of this quantity is plotted in Fig. 3(a) as a function of temperature, and clearly shows the occurrence of an ordering transition. The critical temperature can be conveniently located by looking at the fluctuations of $\langle\phi\rangle$, namely the susceptibility

$$
\chi=\beta N\left[\left\langle\phi^{2}\right\rangle-\langle\phi\rangle^{2}\right],
$$

where $\beta$ is the inverse of the temperature. We analyzed these quantities for a wide range of temperatures $(0.1 \leqslant T \leqslant 5.0)$ and sizes $(N=512,2197,4096,8000)$. From Figs. 3(a) and 3(b), we can see that the critical temperature is located approximately at $T_{c} \simeq 1.5$. As usual, finite-size effects imply that the critical point moves toward lower temperatures as the linear size of the system increases [59]. This happens because the correlation length $\xi$, and hence the susceptibility $\chi$, saturates close to the critical point so that the effective $T_{c}$ decreases as the linear size of the system increases [60].

To measure the correlation length $\xi$, we first computed the static connected correlation function $C(r)$ :

$$
C(r)=\frac{\sum_{i, j}\left\langle\delta \boldsymbol{\psi}_{i} \cdot \delta \boldsymbol{\psi}_{j}\right\rangle \delta\left(r-r_{i j}\right)}{\sum_{i, j} \delta\left(r-r_{i j}\right)},
$$

where $r_{i j}$ is the distance between two sites $i$ and $j$, and $\delta \psi_{i}=$ $\boldsymbol{\psi}_{i}-\left\langle\boldsymbol{\psi}_{i}\right\rangle$. Since we are mostly interested in the paramagnetic phase of the model, the relevant one to describe swarms, we focused on temperatures $T>T_{c}$, approaching the critical point from above. The behavior of the correlation function 

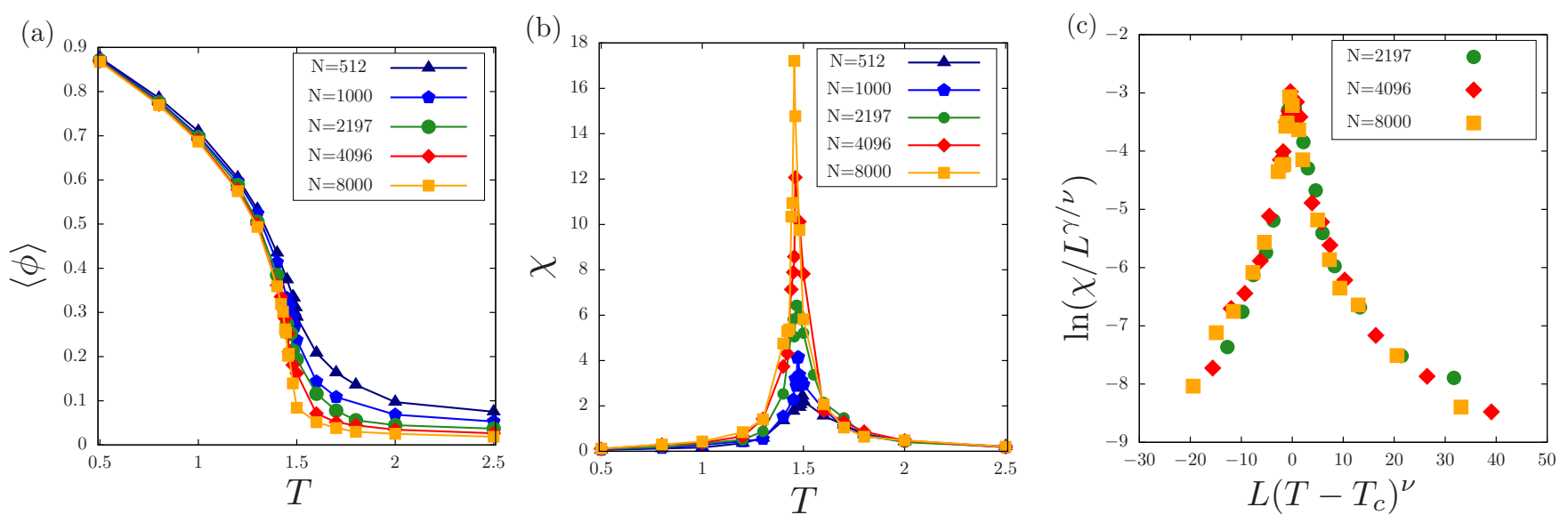

FIG. 3. Static critical behavior. (a) Average scalar polarization for temperatures $0.5 \leqslant T \leqslant 2.5$ and for different sizes $(N=512,1000$, 2197, 4096, 8000). An ordering transition occurs at approximately $T_{c} \simeq 1.5$. (b) Susceptibility as a function of temperature, same sizes as in panel (a); the maximum of each curve is located at a temperature that decreases with increasing the size of the system and approaches the critical temperature $T_{c}$ in the thermodynamic limit. (c) Finite-size scaling of the susceptibility. Curves at $N=2197,4096,8000$ satisfy finite-size scaling with exponents $v=0.707$ and $\gamma / v=1.973$, as predicted by the theory of the Heisenberg model [41].

(not displayed) is as expected for a Heisenberg model, and we therefore computed the correlation length from the expression $r C(r)=\exp (-r / \xi)$, exploiting the fact that the anomalous dimension is small [41]. We combined this information with data on the susceptibility to obtain an estimate of the ratio between critical exponents $\gamma / v$ for sizes $N=2197$, 4096, 8000. Simulations at $N=8000$ gave $\gamma / \nu=1.905$ in agreement with the literature [41]. We therefore used this size for all following analyses. Finally, to further test the equivalence of the static properties of ISM with the Heisenberg model we performed a finite-size scaling analysis on the susceptibility, as displayed in Fig. 3(c).

\section{B. Dynamic behavior and determination of $\tau$}

To investigate the dynamical behavior of the system one has to look at time-dependent quantities. In particular, the characteristic timescale $\tau$ is by definition the scale over which fluctuations of the order parameter become decorrelated. To compute it, we introduce the spatiotemporal correlation function, that is,

$$
\begin{aligned}
C(k, t) & =\frac{1}{N} \sum_{i, j} \frac{\sin \left(k r_{i j}\right)}{k r_{i j}}\left\langle\delta \boldsymbol{\psi}_{i}\left(t_{0}\right) \cdot \delta \boldsymbol{\psi}_{j}\left(t_{0}+t\right)\right\rangle_{t_{0}}, \\
\langle\cdots\rangle_{t_{0}} & =\frac{1}{T_{\max }-t} \sum_{t_{0}=1}^{T_{\max }-t}(\cdots),
\end{aligned}
$$

with $T_{\max }$ being the length of the simulation. The number of operations needed to calculate this quantity is in general $\sim T_{\max } N^{2}$; however, what we actually need for the scaling analysis is the correlation function at $k=0$ (see Sec. V B 2), which is numerically less demanding:

$$
\begin{aligned}
C(k=0, t) & =\frac{1}{T_{\max }-t} \sum_{t_{0}=1}^{T_{\max }-t} \overline{\delta \psi\left(t_{0}\right)} \cdot \overline{\delta \psi\left(t_{0}+t\right)}, \\
\overline{\delta \psi\left(t_{0}\right)} & =\frac{1}{N} \sum_{i} \delta \boldsymbol{\psi}_{i}\left(t_{0}\right) .
\end{aligned}
$$

From this quantity, we computed the characteristic timescale $\tau$ from the definition

$$
\frac{1}{2 \pi}=\int_{0}^{\infty} d t \frac{1}{\tau} \sin \left(\frac{t}{\tau}\right) \frac{C(k=0, t)}{C(k=0, t=0)} .
$$

This condition corresponds to requiring that half of the total integrated area of the dynamic correlation function in the frequency domain comes from the interval $-\omega_{c}<\omega<\omega_{c}$, with $\omega_{c}=1 / \tau$. This definition of $\tau$ has the advantage of capturing the relevant timescale both when relaxation is dissipative and when propagating modes are present, and it is the standard definition adopted in the literature on dynamic critical phenomena [16].

\section{Dynamic crossover}

Our primary objective is to observe the crossover in the dynamic critical behavior predicted by the RG computation. According to Eq. (81), there are two dynamical regimes at small and large values of $\xi$, separated by a crossover length scale fixed by $\mathcal{R}_{0}$. The simplest protocol to check this scenario in numerical simulations would seem to fix the value of the dissipation coefficient (and therefore $\mathcal{R}_{0}$ ) and extensively vary the correlation length by tuning $T$. Since $\tau \sim \xi^{z}$, when plotting $\tau$ versus $\xi$, we should then observe two different power laws, one with exponent $z=d / 2$ for small $\xi$ and another one with $z=2$ at large $\xi$. The problem with this protocol is that to see a change in the power-law exponent one must span several orders of magnitudes in $\xi$, three decades at the very least. However, $L=10^{3}$ gives $N=10^{9}$ in $d=3$, which is quite demanding numerically, considering that the largest relaxation time would be of order $\tau \sim \xi^{2} \sim L^{2} \sim 10^{6}$. This is not possible, and the maximum size we used is well below $(L \leqslant 20)$.

In other terms, simulations at a single value of $\hat{\eta}$ are unlikely to show clearly the crossover.

To better illustrate this point, in Fig. 4 we have reproduced the $\left(\xi, \mathcal{R}_{0}\right)$ plane of Fig. 2, which describes the crossover 


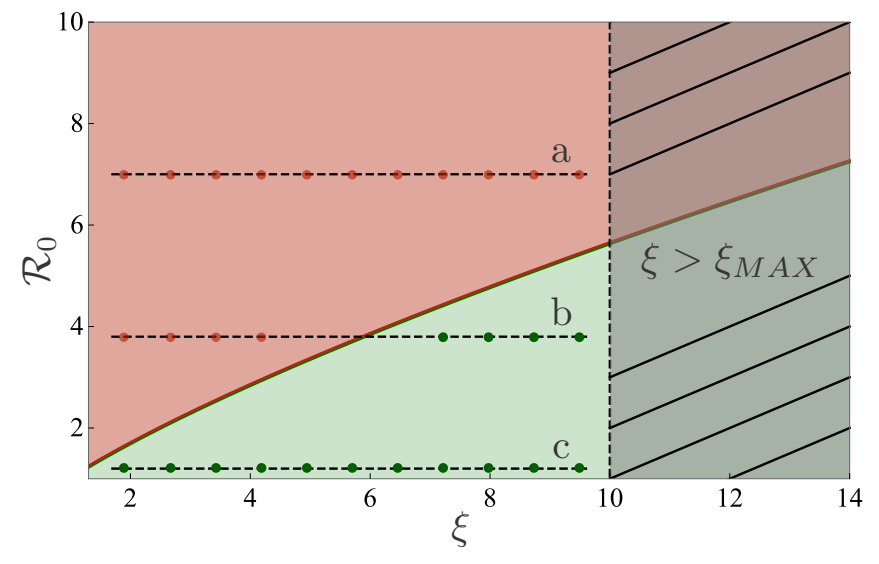

FIG. 4. Numerical protocol in the $\left(\xi, \mathcal{R}_{0}\right)$ plane. Depending on the values of $\xi$ and $\mathcal{R}_{0}$, the system explores two different critical regimes (see also Fig. 2). Simulations performed at fixed $\hat{\eta}$ and different $T$ correspond to exploring the $\left(\xi, \mathcal{R}_{0}\right)$ plane along horizontal segments. Since the size of the system is finite $(L \leqslant 20)$, only a limited window of $\xi$ can be accessed and the length of such segments is finite $\left(1=\Lambda^{-1}<\xi<\xi_{\max }=10\right)$. According to the RG prediction, for all values of $\hat{\eta}$ corresponding to $\mathcal{R}_{0}>10^{3 / 4}$ the segments belong entirely to the conservative region (segment a). For larger values of $\hat{\eta}$, such that $1<\mathcal{R}_{0}<10^{3 / 4}$, the segments cross from the conservative region to the dissipative one (segment $b$ ): In this case, there is no sufficient span in each region to extract the exponent $z$ from the $\tau$ vs $\xi$ plot. Since the minimum physical value of $\mathcal{R}_{0}$ is $\Lambda^{-1}=1$, larger values of $\hat{\eta}$ are all equivalent to the $\mathcal{R}_{0}=1$ case (segment c).

predicted by the RG computation, and we have explicitly marked the physical constraints related to the simulated microscopic model. Since the maximum simulated size is $L=$ 20 , the region with $\xi>\xi_{\max } \approx L / 2=10$ is unaccessible. Besides, the lattice spacing we use is $\Lambda^{-1}=1$, and both $\xi$ and $\mathcal{R}_{0}$ must then be considered starting from such physical value. Numerical simulations at fixed $\hat{\eta}$ (i.e., fixed $\mathcal{R}_{0}$ ) therefore correspond in this diagram to finite horizontal intervals that we depicted with dashed dotted lines in Fig. 4. The value of $\hat{\eta}$ used in simulations determines the value of $\mathcal{R}_{0}$ (larger values of the first one mean lower values of the second), i.e., the value at which the horizontal lines run in the figure. Upon increasing $\hat{\eta}, \mathcal{R}_{0}$ will at some point reach its minimal value $\Lambda^{-1}=1$ and all larger $\hat{\eta}$ therefore correspond to the same boundary $\mathcal{R}_{0}=1$ interval [Fig. 4(c)].

Figure 4 clearly shows that there is only a narrow range of $\mathcal{R}_{0}$ where the horizontal interval crosses the crossover line [Fig. 4(c)], and here the span within each regime is by far too small to appropriately extract $z$ from a power law fit. On the contrary, in most cases the horizontal interval entirely lies within the same dynamic regime [Figs. 4(a) and 4(c)]. In this case, in numerical simulations at fixed $\hat{\eta}$ we should expect that only one power law is observed in the $\tau$ versus $\xi$ plot. For this reason, the best way to capture the presence of the crossover is to run simulations at several different values of the effective friction. If the RG picture is correct, when $\hat{\eta}$ is small, corresponding to large $\mathcal{R}_{0}$, we should measure $z=3 / 2$ [Fig. 4(a)], while for large enough $\hat{\eta}$ we should measure $z=2$ [Fig. 4(c)].

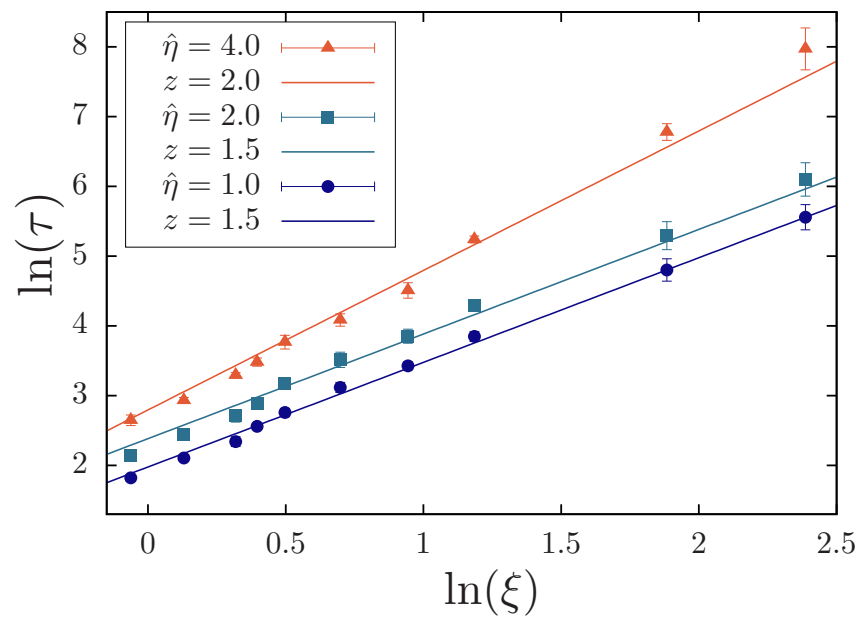

FIG. 5. Dynamic critical exponents. Relaxation time versus correlation length in $d=3$, for $L=20, N=8000$, and $T \in[1.48$ : 2.00], at various values of the friction coefficient, $\hat{\eta}=1.0,2.0,4.0$. Each point is an average over 10 samples, apart from the lowest $T$ (largest $\xi$ and $\tau$ ) at $\hat{\eta}=4$, for which we have four samples (one such sample takes 7 days to run on a $7-8700,3.20 \mathrm{GHz}$ CPU). Lines are the best fit to $z=1.5$ (low friction, $\hat{\eta}=1.0,2.0$ ) and $z=2$ (large friction, $\hat{\eta}=4.0$ ).

The numerical findings fully confirm this scenario. In Fig. 5, we show results for three different sets of simulations, respectively, for $\hat{\eta}=1,2$, 4. We cannot use larger values for the effective dissipation because the maximum relaxation time becomes too long to equilibrate the system. For the smaller values $(\hat{\eta}=1,2)$, the data are in good agreement with a dynamic critical exponent $z=3 / 2$, while for $\hat{\eta}=4$ the characteristic timescales with the correlation length with an exponent $z=2$. We therefore conclude that the ISM exhibits a dynamic crossover in critical behavior, as predicted by the RG.

To further support the existence of two distinct regimes in dynamical behavior, we tested the full dynamic scaling hypothesis (8) on the dynamic correlation functions. In Fig. 6, upper panels, we display the normalized $C(k=0, t)$ for all the temperatures that we analyzed, and for two different values of $\hat{\eta}$. In the lower panels, we report the same curves but plotted as a function of the rescaled variable $t / \xi^{z}$, where we used the values of $z$ obtained from the previous analysis. The figure shows that dynamic scaling is nicely verified, but with different exponents ( $z=3 / 2$ and $z=2$, respectively) at small and large values of the friction coefficient.

\section{Natural swarms and inertial dynamics}

Both theoretical computations and numerical simulations describe a dynamic crossover ruled by the interplay of the correlation length $\xi$ and the conservation length scale $\mathcal{R}_{0}$. This analysis has important consequences regarding systems of finite size. What we have shown is that, even in the presence of dissipation, the critical behavior can be ruled by conservative critical dynamics with exponent $z=3 / 2$ (in $d=3$ ) in a wide region of parameters (case $\mathcal{R}_{0}>\xi^{3 / 4}$; red region in Fig. 4). This result is particularly relevant if we think back at the biological motivation of our study: explaining experimental 

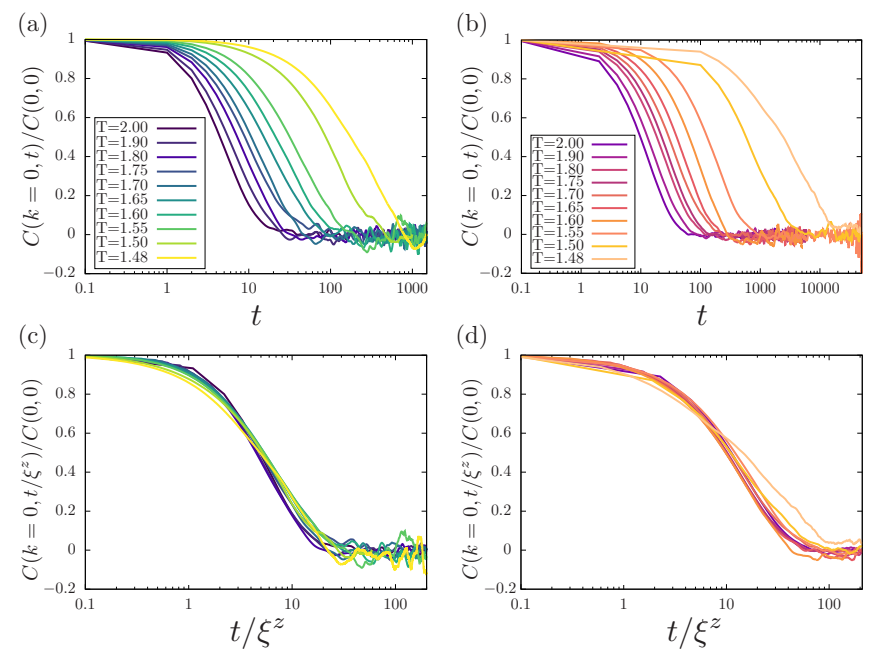

FIG. 6. Dynamic scaling for correlations. Test of the dynamic scaling hypothesis on the dynamic correlation functions at $k=0$. Upper panels: spatiotemporal correlation functions at various values of the temperature for $\hat{\eta}=1$ (a) and $\hat{\eta}=4$ (b). Lower panels: same curves plotted as a function of $t / \xi^{z}$ with, respectively, $z=1.5$ (c) and $z=2$ (d): In both cases, the functions verify the dynamic scaling hypothesis.

data in natural swarms of insects. As discussed in Sec. II, swarms exhibit dynamic scaling, but with an exponent smaller than the one predicted by models of collective motion with a purely dissipative dynamics. This is why we considered the ISM in the first place: to put back inertial terms in the dynamical equations and to understand whether they can produce $z<2$ on the collective scale of living groups. The answer to this question is therefore yes. The exponent that we get in the conservative region, $z=1.5$, is not yet that observed in the data $(z \approx 1.2)$, but it is a big step forward as compared to the prediction of the Vicsek model $(z=2)$. This strongly indicates that the ISM captures an important ingredient-inertia-absent in previous models.

Numerical simulations of the ISM also reproduce another feature measured in natural swarms, which is not reproduced by previous models. Experimental correlation functions in natural swarms display a concave shape at short times, incompatible with the exponential relaxation of the Vicsek model [22]. The ISM, on the other hand, displays the same kind of behavior as in the swarms data. To show this, in Fig. 7(a) we compare the dynamical relaxation of natural swarms with simulations of the ISM and of the Vicsek model in the paramagnetic phase. We can see that ISM reproduces the curvature of the experimental correlation for $t \rightarrow 0$, contrary to the Vicsek model. The consistency between ISM and natural swarms becomes even more striking when we compute the relaxation form factor [22],

$$
h(t / \tau) \equiv \frac{\dot{C}(t / \tau)}{C(t / \tau)} .
$$

The limit of this function for $t \rightarrow 0$ is equal to 1 if the dynamics is purely exponential, as in the case of the Vicsek model. On the other hand, an inertial dynamics approaches zero for small times: This is the case of ISM and of natural swarms.
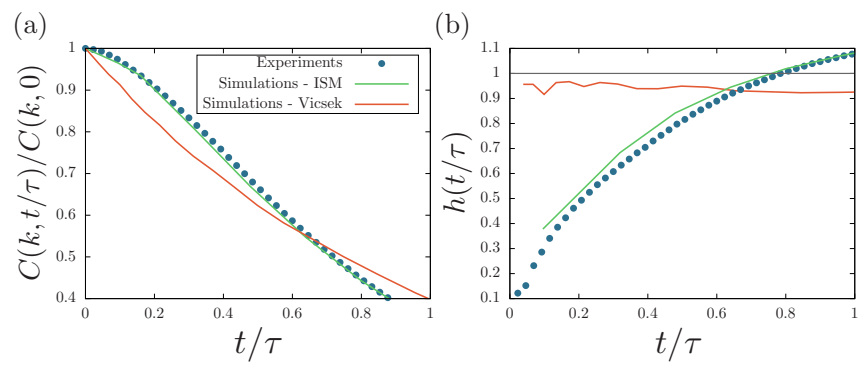

FIG. 7. Inertial behavior: experiments vs models. (a) Normalized dynamical correlation functions $C(k, t) / C(k, 0)$ at nonzero values of $k$; in all three cases, $k$ has been chosen in such a way to have $k \xi=1$, to reproduce the scaling situation found in experiments on natural swarms [22] (Vicsek swarm $k=0.717$, natural swarm $k=$ 0.798 , and ISM $k=0.673$ ). (b) The relaxation form factor $h(t / \tau) \equiv$ $\dot{C}(t / \tau) / C(t / \tau)$ goes to 1 for overdamped exponential relaxation, while it goes to 0 for inertial relaxation [22]. The fixed-network ISM reproduces the correlation form of real swarms in a rather compelling way.

We therefore conclude that the ISM in the paramagnetic phase qualitatively well describes the inertial dynamics of natural swarms.

\section{CONCLUSIONS}

We have performed a one-loop RG calculation of the critical dynamics of a statistical system with inertial nondissipative couplings in the presence of a dissipative term which violates the conservation law of the symmetry generator. Our calculation was motivated by recent experiments on the collective dynamics of natural swarms of insects [22], although the dynamical field equations we studied are relevant also for BEC systems with terms weakly violating the symmetry in the Hamiltonian [29-31]. We find that the RG flow has two fixed points: a conservative yet unstable one and a dissipative stable one, associated to the dynamical critical exponents $z=d / 2$ and $z=2$, respectively. The crossover between the two fixed points is regulated by a conservation length scale, $\mathcal{R}_{0}$ : For scales much larger than $\mathcal{R}_{0}$, the dynamics is ruled by the dissipative fixed point, while for scales smaller than $\mathcal{R}_{0}$ critical slowdown is governed by the conservative fixed point. Numerical simulations on the microscopic model confirm our results.

The length scale $\mathcal{R}_{0}$ is given by the ratio between the transport coefficient, $\lambda_{0}$, and the effective friction, $\eta_{0}$, of the spin field. While the coarse-grained parameter $\eta_{0}$ is certainly connected to its microscopic counterpart, $\hat{\eta}$, of the original model, the same cannot be said for the transport coefficient: In the microscopic model, there is no transport term. The interesting fact, then, is that the conservative transport term, $\lambda_{0} \nabla^{2} s$, is generated by the renormalization group through the spin self-energy $\Pi$ at one loop. Therefore, we are in one of those rare cases in which a crucial length scale of the system, i.e., $\mathcal{R}_{0}$, cannot be guessed purely on the basis of dimensional analysis of the microscopic equations of motion (possibly with some renormalized anomalous dimensions). Of course, one could have guessed (admittedly rather smartly) that the presence of a symmetry and conservation law, albeit violated 
by $\eta_{0}$, should require a conservative transport term. But even if our intuition is not so good, the RG requires by itself the existence of such term and therefore the emergence of a crossover length scale, thus confirming its power in dictating what is relevant and what is not in strongly correlated systems.

The fact that the crossover length scale $\mathcal{R}_{0}$ is larger as the dissipation is smaller has important consequences for biological systems. Real biological groups always have finite size; hence, in order to study their behavior we cannot just take the hydrodynamic limit (infinitely large times and lengths) for granted but we have to cope with the actual size of the system. In both flocks and swarms, experiments have shown that dissipative terms are rather weak [22,27], suggesting that the conservation length scale $\mathcal{R}_{0}$ is quite large. Under these circumstances, one may have a conservation length scale that is larger than the system's size, $\mathcal{R}_{0}>L$. In this case, one would find a dynamical critical exponent equal to that of the fully conservative RG fixed point, namely $z=3 / 2$ in $d=$ 3 , and a dynamic correlation function with strong signature of nonexponential inertial relaxation. Thanks to this finitesize critical crossover, the fully conservative phenomenology should hold at all practically attainable values of the correlation length, which is always limited by the system's size.

From the point of view of the comparison between theory and experiments in natural swarms, our calculation therefore puts us in a semisatisfactory situation. Certainly, we can say that the form of the dynamical correlation functions of natural swarms and in particular the nonexponential inertial nature of the short time dynamics, is rendered by the ISM in a much more compelling way than by the Vicsek model (Fig. 7), and actually our simulations show almost no quantitative difference between theory and experiments in this respect. Concerning the dynamical critical exponent, $z$, the situation is still open, although we would say that the result of the present calculation $-z=3 / 2$ for finite-size weakly damped swarms in $3-d$-definitely goes in the right direction. Experiments give $z \approx 1.2$, even though values up to $z=1.3$ would probably be acceptable, given the noise in the data [22]. On the other hand, the Vicsek model, and in fact any model dominated at short times by purely dissipative dynamics, gives $z \approx 2$. This is quite understandable, as all these models belong (at equilibrium) to the same dynamical universality class as classic Heisenberg (model A of Ref. [23]), which has $z=2$ at the one-loop level, with very small corrections at two loops [23]. Moreover, when off-equilibrium (self-propelled) effects are taken into account, numerical simulations performed over time and space scales comparable to real swarms still give $z \approx$ 2 [22], completely incompatible with the data. The present calculation, on the other hand, shows that once nondissipative terms are introduced in the dynamics, provided that dissipation is not too strong, the dynamical critical exponent changes to $z=d / 2$. This is a value significantly closer to the experimental exponent than that of purely dissipative models. Hence, it seems to us that nondissipative terms are important to reproduce the correct critical dynamics of real swarms.

Of course, one must now ask how to bridge the gap between the present $\mathrm{RG}$ exponent and the experimental value $z \approx 1.2$. There are several possibilities. First, one should try to have more statistics in the experiments, possibly with larger swarms, to check whether the data are really inconsistent with $z=3 / 2$; work in this direction requires considerable technical effort on the experimental side (in particular, higher definition and faster acquisition systems). Second, one may hope that a two-loop calculation improves things. We are not very optimistic in this respect, though. Normally, two-loop corrections to the exponents are quite small, so it seems hard to bridge the gap between 1.5 and 1.2 in this way; furthermore, in the nondissipative case the value $z=3 / 2$ is actually valid at all order of the perturbative series, courtesy of the Ward identities generated by the symmetry [43]. Although in our case there is dissipation, we suspect that, as long as the system is in the proximity of the conservative $\mathrm{RG}$ fixed point, $z=3 / 2$ will resist any attempt to be perturbatively changed.

Finally, there is the third and most promising source of corrections to $z$, namely off-equilibrium effects due to the self-propulsion of the individuals. Even though these are not sufficient to change the critical exponent in the Vicsek model, it could be that the compound effect of having nondissipative inertial couplings and a self-propelled dynamics, further shifts the exponent in the correct direction. Studying this case from the theoretical point of view (i.e., with RG) will be quite nontrivial, as one needs to use the approach of Toner and Tu [19], including in the theory one extra field, the density, coupled to velocity and spin, much as it has been done in Ref. [39] for the low-temperature phase. However, at low temperature one could exploit the spin-wave expansion to linearize the equations, while close to $T_{c}$ one needs to fully take into account the nonlinearities through the RG. Performing even a one-loop calculation with three fields (which become six once the Martin-Siggia-Rose functional is introduced) really does not look like a piece of cake. Still, one should try. In the meanwhile, numerical simulations of the full-fledged self-propelled ISM close to criticality should be performed, to see from the data if there is case for hope.

Actually, we have some reasons to be optimistic. The fact that models with nondissipative terms have dynamical critical exponent $z$ significantly smaller than the purely dissipative value 2 may be interpreted as a critical counterpart of the linear spin-wave behavior at low $T$ : In this regime, "second sound" modes propagate linearly, with dispersion relation $\omega=c k$ [38]. Naively, this relation would suggest $z=1$ for these systems, but this is not the case because close to $T_{c}$ parameters renormalize and the second sound speed, $c$, goes to zero as some function of $k$ : This RG-induced $k$ dependence changes the exponent from the trivial 1 , to the final $z=3 / 2$ in this kind of models [23]. Despite this correction, though, the exponent remains significantly lower than the purely dissipative 2, as a relic of the low-temperature spin-wave dynamics. We may hope that a similar mechanism will be at work when self-propulsion will be taken into account. The first obvious effect of self-propulsion on a system with nondissipative mode-coupling terms is to produce ballistic (i.e., linear) motion of each individual, even in the disordered collective phase. The dynamic critical exponent does not measure the motion of the individuals, of course, but rather the relaxation law of the velocity fluctuations; however, similar to what happens with the renormalization of linear spin waves, one may hope that some relic of the ballistic regime creeps into the critical phase calculation of $z$, thus lowering it below the static equilibrium value $3 / 2$ and eventually bringing it closer 
to the experimental value. Further experimental, numerical, and theoretical effort will tell whether this educated guess is just wishful thinking.

\section{ACKNOWLEDGMENTS}

We warmly thank Enzo Branchini, Antonio Culla, Erwin Frey, and Luca Peliti for important discussions; Lara Benfatto, Claudio Castellani, and Jose G. Lorenzana for suggestions about BEC systems; and Stefania Melillo for help with the experimental data of Ref. [22]. This work was supported by ERC Advanced Grant RG.BIO (Contract No. 785932) to A.C. and ERANET-CRIB Grant to A.C. and T.S.G. T.S.G. was also supported by grants from CONICET, ANPCyT, and UNLP (Argentina).

\section{APPENDIX A: PERTURBATION EXPANSION}

\section{Martin-Siggia-Rose formalism}

The Martin-Siggia-Rose (MSR) formalism is a method to write stochastic differential equations as a field theory formulated using path integrals. The core idea is that when computing thermal averages of all possible field configurations, only those satisfying the original equations do contribute. One can select such configurations using a Dirac $\delta$ functional: Suppose that we want to select only configurations of the field $\boldsymbol{\psi}$ that satisfy the equation $\mathcal{F}(\boldsymbol{\psi})-\boldsymbol{\theta}=0$, where $\mathcal{F}(\boldsymbol{\psi})$ generically describes the deterministic part of the equation (i.e., time derivatives, differential operators, interaction terms, coupling with other fields, etc.) and $\boldsymbol{\theta}$ is the stochastic noise. If $\operatorname{det}(\delta \mathcal{F} / \delta \boldsymbol{\psi})=1$, which is the case for stochastic
Langevin equations in the Ito representation [24,61], we can write

$$
1=\int \mathcal{D} \psi(\mathbf{x}, t) \delta(\mathcal{F}(\psi(\mathbf{x}, t))-\boldsymbol{\theta}(\mathbf{x}, t)) .
$$

We can introduce the field $\hat{\psi}(x, t)$ and use the integral representation of the Dirac $\delta$ functional:

$$
1=\int \mathcal{D} \boldsymbol{\psi} \mathcal{D} \hat{\psi} e^{\left\{-i \int d^{d} x \int d t \hat{\psi}(\mathbf{x}, t)[\mathcal{F}(\boldsymbol{\psi}(\mathbf{x}, t))-\boldsymbol{\theta}(\mathbf{x}, t)]\right\}} .
$$

The field $\hat{\psi}$ may also be interpreted as a Lagrange multiplier, since it is introduced to select given values, or rather configurations, of the field $\psi$. In our case, since (15) and (16) are two coupled equations for two distinct fields, we need to implement two $\delta$ functions, and therefore introduce two auxiliary fields $\hat{\boldsymbol{\psi}}$ and $\hat{\boldsymbol{s}}$. The above identity can then be averaged over the distribution of the stochastic noises, leaving with an effective functional measure that can be used to compute thermal averages, i.e.,

$$
\begin{gathered}
1=\int \mathcal{D} \boldsymbol{\psi} \mathcal{D} \hat{\boldsymbol{\psi}} \mathcal{D} \boldsymbol{s} \mathcal{D} \hat{\boldsymbol{s}} e^{-\mathcal{S}[\boldsymbol{\psi}, \hat{\boldsymbol{\psi}}, \boldsymbol{s}, \hat{\boldsymbol{s}}]}, \\
\langle f\rangle=\int \mathcal{D} \boldsymbol{\psi} \mathcal{D} \hat{\boldsymbol{\psi}} \mathcal{D} \boldsymbol{s} \mathcal{D} \hat{\boldsymbol{s}} f e^{-\mathcal{S}[\boldsymbol{\psi}, \hat{\boldsymbol{\psi}}, \boldsymbol{s}, \hat{\boldsymbol{s}}]},
\end{gathered}
$$

where $f$ is a generic function of the fields. After standard manipulations [24], we get

$\mathcal{S}[\boldsymbol{\psi}, \hat{\boldsymbol{\psi}}, \boldsymbol{s}, \hat{\boldsymbol{s}}]=\mathcal{S}_{0, \psi}[\hat{\boldsymbol{\psi}}, \boldsymbol{\psi}]+S_{0, s}[\hat{\boldsymbol{s}}, \boldsymbol{s}]+\mathcal{S}_{I}[\boldsymbol{\psi}, \hat{\boldsymbol{\psi}}, \boldsymbol{s}, \hat{\boldsymbol{s}}]$.

Here, $\mathcal{S}_{0, \psi}$ and $\mathcal{S}_{s, 0}$ are Gaussian free actions respectively for the fields $\psi$ and $s$ and are given by

$$
\begin{aligned}
& \mathcal{S}_{0, \psi}=\int \frac{d^{d} k}{(2 \pi)^{d}} \frac{d \omega}{2 \pi} \hat{\psi}_{\alpha}(-\mathbf{k},-\omega)\left[-i \omega+\Gamma_{0}\left(k^{2}+r_{0}^{2}\right)\right] \psi_{\alpha}(\mathbf{k}, \omega)+\Gamma_{0} \hat{\psi}_{\alpha}(-\mathbf{k},-\omega) \hat{\psi}_{\alpha}(\mathbf{k}, \omega), \\
& \mathcal{S}_{0, s}=\int \frac{d^{d} k}{(2 \pi)^{d}} \frac{d \omega}{2 \pi} \hat{s}_{\alpha}(-\mathbf{k},-\omega)\left[-i \omega+\left(\eta_{0}+\lambda_{0} k^{2}\right)\right] s_{\alpha}(\mathbf{k}, \omega)+\left(\eta_{0}+\lambda k^{2}\right) \hat{s}_{\alpha}(-\mathbf{k}, \omega) \hat{s}_{\alpha}(\mathbf{k}, \omega),
\end{aligned}
$$

where Greek letters stand for space coordinates and repeated indices are summed. The interaction term involves both $\boldsymbol{s}, \hat{\boldsymbol{s}}$ and $\boldsymbol{\psi}$, $\hat{\psi}$ and is given by

$$
\begin{aligned}
\mathcal{S}_{I}= & -g_{0} \epsilon_{\alpha \beta \gamma} \int \frac{d^{d} k_{1}}{(2 \pi)^{d}} \frac{d^{d} k_{2}}{(2 \pi)^{d}} \frac{d \omega_{1}}{2 \pi} \frac{d \omega_{2}}{2 \pi}\left(k_{2}^{2}-k_{1}^{2}\right) \psi_{\gamma}\left(\mathbf{k}_{2}, \omega_{2}\right) \psi_{\beta}\left(\mathbf{k}_{1}, \omega_{1}\right) \hat{s}_{\alpha}\left(-\mathbf{k}_{1}-\mathbf{k}_{2},-\omega_{1}-\omega_{2}\right) \\
& -\frac{g_{0}}{2} \epsilon_{\alpha \beta \gamma} \int \frac{d^{d} k_{1}}{(2 \pi)^{d}} \frac{d^{d} k_{2}}{(2 \pi)^{d}} \frac{d \omega_{1}}{2 \pi} \frac{d \omega_{2}}{2 \pi} \hat{\psi}_{\alpha}\left(\mathbf{k}_{1}, \omega_{1}\right) \psi_{\beta}\left(\mathbf{k}_{2}, \omega_{2}\right) s_{\gamma}\left(-\mathbf{k}_{1}-\mathbf{k}_{2},-\omega_{1}-\omega_{2}\right) \\
& -2 \Gamma_{0} u_{0} \int \frac{d^{d} k_{1}}{(2 \pi)^{d}} \frac{d^{d} k_{2}}{(2 \pi)^{d}} \frac{d^{d} k_{3}}{(2 \pi)^{d}} \frac{d \omega_{1}}{2 \pi} \frac{d \omega_{2}}{2 \pi} \frac{d \omega_{3}}{2 \pi} \hat{\psi}_{\alpha}\left(\mathbf{k}_{1}, \omega_{1}\right) \psi_{\alpha}\left(\mathbf{k}_{2}, \omega_{2}\right) \psi_{\alpha}\left(\mathbf{k}_{3}, \omega_{3}\right) \psi_{\alpha}\left(-\mathbf{k}_{1}-\mathbf{k}_{2}-\mathbf{k}_{3},-\omega_{1}-\omega_{2}-\omega_{3}\right)
\end{aligned}
$$

From the free part of the action, Eqs. (A6) and (A7), we immediately read the expressions for the bare propagators and correlation functions for the effective field theory, which coincide with Eqs. (23), (24) and (27), (28), respectively:

$$
\begin{gathered}
\left\langle\psi_{\alpha}(-\mathbf{k},-\omega) \hat{\psi}_{\beta}(\mathbf{k}, \omega)\right\rangle=\delta_{\alpha \beta} G_{0, \psi}(\mathbf{k}, \omega)=\delta_{\alpha \beta}\left[-i \omega+\Gamma_{0}\left(k^{2}+r_{0}\right)\right]^{-1}, \\
\left\langle\psi_{\alpha}(-\mathbf{k},-\omega) \psi_{\beta}(\mathbf{k}, \omega)\right\rangle=\delta_{\alpha \beta} C_{0, \psi}(\mathbf{k}, \omega)=2 \delta_{\alpha \beta} \Gamma_{0}\left|G_{0, \psi}\right|^{2}, \\
\left\langle s_{\alpha}(-\mathbf{k},-\omega) \hat{s}_{\beta}(\mathbf{k}, \omega)\right\rangle=\delta_{\alpha \beta} G_{0, s}(\mathbf{k}, \omega)=\delta_{\alpha \beta}\left[-i \omega+\left(\eta_{0}+\lambda_{0} k^{2}\right)\right]^{-1}, \\
\left\langle s_{\alpha}(-\mathbf{k},-\omega) s_{\beta}(\mathbf{k}, \omega)\right\rangle=\delta_{\alpha \beta} C_{0, s}(\mathbf{k}, \omega)=2 \delta_{\alpha \beta}\left(\eta_{0}+\lambda_{0} k^{2}\right)\left|G_{0, s}\right|^{2} .
\end{gathered}
$$


These functions are the building blocks of the perturbative expansion: Full correlation functions and propagators can be written in terms of these bare averages. At this point, standard Feynman rules can be applied to carry out the perturbation theory.

From the form of the interacting part of the action, we can see that there are two kinds of dynamic vertices, namely

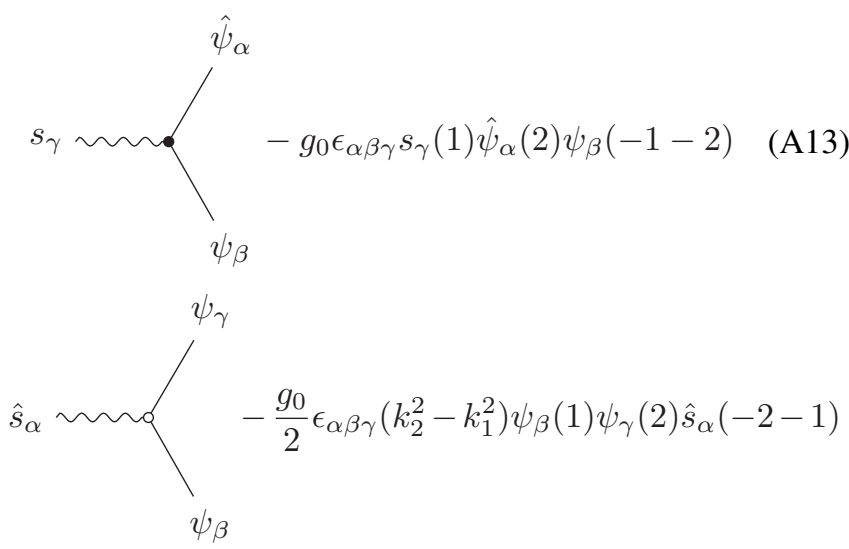

(A14)

Here we are representing with a solid line the fields $\boldsymbol{\psi}, \hat{\psi}$, and with wavy lines the fields $\boldsymbol{s}, \hat{\boldsymbol{s}}$; for clarity, we are indicating with $\pm n$ the dependence of the fields on wave number and frequency: $\psi_{\alpha}( \pm n)=\psi_{\alpha}\left( \pm \mathbf{k}_{n}, \pm \omega_{n}\right)$. Besides these two dynamic vertices, there is also a static vertex involving the coupling $u_{0}$. Since we are focusing on the contributions of purely dynamic origin to the perturbative expansion, we do not concern ourselves with that vertex in our discussion. We just take it for granted that the perturbation expansion related to $u_{0}$ gives back the terms of the equilibrium theory and refer the reader to the standard literature for more details [24,47].

At this level, we should notice that the second vertex carries with it an important factor $\left(k_{2}^{2}-k_{1}^{2}\right)$. This is a consequence of the reversible couplings between the field $\psi$ and $s$ present in the equations of motion. Its origin lies in the symmetries of the system: The spin is the generator of rotations of the order parameter and, consequently, the reversible couplings between the fields occur just via a cross product. The field $\hat{s}$ therefore couples only with $(\nabla \boldsymbol{\psi})^{2}$; for this reason the vertex is proportional to $\left(k_{2}^{2}-k_{1}^{2}\right)$, and this implies that every diagram with an $\hat{\boldsymbol{s}}(\mathbf{k}=0, \omega)$ external line vanishes.

\section{Perturbation expansion at one loop}

To compute average quantities with the measure (A4), one proceeds as usual to develop the exponential contribution due to the interaction action, being left with a perturbation expansion where only free propagators and free correlations appear connected to each other through the various interaction vertices. When building the full averages in such a way, we have to take into account that both $\langle\hat{\boldsymbol{\psi}} \boldsymbol{\psi}\rangle_{0}$ and $\langle\boldsymbol{\psi} \boldsymbol{\psi}\rangle_{0}$ are nonzero. To graphically distinguish between them, we will represent the propagators with an arrow and the correlation functions with a line, since propagators are time ordered while correlation functions are not. We will use the same rules also for propagators and correlation functions of $s$, but using wavy lines. It is more convenient to write down the perturbative expansion of $G$ using the Dyson equation [62]:

$$
\begin{aligned}
G_{\psi}^{-1}(\mathbf{k}, \omega)_{\alpha \beta} & =G_{0, \psi}^{-1}(\mathbf{k}, \omega) \delta_{\alpha \beta}-\Sigma_{\alpha \beta}(\mathbf{k}, \omega), \\
G_{s}^{-1}(\mathbf{k}, \omega)_{\alpha \beta} & =G_{0, s}^{-1}(\mathbf{k}, \omega) \delta_{\alpha \beta}-\Pi_{\alpha \beta}(\mathbf{k}, \omega),
\end{aligned}
$$

for which we use the following diagrammatic notation:

$$
\begin{gathered}
G_{0, \psi_{\alpha, \beta}}=\longrightarrow, C_{0, \psi_{\alpha, \beta}}=\longrightarrow, \\
G_{0, s_{\alpha, \beta}}=\leadsto, C_{0, \psi_{\alpha, \beta}}=\sim \hat{\psi}_{\beta}, \\
\Sigma_{\alpha, \beta}=\psi_{\alpha} \leadsto \hat{s}_{\beta} . \\
\Pi_{\alpha, \beta}=s_{\alpha}
\end{gathered}
$$

Here, the blob indicates the sum of all one particle irreducible (1PI) diagrams with an incoming $\boldsymbol{\psi}$ (or $s$ ) field and an outgoing $\hat{\boldsymbol{\psi}}$ (or $\hat{\boldsymbol{s}}$ ) field and with amputated external legs: namely, the self-energies $\Sigma_{\alpha \beta}$ and $\Pi_{\alpha \beta}$.

The diagrammatic expressions for the self-energies of $s$ and $\psi$ at one loop are

$$
\begin{aligned}
& \Sigma_{\alpha \beta}=\psi_{\alpha} \rightarrow \hat{\psi}_{\beta}+\psi_{\alpha} \hat{q}-\hat{\psi}_{\beta}, \\
& \Pi_{\alpha \beta}=s_{\alpha} \leadsto \rho \hat{s}_{\beta} \text {, }
\end{aligned}
$$

where external legs are amputated. It is possible to translate these diagrams into integrals using standard Feynman diagrams rules:

$$
\begin{gathered}
\Sigma_{\alpha \beta}(\mathbf{k}, \omega)=-2 g_{0}^{2} \delta_{\alpha \beta} \int \frac{d^{d} p}{(2 \pi)^{d}} \int \frac{d \omega^{\prime}}{2 \pi}\left[G_{0, \psi}\left(\mathbf{p}, \omega^{\prime}\right) C_{0, s}\left(\mathbf{k}-\mathbf{p}, \omega-\omega^{\prime}\right)+\left(k^{2}-p^{2}\right) C_{0, \psi}\left(\mathbf{p}, \omega^{\prime}\right) G_{0, s}\left(\mathbf{k}-\mathbf{p}, \omega-\omega^{\prime}\right)\right], \\
\Pi_{\alpha \beta}(\mathbf{k}, \omega)=-2 g_{0}^{2} \delta_{\alpha \beta} \int \frac{d^{d} p}{(2 \pi)^{d}} \int \frac{d \omega^{\prime}}{2 \pi}\left[C_{0, \psi}\left(\mathbf{p}, \omega^{\prime}\right) G_{0, \psi}\left(\mathbf{k}-\mathbf{p}, \omega-\omega^{\prime}\right)\left((\mathbf{k}-\mathbf{p})^{2}-p^{2}\right)\right] .
\end{gathered}
$$

Performing the frequency integration, we get the following expressions for the self-energies:

$$
\begin{gathered}
\Sigma_{\alpha \beta}(\mathbf{k}, \omega)=-2 g_{0}^{2} \delta_{\alpha \beta} \int \frac{d^{d} p}{(2 \pi)^{d}} \frac{\left(k^{2}+r_{0}\right)}{\left(p^{2}+r_{0}\right)\left(-i \omega+\Gamma_{0}\left(p^{2}+r_{0}\right)+\lambda_{0}(\mathbf{k}-\mathbf{p})^{2}+\eta_{0}\right)}, \\
\Pi_{\alpha \beta}(\mathbf{k}, \omega)=-g_{0}^{2} \delta_{\alpha \beta} \int \frac{d^{d} p}{(2 \pi)^{d}} \frac{\left[p^{2}-(\mathbf{k}-\mathbf{p})^{2}\right]^{2}}{\left(p^{2}+r_{0}\right)\left((\mathbf{k}-\mathbf{p})^{2}+r_{0}\right)} \frac{1}{\left(-i \omega+\Gamma_{0}\left(p^{2}+(\mathbf{k}-\mathbf{p})^{2}+2 r_{0}\right)\right)},
\end{gathered}
$$


We thus find that the self-energies only have, as expected, a diagonal non-zero contribution for $\alpha=\beta$. We shall therefore drop the coordinate index and simply indicate them as $\Sigma$ and $\Pi$, as in Eqs. (31) and (32) in the main text. When the perturbative corrections are calculated integrating over the shell, as in the RG approach we use in Sec. IV, the integrals are performed between $\Lambda / b$ and $\Lambda$. On the other hand, in the Callan-Symanzik approach of Sec. VI, all $p$ integrals are performed between 0 and $\infty$.

\section{APPENDIX B: VERTEX CORRECTIONS}

It can be shown that the dynamic coupling constant $g_{0}$ has no perturbative contributions at all orders of perturbation theory. At one loop, the correction $\Delta g_{\alpha \beta \gamma}^{(1)}$, of order $g_{0}^{3}$, to vertex (A13) comes from these two diagrams:

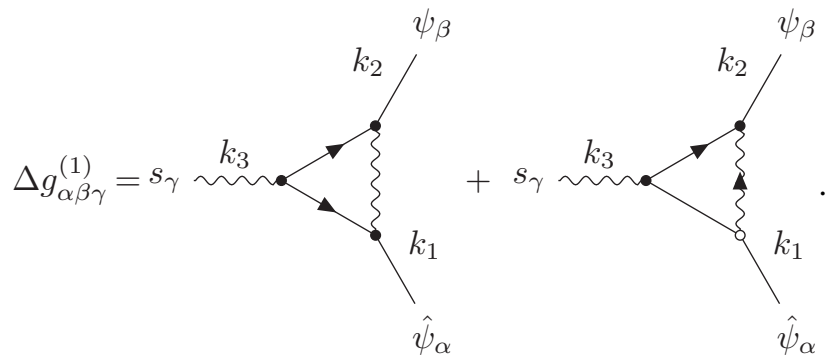

(B1)

After integration over the internal lines, we get expressions of the kind

$$
\Delta g_{\alpha \beta \gamma}^{(1)}=\epsilon_{\alpha_{1} \beta_{1} \gamma} \epsilon_{\alpha_{2} \beta \gamma_{2}} \epsilon_{\alpha \beta_{3} \gamma_{3}} \tau_{\alpha_{1} \beta_{1} \alpha_{2} \gamma_{2} \beta_{3} \gamma_{3}}\left(k_{1}, k_{2}, k_{3}\right),
$$

where we are summing over all repeated indices, and the tensor $\tau$ only depends on the internal indices and on the external momenta. At zero incoming momentum and frequency, because of the symmetry under exchange of the two internal lines of the field $\psi, \tau$ becomes a symmetric tensor. In particular, $\tau(0,0,0)$ is symmetric under exchange of indices $\alpha_{1}$ and $\beta_{1}$. Therefore, the contraction between $\epsilon_{\alpha_{1} \beta_{1} \gamma}$ and $\tau$ is zero. Other possible one-loop corrections may come from both static and dynamic vertices of the kind

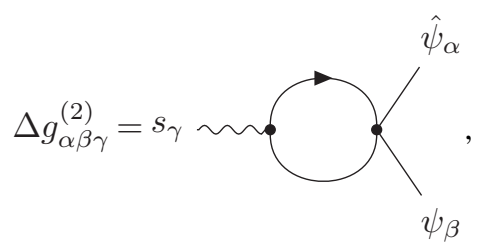

which is of order $g_{0} u_{0}$. Also in this case, at zero incoming momentum and frequency, the correction $\Delta g_{\alpha \beta \gamma}^{(2)}$ is zero by symmetry.
It is possible to extend this reasoning to all orders in perturbation theory; the full perturbative expansion $\Delta g_{\alpha \beta \gamma}$ of vertex (A13) satisfies the following diagrammatic equation,
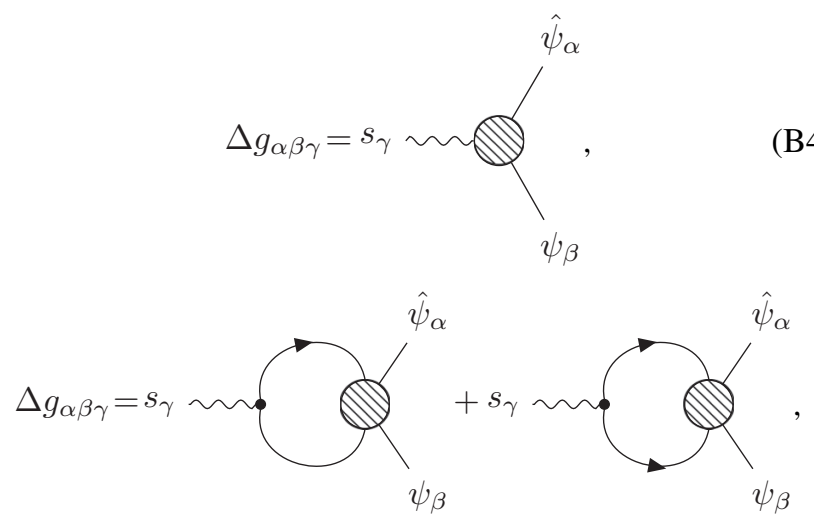

where we explicitly described the possible ways in which the external $s_{\gamma}$ line can attach to the correction diagram. The result is zero for the same symmetry reason as above; the dynamic coupling constant $g_{0}$ therefore has no perturbative corrections at all orders in perturbation theory.

\section{APPENDIX C: WARD IDENTITIES}

The fact that $g_{0}$ has no corrections at all orders in perturbation theory is related to the presence of Ward identities relating response functions (or, equivalently vertex functions [62]) of different order. These identities derive from the fact that the spin is the generator of the rotational symmetry of the order parameter. In the absence of dissipation, the global spin is conserved. In this case, if the system is prepared in an equilibrium state with global polarization $\langle\boldsymbol{\Phi}\rangle$ the effect of an homogeneous field $\mathbf{H}(t)$ coupled to the spin is simply to rotate the polarization, i.e.,

$$
\frac{d\langle\boldsymbol{\Phi}\rangle}{d t}=g_{0} \mathbf{H} \times\langle\boldsymbol{\Psi}\rangle .
$$

Let us now consider a more complex situation where we apply two fields: the first one, $\mathbf{h}(\mathbf{x}, t)$, coupled to the local order parameter, and the second, $\mathbf{H}(t)$, coupled to the spin. The first field will generate a space-dependent local polarization $\langle\psi(\mathbf{x}, t)\rangle$, and the second field will simply homogeneously rotate such local polarizations. If there is dissipation, and the global spin is not conserved, the field $\mathbf{H}(t)$ will also change the value of the global spin, giving a further contribution to the rotation frequency of the $\langle\psi(\mathbf{x}, t)\rangle$. Let us focus on the parts of both fields that are uniquely due to the presence of $\mathbf{H}(t)$; from Eqs. (15) and (16), we get

$$
\begin{gathered}
\frac{d\langle\delta \boldsymbol{\psi}(\mathbf{x}, t)\rangle}{d t}=g_{0}(\mathbf{H}(t)-\delta \boldsymbol{s}(t))\langle\boldsymbol{\psi}(\mathbf{x}, t)\rangle, \\
\frac{d\langle\delta \mathbf{s}(t)\rangle}{d t}=-\eta_{0} \delta \mathbf{s}(t)+\eta_{0} \mathbf{H}(t),
\end{gathered}
$$


where $\delta \boldsymbol{s}$ is the change of spin per unit volume. Integrating both equations, we get

$$
\begin{aligned}
\left\langle\delta \psi_{\alpha}(\mathbf{x}, t)\right\rangle= & g_{0} \epsilon_{\alpha \beta \gamma} \int_{0}^{t} d t^{\prime \prime}\left\langle\psi_{\gamma}\left(\mathbf{x}, t^{\prime \prime}\right)\right\rangle \\
& \times\left[H_{\beta}\left(t^{\prime \prime}\right)-\eta_{0} \int_{0}^{t^{\prime \prime}} d t^{\prime} e^{-\eta_{0}\left(t-t^{\prime}\right)} H_{\beta}\left(t^{\prime}\right)\right] .
\end{aligned}
$$

Both sides in this expression implicitly also depend on $\mathbf{h}(\mathbf{x}, t)$. Let us then derive with respect to this last field and then set it to zero. We get

$$
\begin{aligned}
& \left.\frac{d\left\langle\delta \psi_{\alpha}(\mathbf{x}, t)\right\rangle}{d h_{\delta}\left(\mathbf{x}_{1}, t_{1}\right)}\right|_{h=0}=g_{0} \epsilon_{\alpha \beta \gamma} \int_{0}^{t} d t^{\prime \prime} H_{\beta}\left(t^{\prime \prime}\right) \\
& \quad \times\left[R_{\gamma \delta}^{h}\left(\mathbf{x}, t^{\prime \prime} ; \mathbf{x}_{1}, t_{1}\right)-\eta_{0} \int_{t^{\prime \prime}}^{t} d t^{\prime} e^{-\eta_{0}\left(t^{\prime}-t^{\prime \prime}\right)} R_{\gamma \delta}^{h}\left(\mathbf{x}, t^{\prime} ; \mathbf{x}_{1}, t_{1}\right)\right],
\end{aligned}
$$

where we relabelled integration variables in the second integral for future convenience. Here, $R_{\gamma \delta}^{h}\left(\mathbf{x}, t ; \mathbf{x}_{1}, t_{1}\right)=$ $\partial\left\langle\psi_{\gamma}(\mathbf{x}, t)\right\rangle /\left.\partial h_{\delta}\left(\mathbf{x}_{1}, t_{1}\right)\right|_{h, H=0}$ is the linear response of the order parameter to its conjugate field. Using response theory, the left-hand side of (C5) can also be written as

$$
\left.\frac{d\left\langle\delta \psi_{\alpha}(\mathbf{x}, t)\right\rangle}{d h_{\delta}\left(\mathbf{x}_{1}, t_{1}\right)}\right|_{h=0}=\int_{0}^{t} d t^{\prime \prime} d \mathbf{x}^{\prime \prime} H_{\beta}\left(t^{\prime \prime}\right) R_{\alpha \beta \delta}^{h H}\left(\mathbf{x}, t ; \mathbf{x}_{1}, t_{1} ; \mathbf{x}^{\prime \prime}, t^{\prime \prime}\right),
$$

where now in the right-hand side $R_{\alpha \beta \delta}^{h H}\left(\mathbf{x}, t ; \mathbf{x}_{1}, t_{1} ; \mathbf{x}^{\prime \prime}, t^{\prime \prime}\right)=$ $\partial^{2}\left\langle\psi_{\alpha}(\mathbf{x}, t)\right\rangle /\left.\left(\partial h_{\delta}\left(\mathbf{x}_{1}, t_{1}\right) \partial H_{\beta}\left(\mathbf{x}^{\prime \prime}, t^{\prime \prime}\right)\right)\right|_{h, H=0}$ is the nonlinear quadratic response. Equating the right-hand side of (C5) and (C6), we finally get

$$
\begin{gathered}
\int d \mathbf{x}^{\prime \prime} R_{\alpha \beta \delta}^{h H}\left(\mathbf{x}, t ; \mathbf{x}_{1}, t_{1} ; \mathbf{x}^{\prime \prime}, t^{\prime \prime}\right)=g_{0} \epsilon_{\alpha \beta \gamma}\left[R_{\gamma \delta}^{h}\left(\mathbf{x}, t^{\prime \prime} ; \mathbf{x}_{1}, t_{1}\right)\right. \\
\left.-\eta_{0} \int_{t^{\prime \prime}}^{t} d t^{\prime} e^{-\eta_{0}\left(t^{\prime}-t^{\prime \prime}\right)} R_{\gamma \delta}^{h}\left(\mathbf{x}, t^{\prime} ; \mathbf{x}_{1}, t_{1}\right)\right]
\end{gathered}
$$

with $t_{1}<t^{\prime \prime}<t$. For $\eta_{0}=0$, this relation corresponds to the Ward identity reported for model E in Ref. [43].

\section{APPENDIX D: SHELL INTEGRATION}

To perform an RG transformation, we need to implement two different steps: integration of short wavelength fluctuations and rescaling. To this end, once the coarse-graining factor $b$ is fixed, it is convenient to rewrite the fields as the sum of two distinct components, one fluctuating on short wavelengths $\Lambda / b<k<\Lambda$ and and the other on larger ones
$0<k<\Lambda / b$, i.e.,

$$
\boldsymbol{\psi}(\mathbf{k}, \omega)=\boldsymbol{\psi}^{<}(\mathbf{k}, \omega)+\boldsymbol{\psi}^{>}(\mathbf{k}, \omega) .
$$

At this point, one integrates out explicitly from (A4) the $\boldsymbol{\psi}^{>}$fields, to remain with a measure and a new effective action that only depend on the $\boldsymbol{\psi}^{<}$fields. To perform this integration, one proceeds, again, using perturbation theory. The basic ingredients of this perturbation expansion (free propagators and vertices) are the same as the ones discussed in the previous sections, the difference being that they refer to $\boldsymbol{\psi}^{>}$fields only, while the $\boldsymbol{\psi}^{<}$are kept fixed as external sources. The perturbation series therefore consists in diagrams with external $\psi^{<}$legs and internal loops integrated over $>$propagators. It can be recast in exponential form, as usual, by only retaining one-particle irreducible diagrams. These diagrams, that have external $\boldsymbol{\psi}^{<}$fields attached, will therefore modify the original terms appearing in the action. For example, for the Gaussian part of the action, we get

$$
\begin{aligned}
\mathcal{S}_{0, \psi}^{<}= & \int^{\Lambda / b} \frac{d^{d} k}{(2 \pi)^{d}} \frac{d \omega}{2 \pi} \hat{\boldsymbol{\psi}}^{<}(-\mathbf{k},-\omega)\left[-i \omega+\Gamma_{0}\left(k^{2}+r_{0}^{2}\right)\right. \\
& \left.+\Sigma_{b}(\mathbf{k}, \omega)\right] \boldsymbol{\psi}^{<}(\mathbf{k}, \omega)+\Gamma_{0} \hat{\psi}_{\alpha}(-\mathbf{k},-\omega) \hat{\psi}_{\alpha}(\mathbf{k}, \omega),
\end{aligned}
$$

where $\Sigma_{b}$ has the same expressions as in Eq. (A23) but where integrals are performed only in the shell $\Lambda / b<k<\Lambda$. From this expression, we immediately see that the behavior of the self-energy $\Sigma_{b}$ at small $k$ effectively modifies the coefficient of $k^{2}$. We are then left with a free part of the action similar to the original one, but where integrals run only up to $\Lambda / b$. The second step of the RGT, namely the rescaling of $k, \omega, \psi$, and $\hat{\psi}$, has the purpose of reinstating momentum integrals over their original integration range. At one loop, the renormalization of the field is trivial (i.e., given by its physical dimension) and has therefore not been addressed explicitly in the main text. The result is a new action formally of the same kind as the original one but with a new renormalized kinetic coefficient $\Gamma_{b}$. A similar procedure can be applied also to the free action of the field $s$ and to the interacting part. All the coefficients and coupling constants will get renormalized by the shell integration and rescaling. If we call $\mathcal{P}$ the set of all parameters entering the action, i.e., $\mathcal{P} \equiv\left\{r_{0}, u_{0}, \Gamma_{0}, \eta_{0}, \lambda_{0}, g_{0}\right\}$, an RG transformation will therefore imply

$$
\begin{gathered}
\mathcal{P} \longrightarrow \mathcal{P}_{b}, \\
\mathcal{S}(\mathcal{P}) \longrightarrow \mathcal{S}_{b}=\mathcal{S}\left(\mathcal{P}_{b}\right) .
\end{gathered}
$$

Multiple iterations of the RG transformation therefore define a flow in the space of parameters, i.e., in the space of the statistical models defined by the action (A5).
[1] B. Widom, J. Chem. Phys. 43, 3898 (1965).

[2] L. Kadanoff, Physics (Long Island City, N.Y.) 2, 263 (1966).

[3] B. I. Halperin and P. C. Hohenberg, Phys. Rev. Lett. 19, 700 (1967).

[4] R. A. Ferrell, N. Menyhárd, H. Schmidt, F. Schwabl, and P. Szépfalusy, Phys. Rev. Lett. 18, 891 (1967).
[5] K. G. Wilson, Phys. Rev. B 4, 3174 (1971).

[6] K. G. Wilson, Phys. Rev. D 3, 1818 (1971).

[7] K. G. Wilson and M. E. Fisher, Phys. Rev. Lett. 28, 240 (1972).

[8] K. G. Wilson and J. Kogut, Phys. Rep. 12, 75 (1974).

[9] T. Vicsek and A. Zafeiris, Phys. Rep. 517, 71 (2012). 
[10] M. C. Marchetti, J. F. Joanny, S. Ramaswamy, T. B. Liverpool, J. Prost, M. Rao, and R. A. Simha, Rev. Mod. Phys. 85, 1143 (2013).

[11] A. Cavagna, A. Cimarelli, I. Giardina, G. Parisi, R. Santagati, F. Stefanini, and M. Viale, Proc. Natl. Acad. Sci. USA 107, 11865 (2010).

[12] H.-P. Zhang, A. Be'er, E.-L. Florin, and H. L. Swinney, Proc. Natl. Acad. Sci. USA 107, 13626 (2010).

[13] A. Attanasi, A. Cavagna, L. Del Castello, I. Giardina, S. Melillo, L. Parisi, O. Pohl, B. Rossaro, E. Shen, E. Silvestri et al., PLoS Comput. Biol. 10, e1003697 (2014).

[14] Q.-Y. Tang, Y.-Y. Zhang, J. Wang, W. Wang, and D. R. Chialvo, Phys. Rev. Lett. 118, 088102 (2017).

[15] T. Mora and W. Bialek, J. Stat. Phys. 144, 268 (2011).

[16] B. I. Halperin and P. C. Hohenberg, Phys. Rev. 177, 952 (1969).

[17] S. Bradde and W. Bialek, J. Stat. Phys. 167, 462 (2017).

[18] L. Meshulam, J. L. Gauthier, C. D. Brody, D. W. Tank, and W. Bialek, arXiv:1809.08461; arXiv:1812.11904.

[19] J. Toner, Y. Tu, and S. Ramaswamy, Ann. Phys. 318, 170 (2005).

[20] J. Toner and Y. Tu, Phys. Rev. Lett. 75, 4326 (1995).

[21] J. Toner and Y. Tu, Phys. Rev. E 58, 4828 (1998).

[22] A. Cavagna, D. Conti, C. Creato, L. Del Castello, I. Giardina, T. S. Grigera, S. Melillo, L. Parisi, and M. Viale, Nat. Phys. 13, 914 (2017).

[23] P. C. Hohenberg and B. I. Halperin, Rev. Mod. Phys. 49, 435 (1977).

[24] J. Cardy, Scaling and Renormalization in Statistical Physics (Cambridge University Press, Cambridge, UK, 1996), Vol. 5.

[25] A. Cavagna, L. Del Castello, I. Giardina, T. Grigera, A. Jelic, S. Melillo, T. Mora, L. Parisi, E. Silvestri, M. Viale et al., J. Stat. Phys. 158, 601 (2015).

[26] A. Cavagna, I. Giardina, and T. S. Grigera, Phys. Rep. 728, 1 (2018).

[27] A. Attanasi, A. Cavagna, L. Del Castello, I. Giardina, T. S. Grigera, A. Jelić, S. Melillo, L. Parisi, O. Pohl, E. Shen et al., Nat. Phys. 10, 691 (2014).

[28] A. Attanasi, A. Cavagna, L. Del Castello, I. Giardina, A. Jelic, S. Melillo, L. Parisi, O. Pohl, E. Shen, and M. Viale, J. R. Soc., Interface 12, 20150319 (2015).

[29] V. Zapf, M. Jaime, and C. D. Batista, Rev. Mod. Phys. 86, 563 (2014).

[30] J. Eisenstein and A. MacDonald, Nature (London) 432, 691 (2004).

[31] J. Klaers, J. Schmitt, F. Vewinger, and M. Weitz, Nature (London) 468, 545 (2010).

[32] A. Cavagna, L. Di Carlo, I. Giardina, L. Grandinetti, T. S. Grigera, and G. Pisegna, Phys. Rev. Lett. 123, 268001 (2019).

[33] F. Ginelli, Eur. Phys. J.: Spec. Top. 225, 2099 (2016).

[34] T. Vicsek, A. Czirók, E. Ben-Jacob, I. Cohen, and O. Shochet, Phys. Rev. Lett. 75, 1226 (1995).

[35] N. D. Mermin and H. Wagner, Phys. Rev. Lett. 17, 1133 (1966).

[36] A. Attanasi, A. Cavagna, L. Del Castello, I. Giardina, S. Melillo, L. Parisi, O. Pohl, B. Rossaro, E. Shen, E. Silvestri et al., Phys. Rev. Lett. 113, 238102 (2014).

[37] Y. Tu, J. Toner, and M. Ulm, Phys. Rev. Lett. 80, 4819 (1998).
[38] A. Cavagna and I. Giardina, Annu. Rev. Condens. Matter Phys. 5, 183 (2014).

[39] A. Cavagna, I. Giardina, T. S. Grigera, A. Jelic, D. Levine, S. Ramaswamy, and M. Viale, Phys. Rev. Lett. 114, 218101 (2015).

[40] N. Goldenfeld, Lectures on Phase Transitions and the Renormalization Group (Perseus Books, Reading, MA, 1992).

[41] J. J. Binney, N. Dowrick, A. Fisher, and M. Newman, The Theory of Critical Phenomena: An Introduction to the Renormalization Group (Oxford University Press, Oxford, UK, 1992).

[42] U. C. Täuber, Critical Dynamics: A Field Theory Approach to Equilibrium and Non-equilibrium Scaling Behavior (Cambridge University Press, Cambridge, UK, 2014).

[43] B. Halperin, P. Hohenberg, and E. Siggia, Phys. Rev. B 13, 1299 (1976).

[44] C. De Dominicis and L. Peliti, Phys. Rev. B 18, 353 (1978).

[45] C. Lanczos, Linear Differential Operators, Classics in Applied Mathematics, Vol. 393 (SIAM, London, UK, 1961).

[46] G. Parisi, Statistical Field Theory, Frontiers in Physics (Addison-Wesley, Redwood City, CA, 1988).

[47] D. J. Amit and V. Martin-Mayor, Field Theory, the Renormalization Group, and Critical Phenomena: Graphs to Computers, 3rd ed. (World Scientific, Singapore, 2005).

[48] B. Halperin, P. Hohenberg, and S.-k. Ma, Phys. Rev. Lett. 29, 1548 (1972).

[49] P. C. Martin, E. D. Siggia, and H. A. Rose, Phys. Rev. A 8, 423 (1973).

[50] M. Le Bellac, Quantum and Statistical Field Theory (Clarendon Press, Oxford, UK, 1991).

[51] C. Itzykson and J.-M. Drouffe, Statistical Field Theory. Volume 1: From Brownian Motion to Renormalization and Lattice Gauge Theory, 1st ed., Cambridge Monographs on Mathematical Physics, Vol. 1 (Cambridge University Press, Cambridge, UK, 1989).

[52] J. Zinn-Justin, Quantum Field Theory and Critical Phenomena, 4th ed., International Series of Monographs on Physics Vol. 113 (Oxford University Press, New York, 2002).

[53] E. Frey, Physica A (Amsterdam, Neth.) 221, 52 (1995).

[54] E. Frey and F. Schwabl, Phys. Rev. B 42, 8261 (1990).

[55] M. P. Allen and D. J. Tildesley, Computer Simulation of Liquids (Clarendon Press, Oxford, UK, 1987).

[56] M. Allen, Mol. Phys. 40, 1073 (1980).

[57] W. C. Swope, H. C. Andersen, P. H. Berens, and K. R. Wilson, J. Chem. Phys. 76, 637 (1982).

[58] A. Cavagna, D. Conti, I. Giardina, T. S. Grigera, S. Melillo, and M. Viale, Phys. Biol. 13, 065001 (2016).

[59] M. N. Barber, in Phase Transitions and Critical Phenomena, edited by C. Domb and J. L. Lebowitz (Academic Press, London, 1983), Vol. 8.

[60] K. Binder, Ferroelectrics 73, 43 (1987).

[61] C. De Dominicis and I. Giardina, Random Fields and Spin Glasses: A Field Theory Approach (Cambridge University Press, Cambridge, UK, 2006).

[62] L. H. Ryder, Quantum Field Theory (Cambridge University Press, Cambridge, UK, 1996). 